\title{
Asymptotic properties of maximum likelihood estimator for the growth rate of a stable CIR process based on continuous time observations
}

\author{
Mátyás Barczy ${ }^{* \diamond, ~ M o h a m e d ~ B e n ~ A l a y a * *, ~}$ \\ Ahmed Kebaier ${ }^{* * *}$ And Gyula PAP****
}

* MTA-SZTE Analysis and Stochastics Research Group, Bolyai Institute, University of Szeged, Aradi vértanúk tere 1, H-6720 Szeged, Hungary.

** Laboratoire De Mathématiques Raphaël Salem, UMR 6085, Université De Rouen, Avenue de L’Université Technopôle du Madrillet, 76801 Saint-Etienne-Du-Rouvray, France.

*** Université Paris 13, Sorbonne Paris Cité, LAGA, CNRS (UMR 7539), Villetaneuse, France.

**** Bolyai Institute, University of Szeged, Aradi vértanúk tere 1, H-6720 Szeged, Hungary.

e-mails: barczy@math.u-szeged.hu (M. Barczy), mohamed.ben-alaya@univ-rouen.fr (M. Ben Alaya), kebaier@math.univ-paris13.fr (A. Kebaier), papgy@math.u-szeged.hu (G. Pap).

$\diamond$ Corresponding author.

\|\|

\begin{abstract}
We consider a stable Cox-Ingersoll-Ross process driven by a standard Wiener process and a spectrally positive strictly stable Lévy process, and we study asymptotic properties of the maximum likelihood estimator (MLE) for its growth rate based on continuous time observations. We distinguish three cases: subcritical, critical and supercritical. In all cases we prove strong consistency of the MLE in question, in the subcritical case asymptotic normality, and in the supercritical case asymptotic mixed normality are shown as well. In the critical case the description of the asymptotic behavior of the MLE in question remains open.
\end{abstract}

\section{Introduction}

We consider a jump-type Cox-Ingersoll-Ross (CIR) process driven by a standard Wiener process and a spectrally positive strictly $\alpha$-stable Lévy process given by the SDE

$$
\mathrm{d} Y_{t}=\left(a-b Y_{t}\right) \mathrm{d} t+\sigma \sqrt{Y_{t}} \mathrm{~d} W_{t}+\delta \sqrt[\alpha]{Y_{t-}} \mathrm{d} L_{t}, \quad t \in[0, \infty),
$$

2010 Mathematics Subject Classifications: 60H10, 91G70, 60F05, 62F12.

Key words and phrases: stable Cox-Ingersoll-Ross process, maximum likelihood estimator.

This research is supported by Laboratory of Excellence MME-DII, Grant no. ANR11-LBX-0023-01 (http://labex-mme-dii.u-cergy.fr/). Mátyás Barczy was supported between September 2016 and January 2017 by the "Magyar Állami Eötvös Ösztöndíj 2016" Grant no. 75141 funded by the Tempus Public Foundation, and from September 2017 by the János Bolyai Research Scholarship of the Hungarian Academy of Sciences. Ahmed Kebaier benefited from the support of the chair Risques Financiers, Fondation du Risque. 
with an almost surely non-negative initial value $Y_{0}$, where $a \in[0, \infty), b \in \mathbb{R}, \sigma \in[0, \infty), \delta \in(0, \infty)$, $\alpha \in(1,2), \quad\left(W_{t}\right)_{t \in[0, \infty)}$ is a 1-dimensional standard Wiener process, and $\left(L_{t}\right)_{t \in[0, \infty)}$ is a spectrally positive $\alpha$-stable Lévy process such that the characteristic function of $L_{1}$ takes the form

$$
\mathbb{E}\left(\mathrm{e}^{\mathrm{i} \theta L_{1}}\right)=\exp \left\{\int_{0}^{\infty}\left(\mathrm{e}^{\mathrm{i} \theta z}-1-\mathrm{i} \theta z\right) C_{\alpha} z^{-1-\alpha} \mathrm{d} z\right\}, \quad \theta \in \mathbb{R},
$$

where $C_{\alpha}:=(\alpha \Gamma(-\alpha))^{-1}$ and $\Gamma$ denotes the Gamma function. In fact, $\left(L_{t}\right)_{t \in[0, \infty)}$ is a strictly $\alpha$-stable Lévy process, see, e.g., Sato [35, part (vi) of Theorem 14.7]. We suppose that $Y_{0},\left(W_{t}\right)_{t \in[0, \infty)}$ and $\left(L_{t}\right)_{t \in[0, \infty)}$ are independent. Under the given conditions together with $\mathbb{E}\left(Y_{0}\right)<\infty$, there is a (pathwise) unique strong solution of the SDE (1.1) with $\mathbb{P}\left(Y_{t} \in[0, \infty)\right.$ for all $\left.t \in[0, \infty)\right)=1$. As a matter of fact, the SDE (1.1) is a special case of the SDE (1.8) in Fu and Li [15] (with the special choice $z_{1} \equiv 0$ ), for which the existence of a pathwise unique non-negative strong solution has been proved (see Fu and Li [15, Corollary 6.3]). Eventually, the process $\left(Y_{t}\right)_{t \in[0, \infty)}$ given by the SDE (1.1) is a continuous state and continuous time branching process with immigration (CBI process), see (ii) of Proposition [2.1. We call $Y$ an $\alpha$-stable CIR process (or Alpha-CIR process), which is a generalization of the usual CIR process (given by the SDE (1.1) formally with $\delta=0$ ).

Stable CIR processes become more and more popular in stochastic modelling, and it is an interesting class of CBI processes on its own right as well. Carr and Wu [9, equation (31)] considered a stochastic process admitting an infinitesimal generator which coincides with the corresponding one of an $\alpha$-stable CIR process with $\sigma=0$, see (iv) of Proposition 2.1 ,

Li and Ma 26] proved exponential ergodicity for the process $\left(Y_{t}\right)_{t \in[0, \infty)}$ provided that $a \in(0, \infty)$ and $b \in(0, \infty)$, for more details, see (ii) of Theorem 2.5. Li and Ma [26] also described the asymptotic behavior of the conditional least squares estimator (LSE) and weighted conditional LSE of the drift parameters $(a, b)$ of an $\alpha$-stable CIR process given by the SDE (1.1) with $\sigma=0$, based on (discretely observed) low frequency observations in the subcritical case (i.e., when $b \in(0, \infty))$. In the region $\alpha \in\left(1, \frac{1+\sqrt{5}}{2}\right), \mathrm{Li}$ and Ma [26] showed that the normalizing factor for the LSE of $(a, b)$ is $n^{(\alpha-1) / \alpha^{2}}$, which is quite different from the $\sqrt{n}$-normalization being quite usual for subcritical models. On the top of it all, $\mathrm{Li}$ and $\mathrm{Ma}$ [26] also proved that the corresponding normalizing factor for the weighted LSE of $(a, b)$ is $n^{(\alpha-1) / \alpha}$ (being different from the one for the (usual) LSE) in the whole region $\alpha \in(1,2)$.

Jiao et al. [19] investigated several properties of $\alpha$-stable CIR processes such as integral representations, branching property in the pathwise sense, necessary and sufficient conditions for strictly positiveness and they made an analysis of the jumps of the process. Further, they used $\alpha$-stable CIR processes for interest rate modelling and pricing by pointing out that these processes can describe some recent phenomena on sovereign bond market such as large fluctuations at a local extent together with the usual small oscillations, for more details, see the Introduction of Jiao et al. [19]. Very recently, Jiao et al. 20] have proposed concrete examples of applications and investigated a factor model for electricity prices, where $\alpha$-stable CIR processes may appear as factors of the model in question.

Peng [33] introduced and studied a so-called $\alpha$-stable CIR process with restart, by which one means that the process in question behaves as an $\alpha$-stable CIR process given by the SDE (1.1) with $\sigma=0$, it is killed at the boundary 0 of $[0, \infty)$, and according to an exponential clock it jumps to a new point in $[0, \infty)$ according to a given probability distribution on $[0, \infty)$. As it was pointed out in Peng [33], restart phenomenon appears in internet congestion as well: whenever a web page takes too 
much time to appear, it is useful to press the reload button and then usually the web page appears immediately.

Yang [38] studied $\alpha$-stable CIR processes with small $\alpha$-stable noises given by the SDE

$$
\mathrm{d} Y_{t}^{\varepsilon}=\left(a-b Y_{t}^{\varepsilon}\right) \mathrm{d} t+\delta \varepsilon \sqrt[q]{Y_{t-}^{\varepsilon}} \mathrm{d} L_{t}, \quad t \in[0, \infty)
$$

with a non-negative deterministic initial value $Y_{0}^{\varepsilon}=y_{0} \in[0, \infty)$, where $q \in\left(0, \frac{1}{1-1 / \alpha}\right)$ and $\varepsilon \in(0, \infty)$. The asymptotic behavior of an approximate maximum likelihood estimator (MLE) of $(a, b, \delta)$ has been described based on discrete time observations at $n$ regularly spaced time points $\frac{k}{n}, k \in\{1, \ldots, n\}$, on a fixed time interval $[0,1]$. Tending $\varepsilon$ to 0 and $n \rightarrow \infty$ at a given rate, for some restricted parameter set, Yang [38, Theorem 2.4] proved asymptotic normality of the approximate MLE in question. In some sense it is surprising, since this restricted parameter set contains parameters belonging to critical $(b=0)$ and supercritical $(b \in(-\infty, 0))$ models as well both with normal limit distributions, and for critical models, the limit distribution, in general, is not even mixed normal.

Ma and Yang [31] investigated asymptotic behavior of the LSE of a for the model (1.3) (all the other parameters are supposed to be known) based on discrete time observations as in Yang [38] described above. They described the asymptotic behavior of the LSE in question and derived large and moderate deviation inequalities for it as well, see Ma and Yang [31, Theorems 2.1, 2.3-2.5].

In this paper, supposing that $a \in[0, \infty), \sigma, \delta \in(0, \infty)$ and $\alpha \in(1,2)$ are known, we study the asymptotic properties of the MLE of $b \in \mathbb{R}$ based on continuous time observations $\left(Y_{t}\right)_{t \in[0, T]}$ with $T \in(0, \infty)$, starting the process $Y$ from some known non-random initial value $y_{0} \in[0, \infty)$.

The paper is organized as follows. Section 2 is devoted to some preliminaries. First, we recall some useful properties of the stable CIR process $\left(Y_{t}\right)_{t \in[0, \infty)}$ given by the SDE (1.1) such as the existence of a non-negative pathwise unique strong solution, the forms of the Laplace transform and the infinitesimal generator or conditions on the strictly positiveness of the process or the integrated process, see Proposition 2.1. We derive a so-called Grigelionis form of the semimartingale $\left(Y_{t}\right)_{t \in[0, \infty)}$, see Proposition 2.2. Based on the asymptotic behavior of the expectation of $Y_{t}$ as $t \rightarrow \infty$, we distinguish subcritical, critical or supercritical cases according to $b \in(0, \infty), b=0$ or $b \in(-\infty, 0)$, see Proposition 2.3 and Definition 2.4. In Proposition 2.3 it also turns out that the parameter $b$ can be interpreted as a growth rate of the model. We recall a result about the existence of a unique stationary distribution for the process $\left(Y_{t}\right)_{t \in[0, \infty)}$ in the subcritical and critical cases, and about its exponential ergodicity in the subcritical case, due to Li [25], Li and Ma [26] and Jin et al. [21], see Theorem 2.5. We call the attention that there exists a unique stationary distribution for $\left(Y_{t}\right)_{t \in[0, \infty)}$ in the critical case as well. Remark 2.6 is devoted to give an alternative proof for the weak convergence of $Y_{t}$ as $t \rightarrow \infty$ in Theorem 2.5 in case of $\sigma \in(0, \infty)$, giving more insight as well. In Remark 2.7, we give a statistic for $\sigma^{2}$ using continuous time observations $\left(Y_{t}\right)_{t \in[0, T]}$ with an arbitrary $T \in(0, \infty)$, and due to this result we do not consider the estimation of the parameter $\sigma$, it is supposed to be known. In Section 3, we derive a formula for the joint Laplace transform of $Y_{t}$ and $\int_{0}^{t} Y_{s} \mathrm{~d} s$, where $t \in[0, \infty)$, using Theorem 4.10 in Keller-Ressel [22], see Theorem 3.1. We note that this form of the joint Laplace transform in question is a consequence of Theorem 5.3 in Filipović [13], a special case of Proposition 3.3 in Jiao et al. [19] as well, and it is used for describing the asymptotic behavior of the MLE of $b$ in question in the critical and supercritical cases. Section 4 is devoted to prove the existence and uniqueness of the MLE of $b$ (provided that $\sigma \in(0, \infty)$ ) deriving an explicit formula for it as 
well, see Proposition 4.2, In Remark 4.3, under the additional assumption $a \in\left[\frac{\sigma^{2}}{2}, \infty\right)$, we prove that $L_{t}$ is a measurable function of $\left(Y_{u}\right)_{u \in[0, T]}$ for all $t \in[0, T]$ with any $T \in(0, \infty)$. In Section 5. provided that $a \in(0, \infty)$, we prove strong consistency and asymptotic normality of the MLE of $b$ in the subcritical case, see Theorem 5.1. The asymptotic normality in question holds with a usual square root normalization $(\sqrt{T})$, but as usual, the asymptotic variance depends on the unknown parameter $b$, as well. To get around this problem, we also replace the normalization $\sqrt{T}$ by a random one $\frac{1}{\sigma}\left(\int_{0}^{t} Y_{s} \mathrm{~d} s\right)^{1 / 2}$ (depending only on the observation, but not on the parameter $b$ ) with the advantage that the MLE of $b$ with this random scaling is asymptotically standard normal, so one can give asymptotic confidence intervals for the unknown parameter $b$, which is desirable for practical purposes. Section 6 is devoted to prove the strong consistency of the MLE of $b$ in the critical case, provided that $a \in(0, \infty)$, (see Theorem 6.2) using the limit behavior of the unique locally bounded solution of the differential equation (3.1) at infinity described in Proposition 6.1, We call the attention to the fact that for the $\alpha$-stable CIR process $\left(Y_{t}\right)_{t \in[0, \infty)}$, the critical case $(b=0)$ is somewhat special (compared to the original CIR process with $b=0$ ), since there still exists a unique stationary distribution for $\left(Y_{t}\right)_{t \in[0, \infty)}$, however its expectation is infinite unless $a=0$ (see Theorem 2.5), and surprisingly, we can prove strong consistency of the MLE in question not only weak consistency usually proved for critical models. In the critical case the description of the asymptotic behavior of the MLE remains open. In Section [7, for the supercritical case, provided that $a \in(0, \infty)$, we prove that the MLE of $b$ is strongly consistent and asymptotically mixed normal with the deterministic scaling $\mathrm{e}^{-b T / 2}$, and it is asymptotically standard normal with the random scaling $\frac{1}{\sigma}\left(\int_{0}^{t} Y_{s} \mathrm{~d} s\right)^{1 / 2}$, see Theorem 7.4. We point out that the limit mixed normal law in question is characterized in a somewhat complicated way, namely in its description a positive random variable $V$ comes into play of which the Laplace transform contains a function related to the branching mechanism of the CBI process $\left(Y_{t}\right)_{t \in[0, \infty)}$, see Theorem 7.1. We give two proofs for the derivation of the Laplace transform of $V$, and the second one is heavily based on the general theory of CBI processes, for which we will refer to Li [25]. We close the paper with three Appendices, where we recall certain sufficient conditions for the absolute continuity of probability measures induced by semimartingales together with a representation of the Radon-Nikodym derivative (Appendix $(\mathrm{A})$, some limit theorems for continuous local martingales (Appendix B) and in case of a $\frac{3}{2}$-stable CIR process we present some explicit formulae for the Laplace transform of the unique stationary distribution in the subcritical and critical cases, of $Y_{t}, t \in[0, \infty)$, in all the cases of $b \in \mathbb{R}$, and of $V$ in the supercritical case, respectively (Appendix $\mathbb{C}$ ).

Finally, we summarize the novelties of the paper. According to our knowledge, maximum likelihood estimation based on continuous time observations has never been studied before for the $\alpha$-stable CIR process $\left(Y_{t}\right)_{t \in[0, \infty)}$, and since these processes become more and more popular in financial mathematics and market models for electricity prices, the problem of estimating its parameters is an important question as well. Further, in the critical case, somewhat surprisingly, we can prove strong consistency of the MLE of $b$, which can be considered as a new phenomenon, since for other critical financial models, such as for the usual CIR process or for the Heston process, only weak consistency is proved in the critical case, see Overbeck [32, Theorem 2, parts (iii) and (iv)] and Barczy and Pap [6, Remark 4.4], respectively. 


\section{Preliminaries}

Let $\mathbb{N}, \mathbb{Z}_{+}, \mathbb{R}, \mathbb{R}_{+}, \mathbb{R}_{++}, \mathbb{R}_{-}, \mathbb{R}_{--}$and $\mathbb{C}$ denote the sets of positive integers, non-negative integers, real numbers, non-negative real numbers, positive real numbers, non-positive real numbers, negative real numbers and complex numbers, respectively. For $x, y \in \mathbb{R}$, we will use the notations $x \wedge y:=\min (x, y)$ and $x \vee y:=\max (x, y)$. The integer part of a real number $x \in \mathbb{R}$ is denoted by $\lfloor x\rfloor$. By $\|x\|$ and $\|A\|$, we denote the Euclidean norm of a vector $x \in \mathbb{R}^{d}$ and the induced matrix norm of a matrix $A \in \mathbb{R}^{d \times d}$, respectively. By $\mathcal{B}\left(\mathbb{R}_{+}\right)$, we denote the Borel $\sigma$-algebra on $\mathbb{R}_{+}$. We will denote the convergence in probability, in distribution and almost surely, and almost sure equality by $\stackrel{\mathbb{P}}{\longrightarrow}, \stackrel{\mathcal{D}}{\longrightarrow}, \stackrel{\text { a.s. }}{\longrightarrow}$ and $\stackrel{\text { a.s. }}{=}$, respectively. By $C_{c}^{2}\left(\mathbb{R}_{+}, \mathbb{R}\right)$ and $C_{c}^{\infty}\left(\mathbb{R}_{+}, \mathbb{R}\right)$, we denote the set of twice continuously differentiable real-valued functions on $\mathbb{R}_{+}$with compact support and the set of infinitely differentiable real-valued functions on $\mathbb{R}_{+}$with compact support, respectively.

Let $\left(\Omega, \mathcal{F},\left(\mathcal{F}_{t}\right)_{t \in \mathbb{R}_{+}}, \mathbb{P}\right)$ be a filtered probability space satisfying the usual conditions, i.e., $(\Omega, \mathcal{F}, \mathbb{P})$ is complete, the filtration $\left(\mathcal{F}_{t}\right)_{t \in \mathbb{R}_{+}}$is right-continuous, $\mathcal{F}_{0}$ contains all the $\mathbb{P}$-null sets in $\mathcal{F}$, and $\mathcal{F}=\sigma\left(\bigcup_{t \in \mathbb{R}_{+}} \mathcal{F}_{t}\right)$. Let $\left(W_{t}\right)_{t \in \mathbb{R}_{+}}$be a standard Wiener process with respect to the filtration $\left(\mathcal{F}_{t}\right)_{t \in \mathbb{R}_{+}}$, and $\left(L_{t}\right)_{t \in \mathbb{R}_{+}}$be a spectrally positive strictly $\alpha$-stable Lévy process with respect to the filtration $\left(\mathcal{F}_{t}\right)_{t \in \mathbb{R}_{+}}$such that the characteristic function of $L_{1}$ is given by (1.2). We assume that $W$ and $L$ are independent. Recall that the Lévy-Itô's representation of $L$ takes the form

$$
L_{t}=\int_{(0, t]} \int_{(0, \infty)} z \widetilde{\mu}^{L}(\mathrm{~d} s, \mathrm{~d} z)=\gamma t+\int_{(0, t]} \int_{(0,1]} z \widetilde{\mu}^{L}(\mathrm{~d} s, \mathrm{~d} z)+\int_{(0, t]} \int_{(1, \infty)} z \mu^{L}(\mathrm{~d} s, \mathrm{~d} z)
$$

for $t \in \mathbb{R}_{+}$, where $\mu^{L}(\mathrm{~d} s, \mathrm{~d} z):=\sum_{u \in \mathbb{R}_{+}} \mathbb{1}_{\left\{\Delta L_{u} \neq 0\right\}} \varepsilon_{\left(u, \Delta L_{u}\right)}(\mathrm{d} s, \mathrm{~d} z)$ is the integer-valued Poisson random measure on $\mathbb{R}_{++}^{2}$ associated with the jumps $\Delta L_{u}:=L_{u}-L_{u-}, \quad u \in \mathbb{R}_{++}, \quad \Delta L_{0}:=0$, of the process $L$, and $\varepsilon_{(u, x)}$ denotes the Dirac measure at the point $(u, x) \in \mathbb{R}_{+}^{2}, \widetilde{\mu}^{L}(\mathrm{~d} s, \mathrm{~d} z):=$ $\mu^{L}(\mathrm{~d} s, \mathrm{~d} z)-\mathrm{d} s m(\mathrm{~d} z)$, where $m(\mathrm{~d} z):=C_{\alpha} z^{-1-\alpha} \mathbb{1}_{(0, \infty)}(z) \mathrm{d} z, \quad$ and $\gamma:=-\int_{(1, \infty)} z \mathrm{~d} s m(\mathrm{~d} z)=$ $-C_{\alpha} \int_{1}^{\infty} z^{-\alpha} \mathrm{d} z=\frac{C_{\alpha}}{1-\alpha}$. The measure $m$ is nothing else but the Lévy measure of $L$. We also note that $\left(L_{t}\right)_{t \in \mathbb{R}_{+}}$is a martingale and consequently $\mathbb{E}\left(L_{t}\right)=0, t \in \mathbb{R}_{+}$.

The next proposition is about the existence and uniqueness of a strong solution of the SDE (1.1) stating also that $Y$ is a CBI process with explicitly given branching and immigration mechanisms and we also collect some other useful properties of $Y$ based on Dawson and Li [10], Fu and Li [15], Li 25] and Jiao et al. [19].

2.1 Proposition. Let $\eta_{0}$ be a random variable independent of $\left(W_{t}\right)_{t \in \mathbb{R}_{+}}$and $\left(L_{t}\right)_{t \in \mathbb{R}_{+}}$satisfying $\mathbb{P}\left(\eta_{0} \in \mathbb{R}_{+}\right)=1$ and $\mathbb{E}\left(\eta_{0}\right)<\infty$. Let $a \in \mathbb{R}_{+}, \quad b \in \mathbb{R}, \quad \sigma \in \mathbb{R}_{+}, \quad \delta \in \mathbb{R}_{++}$, and $\alpha \in(1,2)$. Then the following statements hold.

(i) There exists a pathwise unique strong solution $\left(Y_{t}\right)_{t \in \mathbb{R}_{+}}$of the $S D E$ (1.1) such that $\mathbb{P}\left(Y_{0}=\right.$ $\left.\eta_{0}\right)=1$ and $\mathbb{P}\left(Y_{t} \in \mathbb{R}_{+}\right.$for all $\left.t \in \mathbb{R}_{+}\right)=1$.

(ii) The process $\left(Y_{t}\right)_{t \in \mathbb{R}_{+}}$is a CBI process having branching mechanism

$$
R(z)=\frac{\sigma^{2}}{2} z^{2}+\frac{\delta^{\alpha}}{\alpha} z^{\alpha}+b z, \quad z \in \mathbb{R}_{+},
$$

and immigration mechanism

$$
F(z)=a z, \quad z \in \mathbb{R}_{+}
$$


(iii) For all $t \in \mathbb{R}_{+}$and $y_{0} \in \mathbb{R}_{+}$, the Laplace transform of $Y_{t}$ takes the form

$$
\mathbb{E}\left(\mathrm{e}^{-\lambda Y_{t}} \mid Y_{0}=y_{0}\right)=\exp \left\{-y_{0} v_{t}(\lambda)-\int_{0}^{t} F\left(v_{s}(\lambda)\right) \mathrm{d} s\right\}
$$

for all $\lambda \in \mathbb{R}_{+}$, where $\mathbb{R}_{+} \ni t \mapsto v_{t}(\lambda) \in \mathbb{R}_{+}$is the unique locally bounded solution to

$$
\frac{\partial}{\partial t} v_{t}(\lambda)=-R\left(v_{t}(\lambda)\right), \quad v_{0}(\lambda)=\lambda
$$

If $t \in \mathbb{R}_{+}, y_{0} \in \mathbb{R}_{+}$and $\lambda \in \mathbb{R}_{++} \backslash\left\{\theta_{0}\right\}$ with $\theta_{0}:=\inf \left\{z \in \mathbb{R}_{++}: R(z) \in \mathbb{R}_{+}\right\} \in \mathbb{R}_{+}$, then we have

$$
\mathbb{E}\left(\mathrm{e}^{-\lambda Y_{t}} \mid Y_{0}=y_{0}\right)=\exp \left\{-y_{0} v_{t}(\lambda)+\int_{\lambda}^{v_{t}(\lambda)} \frac{F(z)}{R(z)} \mathrm{d} z\right\} .
$$

Especially, (2.4) holds for all $\lambda \in \mathbb{R}_{++}$whenever $b \in \mathbb{R}_{+}$.

(iv) The infinitesimal generator of $Y$ takes the form

$$
(\mathcal{A} f)(y)=(a-b y) f^{\prime}(y)+\frac{\sigma^{2}}{2} y f^{\prime \prime}(y)+\delta^{\alpha} y \int_{0}^{\infty}\left(f(y+z)-f(y)-z f^{\prime}(y)\right) C_{\alpha} z^{-1-\alpha} \mathrm{d} z
$$

where $y \in \mathbb{R}_{+}, \quad f \in C_{c}^{2}\left(\mathbb{R}_{+}, \mathbb{R}\right)$, and $f^{\prime}$ and $f^{\prime \prime}$ denote the first and second order partial derivatives of $f$.

(v) If, in addition, $\mathbb{P}\left(\eta_{0} \in \mathbb{R}_{++}\right)=1$ or $a \in \mathbb{R}_{++}$, then $\mathbb{P}\left(\int_{0}^{t} Y_{s} \mathrm{~d} s \in \mathbb{R}_{++}\right)=1$ for all $t \in \mathbb{R}_{++}$.

(vi) If, in addition, $\sigma \in \mathbb{R}_{++}$and $a \geqslant \frac{\sigma^{2}}{2}$, then $\mathbb{P}\left(Y_{t} \in \mathbb{R}_{++}\right.$for all $\left.t \in \mathbb{R}_{++}\right)=1$.

(vii) If, in addition, $\mathbb{P}\left(\eta_{0} \in \mathbb{R}_{++}\right)=1, a=0$ and $b \in \mathbb{R}_{+}$, then $\mathbb{P}\left(\tau_{0}<\infty\right)=1$, where $\tau_{0}:=\inf \left\{s \in \mathbb{R}_{+}: Y_{s}=0\right\}$, and $\mathbb{P}\left(Y_{t}=0\right.$ for all $\left.t \geqslant \tau_{0}\right)=1$.

Proof. For the existence of a pathwise unique non-negative strong solution satisfying $\mathbb{P}\left(Y_{0}=\eta_{0}\right)=1$ and $\mathbb{P}\left(Y_{t} \in \mathbb{R}_{+}\right.$for all $\left.t \in \mathbb{R}_{+}\right)=1$, see Fu and Li [15, Corollary 6.3], which yields (i).

Further, Theorem 6.2 in Dawson and Li [10] together with

$$
\int_{0}^{\infty}\left(z \wedge z^{2}\right) C_{\alpha} z^{-1-\alpha} \mathrm{d} z=C_{\alpha} \int_{0}^{1} z^{1-\alpha} \mathrm{d} z+C_{\alpha} \int_{1}^{\infty} z^{-\alpha} \mathrm{d} z=C_{\alpha}\left(\frac{1}{2-\alpha}+\frac{1}{\alpha-1}\right)<\infty
$$

and

$$
\int_{0}^{\infty}\left(\mathrm{e}^{-z x}-1+z x\right) C_{\alpha} x^{-1-\alpha} \mathrm{d} x=\frac{1}{\alpha} \frac{\alpha(\alpha-1)}{\Gamma(2-\alpha)} \int_{0}^{\infty}\left(\mathrm{e}^{-z x}-1+z x\right) x^{-1-\alpha} \mathrm{d} x=\frac{z^{\alpha}}{\alpha}
$$

for $z \in \mathbb{R}_{+}$(see, e.g., Li [25, Example 1.9]) imply that $Y$ is a CBI process having branching and immigration mechanisms given in (ii).

For formula (2.2) and, in case of $b \in \mathbb{R}_{+}$, formula (2.4) see Li [25, formula (3.29) and page 67]. Next we check that

$$
-\int_{0}^{t} F\left(v_{s}(\lambda)\right) \mathrm{d} s=\int_{\lambda}^{v_{t}(\lambda)} \frac{F(z)}{R(z)} \mathrm{d} z
$$


for all $t \in \mathbb{R}_{+}$and $\lambda \in \mathbb{R}_{++} \backslash\left\{\theta_{0}\right\}$. It is enough to verify that the continuously differentiable function $(0, t) \ni s \mapsto v_{s}(\lambda)$ is strictly monotone for all $\lambda \in \mathbb{R}_{++} \backslash\left\{\theta_{0}\right\}$, since then, by the substitution $z=v_{s}(\lambda)$, we obtain

$$
-\int_{0}^{t} F\left(v_{s}(\lambda)\right) \mathrm{d} s=-\int_{\lambda}^{v_{t}(\lambda)} \frac{F(z)}{\frac{\partial}{\partial s} v_{s}\left(\widetilde{v}_{z}(\lambda)\right)} \mathrm{d} z=\int_{\lambda}^{v_{t}(\lambda)} \frac{F(z)}{R\left(v_{s}\left(\widetilde{v}_{z}(\lambda)\right)\right)} \mathrm{d} z
$$

and hence (2.7), where $\left(v_{0}(\lambda) \wedge v_{t}(\lambda), v_{0}(\lambda) \vee v_{t}(\lambda)\right) \ni z \mapsto \widetilde{v}_{z}(\lambda)$ denotes the inverse of $(0, t) \ni$ $s \mapsto v_{s}(\lambda)$. By Li [25, Proposition 3.1], the function $\mathbb{R}_{+} \ni \lambda \mapsto v_{s}(\lambda) \in \mathbb{R}_{+}$is strictly increasing for all $s \in \mathbb{R}_{+}$. We have $v_{s}\left(\theta_{0}\right)=\theta_{0}$ for all $s \in \mathbb{R}_{+}$, since $R\left(\theta_{0}\right)=0$ yields that this constant function is the unique locally bounded solution to the differential equation (2.3) with initial value $\theta_{0}$. If $b \in \mathbb{R}_{+}$, then $\theta_{0}=0$, thus $\lambda \in \mathbb{R}_{++}$implies $v_{s}(\lambda)>v_{s}(0)=0$ for all $s \in \mathbb{R}_{+}$. In this case, using the differential equation (2.3) and the inequality $R(z)>0$ for all $z \in \mathbb{R}_{++}$, we obtain $\frac{\partial}{\partial s} v_{s}(\lambda)=-R\left(v_{s}(\lambda)\right)<0$ for all $s \in \mathbb{R}_{+}$, hence the function $(0, t) \ni s \mapsto v_{s}(\lambda)$ is strictly decreasing, thus we conclude (2.7) for $b \in \mathbb{R}_{+}$. If $b \in \mathbb{R}_{--}$, then $\theta_{0} \in \mathbb{R}_{++}$. Consequently, in case of $b \in \mathbb{R}_{--}$ and $\lambda \in\left(0, \theta_{0}\right)$ we have $v_{s}(\lambda)<v_{s}\left(\theta_{0}\right)=\theta_{0}$ for all $s \in \mathbb{R}_{+}$. In this case, using the differential equation (2.3) and the inequality $R(z)<0$ for all $z \in\left(0, \theta_{0}\right)$, we obtain $\frac{\partial}{\partial s} v_{s}(\lambda)=-R\left(v_{s}(\lambda)\right)>0$ for all $s \in \mathbb{R}_{+}$, hence the function $(0, t) \ni s \mapsto v_{s}(\lambda)$ is strictly increasing, thus we conclude (2.7) for $b \in \mathbb{R}_{--}$and $\lambda \in\left(0, \theta_{0}\right)$. In a similar way, in case of $b \in \mathbb{R}_{--}$and $\lambda \in\left(\theta_{0}, \infty\right)$ we have $v_{s}(\lambda)>v_{s}\left(\theta_{0}\right)=\theta_{0}$ for all $s \in \mathbb{R}_{+}$. In this case, using the differential equation (2.3) and the inequality $R(z)>0$ for all $z \in\left(\theta_{0}, \infty\right)$, we obtain $\frac{\partial}{\partial s} v_{s}(\lambda)=-R\left(v_{s}(\lambda)\right)<0$ for all $s \in \mathbb{R}_{+}$, hence the function $(0, t) \ni s \mapsto v_{s}(\lambda)$ is strictly decreasing, thus we conclude (2.7) for $b \in \mathbb{R}_{--}$and $\lambda \in\left(\theta_{0}, \infty\right)$ as well.

The form of the infinitesimal generator (2.5) can be checked similarly as in the proof of Theorem 2.1 of Barczy et al. 4], implying (iv).

For (v), let us fix $t \in \mathbb{R}_{++}$and put

$$
A_{t}:=\left\{\omega \in \Omega:[0, t] \ni s \mapsto Y_{s}(\omega) \text { is càdlàg and } Y_{s}(\omega) \in \mathbb{R}_{+} \text {for all } s \in[0, t]\right\} .
$$

Then, by (i), $\mathbb{P}\left(A_{t}\right)=1$ and for all $\omega \in A_{t}, \int_{0}^{t} Y_{s}(\omega) \mathrm{d} s=0$ if and only if $Y_{s}(\omega)=0$ for all $s \in[0, t)$. By (1.1),

$$
Y_{s}=Y_{0}+a s-b \int_{0}^{s} Y_{u} \mathrm{~d} u+\sigma \int_{0}^{s} \sqrt{Y_{u}} \mathrm{~d} W_{u}+\delta \int_{0}^{s} \sqrt[\alpha]{Y_{u-}} \mathrm{d} L_{u}, \quad s \in \mathbb{R}_{+},
$$

holds $\mathbb{P}$-almost surely. The stochastic integrals on the right hand side can be approximated as

$$
\begin{aligned}
& \sup _{s \in[0, t]}\left|\sum_{i=1}^{\lfloor n s\rfloor} \sqrt{Y_{\frac{i-1}{n}}}\left(W_{\frac{i}{n}}-W_{\frac{i-1}{n}}\right)-\int_{0}^{s} \sqrt{Y_{u}} \mathrm{~d} W_{u}\right| \stackrel{\mathbb{P}}{\longrightarrow} 0 \quad \text { as } \quad n \rightarrow \infty, \\
& \sup _{s \in[0, t] \mid}\left|\sum_{i=1}^{\lfloor n s\rfloor} \sqrt[\alpha]{\frac{Y_{\frac{i-1}{n}}}{\longrightarrow}}\left(L_{\frac{i}{n}}-L_{\frac{i-1}{n}}\right)-\int_{0}^{s} \sqrt[\alpha]{Y_{u-}} \mathrm{d} L_{u}\right| \stackrel{\mathbb{P}}{\longrightarrow} 0 \quad \text { as } \quad n \rightarrow \infty,
\end{aligned}
$$

see Jacod and Shiryaev [18, Theorem I.4.44]. Hence there exists a sequence $\left(n_{k}\right)_{k \in \mathbb{N}}$ of positive 
integers such that

$$
\begin{aligned}
& \sup _{s \in[0, t]}\left|\sum_{i=1}^{\left\lfloor n_{k} s\right\rfloor} \sqrt{Y_{\frac{i-1}{n_{k}}}}\left(W_{\frac{i}{n_{k}}}-W_{\frac{i-1}{n_{k}}}\right)-\int_{0}^{s} \sqrt{Y_{u}} \mathrm{~d} W_{u}\right| \rightarrow 0 \quad \text { as } \quad k \rightarrow \infty, \\
& \sup _{s \in[0, t]}\left|\sum_{i=1}^{\left\lfloor n_{k} s\right\rfloor} \sqrt[\alpha]{Y_{\frac{i-1}{n_{k}}}}\left(L_{\frac{i}{n_{k}}}-L_{\frac{i-1}{n_{k}}}\right)-\int_{0}^{s} \sqrt[\alpha]{Y_{u-}} \mathrm{d} L_{u}\right| \rightarrow 0 \quad \text { as } \quad k \rightarrow \infty
\end{aligned}
$$

hold $\mathbb{P}$-almost surely. Let us denote by $\tilde{A}_{t}$ the event on which the above two $\mathbb{P}$-almost sure convergences hold. Consequently, with the notation

$$
\tilde{\tilde{A}}_{t}:=\left\{\omega \in \Omega: \int_{0}^{t} Y_{s}(\omega) \mathrm{d} s=0\right\}
$$

we have

$$
\begin{aligned}
& \tilde{\tilde{A}}_{t} \cap \tilde{A}_{t} \cap A_{t} \subset \tilde{\tilde{A}}_{t} \cap\left\{\omega \in \Omega:\left(\int_{0}^{s} \sqrt{Y_{u}} \mathrm{~d} W_{u}\right)(\omega)=0, \quad\left(\int_{0}^{s} \sqrt[\alpha]{Y_{u-}} \mathrm{d} L_{u}\right)(\omega)=0 \text { for all } s \in[0, t)\right\} \\
& \subset \tilde{\tilde{A}}_{t} \cap\left\{\omega \in \Omega: Y_{s}(\omega)=Y_{0}(\omega)+a s \text { for all } s \in[0, t)\right\} \\
& \subset \tilde{\tilde{A}}_{t} \cap\left\{\omega \in \Omega: \int_{0}^{s}\left(Y_{0}(\omega)+a u\right) \mathrm{d} u=0 \text { for all } s \in[0, t)\right\} \\
& \subset \tilde{\tilde{A}}_{t} \cap\left\{\omega \in \Omega: Y_{0}(\omega) s+\frac{a s^{2}}{2}=0 \text { for all } s \in[0, t)\right\} \\
& \subset \tilde{\tilde{A}}_{t} \cap\left\{\omega \in \Omega: Y_{0}(\omega)=-\frac{a s}{2} \text { for all } s \in[0, t)\right\},
\end{aligned}
$$

where the last event has probability 0 , implying $\mathbb{P}\left(\int_{0}^{t} Y_{s}(\omega) \mathrm{d} s=0\right)=0$. Thus $\mathbb{P}\left(\int_{0}^{t} Y_{s}(\omega) \mathrm{d} s \in\right.$ $\left.\mathbb{R}_{++}\right)=0$, and hence we have $(\mathrm{v})$.

For (vi), see Proposition 3.7 in Jiao et al. [19].

Finally, we prove part (vii). First note that in case of $a=0, \quad\left(Y_{t}\right)_{t \in \mathbb{R}_{+}}$is a continuous time branching process (without immigration). If $b \in \mathbb{R}_{+}$, then by Corollary 3.9 in Li [25], $\mathbb{P}\left(\tau_{0}<\right.$ $\left.\infty \mid Y_{0}=y_{0}\right)=1$ for all $y_{0} \in \mathbb{R}_{++}$, since Condition 3.6 in Li [25] holds for all $\theta>0$ due to $\int_{\theta}^{\infty} \frac{1}{R(z)} \mathrm{d} z \leqslant \int_{\theta}^{\infty} \frac{2}{\sigma^{2} z^{2}} \mathrm{~d} z<\infty$. The last statement follows from the fact that in case of $a=0$ and $\mathbb{P}\left(Y_{0}=0\right)=1$, the pathwise unique non-negative strong solution of the SDE (1.1) is $Y_{t}=0$ for all $t \in \mathbb{R}_{+}$.

Note that, by Proposition 2.1, the process $\left(Y_{t}\right)_{t \in \mathbb{R}_{+}}$is a semimartingale, see, e.g., Jacod and Shiryaev [18, I.4.33]. Now we derive a so-called Grigelionis form for the semimartingale $\left(Y_{t}\right)_{t \in \mathbb{R}_{+}}$, see, e.g., Jacod and Shiryaev [18, III.2.23] or Jacod and Protter [17, Theorem 2.1.2].

2.2 Proposition. Let $\eta_{0}$ be a random variable independent of $\left(W_{t}\right)_{t \in \mathbb{R}_{+}}$and $\left(L_{t}\right)_{t \in \mathbb{R}_{+}}$satisfying $\mathbb{P}\left(\eta_{0} \in \mathbb{R}_{+}\right)=1$ and $\mathbb{E}\left(\eta_{0}\right)<\infty$. For $a \in \mathbb{R}_{+}, \quad b \in \mathbb{R}, \quad \sigma \in \mathbb{R}_{+}, \quad \delta \in \mathbb{R}_{++}, \quad$ and $\alpha \in(1,2)$, let $\left(Y_{t}\right)_{t \in \mathbb{R}_{+}}$be the unique strong solution of the $S D E$ (1.1) satisfying $\mathbb{P}\left(Y_{0}=\eta_{0}\right)=1$. Then the 
Grigelionis form of $\left(Y_{t}\right)_{t \in \mathbb{R}_{+}}$takes the form

$$
\begin{aligned}
Y_{t}= & Y_{0}+\int_{0}^{t}\left(a-b Y_{u}+\gamma \delta \sqrt[\alpha]{Y_{u}}\right) \mathrm{d} u+\int_{0}^{t}\left(\int_{\mathbb{R}}\left(h\left(z \delta \sqrt[\alpha]{Y_{u}}\right)-\delta \sqrt[\alpha]{Y_{u}} h(z)\right) m(\mathrm{~d} z)\right) \mathrm{d} u \\
& +\sigma \int_{0}^{t} \sqrt{Y_{u}} \mathrm{~d} W_{u} \\
& +\int_{0}^{t} \int_{\mathbb{R}} h\left(z \delta \sqrt[\alpha]{Y_{u-}}\right) \widetilde{\mu}^{L}(\mathrm{~d} u, \mathrm{~d} z)+\int_{0}^{t} \int_{\mathbb{R}}\left(z \delta \sqrt[\alpha]{Y_{u-}}-h\left(z \delta \sqrt[\alpha]{Y_{u-}}\right)\right) \mu^{L}(\mathrm{~d} u, \mathrm{~d} z)
\end{aligned}
$$

for $t \in \mathbb{R}_{+}$, where $h: \mathbb{R} \rightarrow[-1,1], h(z):=z \mathbb{1}_{[-1,1]}(z), \quad z \in \mathbb{R}$.

Proof. Using (2.1) and Proposition II.1.30 in Jacod and Shiryaev [18], we obtain

$$
\begin{aligned}
Y_{t}= & Y_{0}+\int_{0}^{t}\left(a-b Y_{u}\right) \mathrm{d} u+\int_{0}^{t} \sigma \sqrt{Y_{u}} \mathrm{~d} W_{u}+\delta \int_{0}^{t} \sqrt[\alpha]{Y_{u-}} \mathrm{d} L_{u} \\
= & Y_{0}+\int_{0}^{t}\left(a-b Y_{u}\right) \mathrm{d} u+\int_{0}^{t} \sigma \sqrt{Y_{u}} \mathrm{~d} W_{u}+\gamma \delta \int_{0}^{t} \sqrt[\alpha]{Y_{u-}} \mathrm{d} u \\
& +\delta \int_{0}^{t} \int_{\mathbb{R}} \sqrt[\alpha]{Y_{u-}} h(z) \widetilde{\mu}^{L}(\mathrm{~d} u, \mathrm{~d} z)+\delta \int_{0}^{t} \int_{\mathbb{R}} \sqrt[\alpha]{Y_{u-}}(z-h(z)) \mu^{L}(\mathrm{~d} u, \mathrm{~d} z)
\end{aligned}
$$

for $t \in \mathbb{R}_{+}$. In order to prove the statement, it is enough to show

$$
\begin{gathered}
\delta \int_{0}^{t} \int_{\mathbb{R}} \sqrt[\alpha]{Y_{u-}} h(z)\left(\mu^{L}(\mathrm{~d} u, \mathrm{~d} z)-\mathrm{d} u m(\mathrm{~d} z)\right)=I_{1}-I_{2} \\
\delta \int_{0}^{t} \int_{\mathbb{R}} \sqrt[\alpha]{Y_{u-}}(z-h(z)) \mu^{L}(\mathrm{~d} u, \mathrm{~d} z)=I_{3}+I_{4}
\end{gathered}
$$

with

$$
\begin{aligned}
& I_{1}:=\int_{0}^{t} \int_{\mathbb{R}} h\left(z \delta \sqrt[\alpha]{Y_{u-}}\right)\left(\mu^{L}(\mathrm{~d} u, \mathrm{~d} z)-\mathrm{d} u m(\mathrm{~d} z)\right), \\
& I_{2}:=\int_{0}^{t} \int_{\mathbb{R}}\left(h\left(z \delta \sqrt[\alpha]{Y_{u-}}\right)-\delta \sqrt[\alpha]{Y_{u-}} h(z)\right)\left(\mu^{L}(\mathrm{~d} u, \mathrm{~d} z)-\mathrm{d} u m(\mathrm{~d} z)\right), \\
& I_{3}:=\int_{0}^{t} \int_{\mathbb{R}}\left(z \delta \sqrt[\alpha]{Y_{u-}}-h\left(z \delta \sqrt[\alpha]{Y_{u-}}\right)\right) \mu^{L}(\mathrm{~d} u, \mathrm{~d} z), \\
& I_{4}:=\int_{0}^{t} \int_{\mathbb{R}}\left(h\left(z \delta \sqrt[\alpha]{Y_{u-}}\right)-\delta \sqrt[\alpha]{Y_{u-}} h(z)\right) \mu^{L}(\mathrm{~d} u, \mathrm{~d} z),
\end{aligned}
$$

and the equality

$$
I_{4}-I_{2}=I_{5} \quad \text { with } \quad I_{5}:=\int_{0}^{t}\left(\int_{\mathbb{R}}\left(h\left(z \delta \sqrt[\alpha]{Y_{u}}\right)-\delta \sqrt[\alpha]{Y_{u}} h(z)\right) m(\mathrm{~d} z)\right) \mathrm{d} u .
$$

For the equations (2.9), (2.10) and (2.11), it suffices to check the existence of $I_{2}, I_{3}$ and $I_{5}$.

First note that for every $s \in(0, \infty)$ we have

$$
h(s z)-\operatorname{sh}(z)= \begin{cases}s z \mathbb{1}_{\left\{1<|z| \leqslant \frac{1}{s}\right\}} & \text { if } s \in(0,1), \quad z \in \mathbb{R}, \\ 0 & \text { if } s=1, \quad z \in \mathbb{R}, \\ -s z \mathbb{1}_{\left\{\frac{1}{s}<|z| \leqslant 1\right\}} & \text { if } s \in(1, \infty), \quad z \in \mathbb{R} .\end{cases}
$$


The existence of $I_{2}$ will be a consequence of $I_{2}=I_{2,1}-I_{2,2}-I_{2,3}$ with

$$
\begin{aligned}
I_{2,1} & :=\int_{0}^{t} \int_{\mathbb{R}} \delta \sqrt[\alpha]{Y_{u-}} z \mathbb{1}_{\left\{1<|z| \leqslant \frac{1}{\delta \sqrt[\alpha]{Y_{u-}}}\right\}} \mathbb{1}_{\left\{\delta \sqrt[\alpha]{Y_{u-}} \in(0,1)\right\}} \mu^{L}(\mathrm{~d} u, \mathrm{~d} z), \\
I_{2,2} & :=\int_{0}^{t} \int_{\mathbb{R}} \delta \sqrt[\alpha]{Y_{u-}} z \mathbb{1}_{\left\{1<|z| \leqslant \frac{1}{\delta \sqrt[\alpha]{Y_{u-}}}\right\}} \mathbb{1}_{\left\{\delta \sqrt[\alpha]{Y_{u-}} \in(0,1)\right\}} \mathrm{d} u m(\mathrm{~d} z), \\
I_{2,3} & :=\int_{0}^{t} \int_{\mathbb{R}} \delta \sqrt[\alpha]{Y_{u-}} z \mathbb{1}_{\left\{\frac{1}{\delta \sqrt[\alpha]{Y_{u-}}}<|z| \leqslant 1\right\}} \mathbb{1}_{\left\{\delta \sqrt[\alpha]{Y_{u-}} \in(1, \infty)\right\}}\left(\mu^{L}(\mathrm{~d} u, \mathrm{~d} z)-\mathrm{d} u m(\mathrm{~d} z)\right),
\end{aligned}
$$

since on the set $\left\{Y_{u-}=0\right\}$, the integrand $h\left(z \delta \sqrt[\alpha]{Y_{u-}}\right)-\delta \sqrt[\alpha]{Y_{u-}} h(z)$ in $I_{2}$ takes value 0 . Here we have

$$
\left|I_{2,1}\right| \leqslant \int_{0}^{t} \int_{\mathbb{R}}\left|\delta \sqrt[\alpha]{Y_{u-}} z\right| \mathbb{1}_{\left\{1<|z| \leqslant \frac{1}{\delta \sqrt[\alpha]{Y_{u-}}}\right\}} \mathbb{1}_{\left\{\delta \sqrt[\alpha]{Y_{u-}} \in(0,1)\right\}} \mu^{L}(\mathrm{~d} u, \mathrm{~d} z) \leqslant \int_{0}^{t} \int_{\mathbb{R}} \mathbb{1}_{\{1<|z|\}} \mu^{L}(\mathrm{~d} u, \mathrm{~d} z)<\infty
$$

$\mathbb{P}$-almost surely, see, e.g., Sato [35, Lemma 20.1]. Moreover,

$$
\begin{aligned}
\left|I_{2,2}\right| & \leqslant \int_{0}^{t} \int_{\mathbb{R}}\left|\delta \sqrt[\alpha]{Y_{u-}} z\right| \mathbb{1}_{\left\{1<|z| \leqslant \frac{1}{\delta \sqrt[\alpha]{Y_{u-}}}\right.} \mathbb{1}_{\left\{\delta \sqrt[\alpha]{Y_{u-}} \in(0,1)\right\}} \mathrm{d} u m(\mathrm{~d} z) \\
& \leqslant \int_{0}^{t} \int_{\mathbb{R}} \mathbb{1}_{\{1<|z|\}} \mathrm{d} u m(\mathrm{~d} z)=\operatorname{tm}(\{z \in \mathbb{R}:|z|>1\})<\infty
\end{aligned}
$$

Further, the function $\Omega \times \mathbb{R}_{+} \times \mathbb{R} \ni(\omega, t, z) \mapsto h(z)$ belongs to $G_{\text {loc }}\left(\mu^{L}\right)$, see Jacod and Shiryaev [18, Definitions II.1.27, Theorem II.2.34]. We have $\left.\left|z \mathbb{1}_{\left\{\frac{1}{\delta \sqrt[\alpha]{Y_{u-}}}\right.}<\right| z \mid \leqslant 1\right\} \mathbb{1}_{\left\{\delta \sqrt[\alpha]{Y_{u-}} \in(1, \infty)\right\}}|\leqslant| h(z) \mid$, hence, by the definition of $G_{\text {loc }}\left(\mu^{L}\right)$, the function $\left.\left.\Omega \times \mathbb{R}_{+} \times \mathbb{R} \ni(\omega, t, z) \mapsto z \mathbb{1}_{\left\{\frac{1}{\delta \sqrt[\alpha]{Y_{u-}}}\right.}<|z| \leqslant 1\right\} \sqrt\left[\mathbb{1}_{\{\delta} \sqrt[\alpha]{Y_{u-}} \in(1, \infty)\right\}\right]{ }$ also belongs to $G_{\text {loc }}\left(\mu^{L}\right)$. By Jacod and Shiryaev [18, Proposition II.1.30], we conclude that the function $\Omega \times \mathbb{R}_{+} \times \mathbb{R} \ni(\omega, t, z) \mapsto \delta \sqrt[\alpha]{Y_{u-}} z \mathbb{1}_{\left\{\frac{1}{\delta \sqrt[\alpha]{Y_{u-}}}<|z| \leqslant 1\right\}} \mathbb{1}_{\left\{\delta \sqrt[\alpha]{Y_{u-}} \in(1, \infty)\right\}}$ also belongs to $G_{\text {loc }}\left(\mu^{L}\right)$, thus the integral $I_{2,3}$ exists, and hence we obtain the existence of $I_{2}$, and hence that of $I_{1}$.

Next observe that for the process $\zeta_{t}:=\delta \int_{0}^{t} \sqrt[\alpha]{Y_{u-}} \mathrm{d} L_{u}, \quad t \in \mathbb{R}_{+}$, we have $\Delta \zeta_{t}=\delta \sqrt[\alpha]{Y_{t-}} \Delta L_{t}$, $t \in \mathbb{R}_{+}$, following from (2.1) and Jacod and Shiryaev [18, Definitions II.1.27]. Consequently,

$$
\begin{aligned}
I_{3} & =\int_{0}^{t} \int_{\mathbb{R}} z \delta \sqrt[\alpha]{Y_{u-}} \mathbb{1}_{\left\{\left|z \delta \sqrt[\alpha]{Y_{u-}}\right|>1\right\}} \mu^{L}(\mathrm{~d} u, \mathrm{~d} z)=\sum_{u \in[0, t]} \Delta L_{u} \delta \sqrt[\alpha]{Y_{u-}} \mathbb{1}_{\left\{\left|\Delta L_{u} \delta \sqrt[\alpha]{Y_{u-}}\right|>1\right\}} \\
& =\sum_{u \in[0, t]} \Delta \zeta_{u} \mathbb{1}_{\left\{\left|\Delta \zeta_{u}\right|>1\right\}}
\end{aligned}
$$

is a finite sum, since the process $\left(\zeta_{t}\right)_{t \in[0, \infty)}$ admits càdlàg trajectories, hence there can be at most finitely many points $u \in[0, t]$ at which the absolute value $\left|\Delta \zeta_{u}\right|$ of the jump size $\Delta \zeta_{u}$ exceeds 1 , see, e.g., Billingsley [7, page 122]. Thus we obtain the existence of $I_{3}$, and hence that of $I_{4}$. 
Finally, we have

$$
\begin{aligned}
\left|I_{5}\right| \leqslant & \int_{0}^{t}\left(\int_{\mathbb{R}}\left|\delta \sqrt[\alpha]{Y_{u}} z\right| \mathbb{1}_{\left\{1<|z| \leqslant \frac{1}{\delta \sqrt[\alpha]{Y_{u}}}\right.} \mathbb{1}_{\left\{\delta \sqrt[\alpha]{Y_{u}} \in(0,1)\right\}} m(\mathrm{~d} z)\right) \mathrm{d} u \\
& +\int_{0}^{t}\left(\int_{\mathbb{R}}\left|\delta \sqrt[\alpha]{Y_{u}} z\right| \mathbb{1}_{\left\{\frac{1}{\delta \sqrt[\alpha]{Y_{u}}}<|z| \leqslant 1\right\}} \mathbb{1}_{\left\{\delta \sqrt[\alpha]{Y_{u}} \in(1, \infty)\right\}} m(\mathrm{~d} z)\right) \mathrm{d} u \\
\leqslant & \int_{0}^{t}\left(\int_{\mathbb{R}} \mathbb{1}_{\{1<|z|\}} m(\mathrm{~d} z)\right) \mathrm{d} u+\int_{0}^{t}\left(\int_{\mathbb{R}}\left|\delta \sqrt[\alpha]{Y_{u}} z\right|^{2} \mathbb{1}_{\{|z| \leqslant 1\}} m(\mathrm{~d} z)\right) \mathrm{d} u \\
= & t m(\{z \in \mathbb{R}:|z|>1\})+\int_{0}^{t} \delta^{2} Y_{u}^{\frac{2}{\alpha}} \mathrm{d} u \int_{-1}^{1}|z|^{2} m(\mathrm{~d} z)<\infty
\end{aligned}
$$

since $\int_{-1}^{1}|z|^{2} m(\mathrm{~d} z)=\int_{0}^{1} z^{2} C_{\alpha} z^{-1-\alpha} \mathrm{d} z=\frac{C_{\alpha}}{2-\alpha} \in \mathbb{R}_{++}$, hence we conclude the existence of $I_{5}$.

Next we present a result about the first moment of $\left(Y_{t}\right)_{t \in \mathbb{R}_{+}}$.

2.3 Proposition. Let $a \in \mathbb{R}_{+}, \quad b \in \mathbb{R}, \quad \sigma \in \mathbb{R}_{+}, \quad \delta \in \mathbb{R}_{++}$, and $\alpha \in(1,2)$. Let $\left(Y_{t}\right)_{t \in \mathbb{R}_{+}}$be the unique strong solution of the $S D E$ (1.1) satisfying $\mathbb{P}\left(Y_{0} \in \mathbb{R}_{+}\right)=1$ and $\mathbb{E}\left(Y_{0}\right)<\infty$. Then

$$
\mathbb{E}\left(Y_{t}\right)=\left\{\begin{array}{ll}
\mathrm{e}^{-b t}\left(\mathbb{E}\left(Y_{0}\right)-\frac{a}{b}\right)+\frac{a}{b} & \text { if } b \neq 0, \\
\mathbb{E}\left(Y_{0}\right)+a t & \text { if } b=0,
\end{array} \quad t \in \mathbb{R}_{+} .\right.
$$

Consequently, if $b \in \mathbb{R}_{++}$, then

$$
\lim _{t \rightarrow \infty} \mathbb{E}\left(Y_{t}\right)=\frac{a}{b}
$$

if $b=0$, then

if $b \in \mathbb{R}_{--}$, then

$$
\lim _{t \rightarrow \infty} t^{-1} \mathbb{E}\left(Y_{t}\right)=a
$$

$$
\lim _{t \rightarrow \infty} \mathrm{e}^{b t} \mathbb{E}\left(Y_{t}\right)=\mathbb{E}\left(Y_{0}\right)-\frac{a}{b}
$$

Proof. By Proposition 2.1, $\left(Y_{t}\right)_{t \in \mathbb{R}_{+}}$is CBI process with an infinitesimal generator given in (2.5). By the notations of Barczy et al. [5], this CBI process has parameters $(d, c, \beta, B, \nu, \mu)$, where $d=1$, $c=\frac{1}{2} \sigma^{2}, \quad \beta=a, \quad B=-b-\int_{0}^{\infty}(z-1)^{+} \mu(\mathrm{d} z), \quad \nu=0$ and $\mu=\delta^{\alpha} m$. Since $\mathbb{E}\left(Y_{0}\right)<\infty$ and the moment condition $\int_{\mathbb{R} \backslash\{0\}}|z| \mathbb{1}_{\{|z| \geqslant 1\}} \nu(\mathrm{d} z)<\infty$ trivially holds, we may apply formula (3.1.11) in Li [27] or Lemma 3.4 and (2.14) in Barczy et al. [5] with $\widetilde{B}=B+\int_{0}^{\infty}(z-1)^{+} \mu(\mathrm{d} z)=-b$ and $\widetilde{\beta}=\beta+\int_{\mathbb{R} \backslash\{0\}} z \nu(\mathrm{d} z)=a$ yielding that

$$
\mathbb{E}\left(Y_{t}\right)=\mathrm{e}^{t \widetilde{B}} \mathbb{E}\left(Y_{0}\right)+\left(\int_{0}^{t} \mathrm{e}^{u \widetilde{B}} \mathrm{~d} u\right) \widetilde{\beta}
$$

This implies (2.13) and the other parts of the assertion.

Based on the asymptotic behavior of the expectations $\mathbb{E}\left(Y_{t}\right)$ as $t \rightarrow \infty$, we introduce a classification of the stable CIR model given by the SDE (1.1).

2.4 Definition. Let $a \in \mathbb{R}_{+}, \quad b \in \mathbb{R}, \quad \sigma \in \mathbb{R}_{+}, \quad \delta \in \mathbb{R}_{++}$, and $\alpha \in(1,2)$. Let $\left(Y_{t}\right)_{t \in \mathbb{R}_{+}}$be the unique strong solution of the $S D E$ (1.1) satisfying $\mathbb{P}\left(Y_{0} \in \mathbb{R}_{+}\right)=1$ and $\mathbb{E}\left(Y_{0}\right)<\infty$. We call $\left(Y_{t}\right)_{t \in \mathbb{R}_{+}}$subcritical, critical or supercritical if $b \in \mathbb{R}_{++}, \quad b=0$ or $b \in \mathbb{R}_{--}$, respectively. 
The following result states the existence of a unique stationary distribution for the process $\left(Y_{t}\right)_{t \in \mathbb{R}_{+}}$ in the subcritical and critical cases, and the exponential ergodicity in the subcritical case.

2.5 Theorem. Let $a \in \mathbb{R}_{+}, \quad b \in \mathbb{R}_{+}, \quad \sigma \in \mathbb{R}_{+}, \quad \delta \in \mathbb{R}_{++}$, and $\alpha \in(1,2)$. Let $\left(Y_{t}\right)_{t \in \mathbb{R}_{+}}$be the unique strong solution of the $S D E(1.1)$ satisfying $\mathbb{P}\left(Y_{0} \in \mathbb{R}_{+}\right)=1$ and $\mathbb{E}\left(Y_{0}\right)<\infty$.

(i) Then $\left(Y_{t}\right)_{t \in \mathbb{R}_{+}}$converges in law to its unique stationary distribution $\pi$ having Laplace transform

$$
\int_{0}^{\infty} \mathrm{e}^{-\lambda y} \pi(\mathrm{d} y)=\exp \left\{-\int_{0}^{\lambda} \frac{F(x)}{R(x)} \mathrm{d} x\right\}=\exp \left\{-\int_{0}^{\lambda} \frac{a x}{\frac{\sigma^{2}}{2} x^{2}+\frac{\delta^{\alpha}}{\alpha} x^{\alpha}+b x} \mathrm{~d} x\right\}
$$

for $\lambda \in \mathbb{R}_{+}$. Especially, in case of $b=0$ and $\sigma=0, \quad \pi$ is a strictly $(2-\alpha)$-stable distribution with no negative jumps. Moreover, the expectation of $\pi$ is given by

$$
\int_{0}^{\infty} y \pi(\mathrm{d} y)= \begin{cases}0 & \text { if } a=0 \text { and } b=0, \\ \frac{a}{b} \in \mathbb{R}_{+} & \text {if } b \in \mathbb{R}_{++}, \\ +\infty & \text { if } a \in \mathbb{R}_{++} \text {and } b=0 .\end{cases}
$$

(ii) If, in addition, $a \in \mathbb{R}_{++}$and $b \in \mathbb{R}_{++}$, then the process $\left(Y_{t}\right)_{t \in \mathbb{R}_{+}}$is exponentially ergodic, i.e., there exist constants $C \in \mathbb{R}_{++}$and $D \in \mathbb{R}_{++}$such that

$$
\left\|\mathbb{P}_{Y_{t} \mid Y_{0}=y}-\pi\right\|_{\mathrm{TV}} \leqslant C(y+1) \mathrm{e}^{-D t}, \quad t \in \mathbb{R}_{+}, \quad y \in \mathbb{R}_{+},
$$

where $\|\mu\|_{\mathrm{TV}}$ denotes the total-variation norm of a signed measure $\mu$ on $\mathbb{R}_{+}$defined by $\|\mu\|_{\mathrm{TV}}:=\sup _{A \in \mathcal{B}\left(\mathbb{R}_{+}\right)}|\mu(A)|$, and $\mathbb{P}_{Y_{t} \mid Y_{0}=y}$ is the conditional distribution of $Y_{t}$ with respect to the condition $Y_{0}=y$. As a consequence, for all Borel measurable functions $f: \mathbb{R}_{+} \rightarrow \mathbb{R}$ with $\int_{0}^{\infty}|f(y)| \pi(\mathrm{d} y)<\infty$, we have

$$
\frac{1}{T} \int_{0}^{T} f\left(Y_{s}\right) \mathrm{d} s \stackrel{\text { a.s. }}{\longrightarrow} \int_{0}^{\infty} f(y) \pi(\mathrm{d} y) \quad \text { as } T \rightarrow \infty .
$$

Proof. The weak convergence of $Y_{t}$ towards $\pi$ as $t \rightarrow \infty$, and the fact that $\pi$ is a stationary distribution for $\left(Y_{t}\right)_{t \in \mathbb{R}_{+}}$follow immediately from Li [25, Theorem 3.20 and the paragraph after Corollary 3.21], since $R(z)=\frac{\sigma^{2}}{2} z^{2}+\frac{\delta^{\alpha}}{\alpha} z^{\alpha}+b z \in \mathbb{R}_{++}, \quad z \in \mathbb{R}_{++}$, and condition (3.30) in Li [25] is satisfied. Indeed, for all $\lambda \in \mathbb{R}_{++}$,

$$
\int_{0}^{\lambda} \frac{F(z)}{R(z)} \mathrm{d} z=\int_{0}^{\lambda} \frac{a z}{\frac{\sigma^{2}}{2} z^{2}+\frac{\delta^{\alpha}}{\alpha} z^{\alpha}+b z} \mathrm{~d} z \leqslant \frac{a \alpha}{\delta^{\alpha}} \int_{0}^{\lambda} z^{1-\alpha} \mathrm{d} z=\frac{a \alpha \lambda^{2-\alpha}}{\delta^{\alpha}(2-\alpha)}<\infty .
$$

We note that Li and Ma [26, Proposition 2.2] contains the above considerations in case of $b \in \mathbb{R}_{++}$. The uniqueness of a stationary distribution in (i) follows from, e.g., page 80 in Keller-Ressel 23. Namely, let us assume that there exists another stationary distribution $\pi^{\prime}$ for $\left(Y_{t}\right)_{t \in \mathbb{R}_{+}}$, and let $\left(Y_{t}^{\prime}\right)_{t \in \mathbb{R}_{+}}$be the unique strong solution of the $\operatorname{SDE}(1.1)$ with $a \in \mathbb{R}_{+}, \quad b \in \mathbb{R}_{+}, \quad \sigma \in \mathbb{R}_{+}$, and $\delta \in \mathbb{R}_{++}$satisfying $\mathcal{L}\left(Y_{0}^{\prime}\right)=\pi^{\prime}$, where $\mathcal{L}\left(Y_{0}^{\prime}\right)$ denotes the law of $Y_{0}^{\prime}$. Then, by part (iii) of Proposition 2.1, for all $\lambda \in \mathbb{R}_{+}$,

$$
\begin{aligned}
\lim _{t \rightarrow \infty} \mathbb{E}\left(\mathrm{e}^{-\lambda Y_{t}^{\prime}}\right) & =\lim _{t \rightarrow \infty} \mathbb{E}\left(\mathbb{E}\left(\mathrm{e}^{-\lambda Y_{t}^{\prime}} \mid Y_{0}^{\prime}\right)\right)=\lim _{t \rightarrow \infty} \mathbb{E}\left(\exp \left\{-Y_{0}^{\prime} v_{t}(\lambda)+\int_{\lambda}^{v_{t}(\lambda)} \frac{F(z)}{R(z)} \mathrm{d} z\right\}\right) \\
& =\mathbb{E}\left(\exp \left\{-\int_{0}^{\lambda} \frac{F(z)}{R(z)} \mathrm{d} z\right\}\right)=\exp \left\{-\int_{0}^{\lambda} \frac{F(z)}{R(z)} \mathrm{d} z\right\},
\end{aligned}
$$


where the last but one step follows by the dominated convergence theorem and the fact that $\lim _{t \rightarrow \infty} v_{t}(\lambda)=0, \quad \lambda \in \mathbb{R}_{+}$(see, e.g., the proof of Theorem 3.20 in Li [25]). Since $\mathcal{L}\left(Y_{t}^{\prime}\right)=\pi^{\prime}$, $t \in \mathbb{R}_{+}$, we have $\int_{0}^{\infty} \mathrm{e}^{-\lambda z} \pi^{\prime}(\mathrm{d} z)=\exp \left\{-\int_{0}^{\lambda} \frac{F(z)}{R(z)} \mathrm{d} z\right\}, \quad \lambda \in \mathbb{R}_{+}$, yielding that $\int_{0}^{\infty} \mathrm{e}^{-\lambda z} \pi^{\prime}(\mathrm{d} z)=$ $\int_{0}^{\infty} \mathrm{e}^{-\lambda z} \pi(\mathrm{d} z), \quad \lambda \in \mathbb{R}_{+}$. By the uniqueness of Laplace transform, we get $\pi=\pi^{\prime}$, as desired.

Further, in case of $b=0$ and $\sigma=0$, by (2.15), we have

$$
\int_{0}^{\infty} \mathrm{e}^{-\lambda y} \pi(\mathrm{d} y)=\exp \left\{-\int_{0}^{\lambda} \frac{a x}{\frac{\delta^{\alpha}}{\alpha} x^{\alpha}} \mathrm{d} x\right\}=\exp \left\{-\frac{a \alpha}{(2-\alpha) \delta^{\alpha}} \lambda^{2-\alpha}\right\}, \quad \lambda \in \mathbb{R}_{+},
$$

so $\pi$ is a strictly $(2-\alpha)$-stable distribution with no negative jumps. Finally, again by (2.15),

$$
\int_{0}^{\infty} y \pi(\mathrm{d} y)=\lim _{\lambda \downarrow 0} \frac{a \lambda}{\frac{\sigma^{2}}{2} \lambda^{2}+\frac{\delta^{\alpha}}{\alpha} \lambda^{\alpha}+b \lambda}= \begin{cases}0 & \text { if } a=0 \text { and } b=0 \\ \frac{a}{b} \in \mathbb{R}_{+} & \text {if } b \in \mathbb{R}_{++}, \\ +\infty & \text { if } a \in \mathbb{R}_{++} \text {and } b=0 .\end{cases}
$$

For part (ii), we can use Theorem 2.5 in $\mathrm{Li}$ and Ma [26]. We only have to check Condition 2.1 of $\mathrm{Li}$ and Ma [26], namely, we have to show the existence of some constant $\theta \in \mathbb{R}_{++}$such that $R(z) \in \mathbb{R}_{++}$ for $z>\theta$ and $\int_{\theta}^{\infty} \frac{1}{R(z)} \mathrm{d} z<\infty$. Here $R(z) \in \mathbb{R}_{++}$for all $z \in \mathbb{R}_{++}$(due to $b \in \mathbb{R}_{++}$), and, e.g., with $\theta=1$,

$$
\int_{1}^{\infty} \frac{1}{R(z)} \mathrm{d} z \leqslant \frac{\alpha}{\delta^{\alpha}} \int_{1}^{\infty} \frac{1}{z^{\alpha}} \mathrm{d} z=\frac{\alpha}{(\alpha-1) \delta^{\alpha}}<\infty
$$

In case of $\sigma=0$, the exponential ergodicity of $\left(Y_{t}\right)_{t \in \mathbb{R}_{+}}$also follows by Theorem 6.1 in Jin et al. [21]. Convergence (2.17) follows, e.g., from the discussion after Proposition 2.5 in Bhattacharya [8].

2.6 Remark. In what follows, in case of $\sigma \in \mathbb{R}_{++}$, we present another (and more detailed) proof for the convergence $Y_{t} \stackrel{\mathcal{D}}{\longrightarrow} \pi$ as $t \rightarrow \infty$ in Theorem 2.5 giving more insight as well. It is enough to consider the case of $\mathbb{P}\left(Y_{0}=y_{0}\right)=1$ with some $y_{0} \in \mathbb{R}_{+}$. Using (2.2), we have

$$
\mathbb{E}\left(\mathrm{e}^{-\lambda Y_{t}}\right)=\exp \left\{-y_{0} v_{t}(\lambda)-a \int_{0}^{t} v_{s}(\lambda) \mathrm{d} s\right\}
$$

for $t \in \mathbb{R}_{+}$and $\lambda \in \mathbb{R}_{+}$, where the function $\mathbb{R}_{+} \ni \lambda \mapsto v_{t}(\lambda) \in \mathbb{R}_{+}$is given by (2.3). First, we show that $\lim _{t \rightarrow \infty} v_{t}(\lambda)=0$. The proof is based on the following version of the comparison theorem (see, e.g., Lemma C.3. in Filipović et al. [14] or Amann [1, Lemma 16.4]): if $S: \mathbb{R}_{+} \times \mathbb{R} \rightarrow \mathbb{R}$ is a continuous function which is locally Lipschitz continuous in its second variable and $p, q: \mathbb{R}_{+} \rightarrow \mathbb{R}$ are differentiable functions satisfying

$$
\begin{array}{ll}
p^{\prime}(s) \leqslant S(s, p(s)), & s \in \mathbb{R}_{+}, \\
q^{\prime}(s)=S(s, q(s)), & s \in \mathbb{R}_{+}, \\
p(0) \leqslant q(0), &
\end{array}
$$

then $p(s) \leqslant q(s)$ for all $s \in \mathbb{R}_{+}$. By choosing $S: \mathbb{R}_{+} \times \mathbb{R} \rightarrow \mathbb{R}, S(s, x):=-\frac{\sigma^{2}}{2} x^{2}, \quad(s, x) \in \mathbb{R}_{+} \times \mathbb{R}$, the comparison theorem yields that

$$
0 \leqslant v_{s}(\lambda) \leqslant f(s), \quad s \in \mathbb{R}_{+},
$$


where $f: \mathbb{R}_{+} \rightarrow \mathbb{R}_{+}$is the unique locally bounded solution to the differential equation

$$
f^{\prime}(s)=S(s, f(s))=-\frac{\sigma^{2}}{2} f(s)^{2}, \quad s \in \mathbb{R}_{+} \quad \text { with } \quad f(0)=\lambda .
$$

The solution of this separable differential equation takes the form

$$
f(s)=\frac{\lambda}{1+\frac{\sigma^{2} \lambda}{2} s}, \quad s \in \mathbb{R}_{+} .
$$

Hence, using $\sigma \in \mathbb{R}_{++}$, we readily have $\lim _{t \rightarrow \infty} f(t)=0$, which, by (2.18), yields that $\lim _{t \rightarrow \infty} v_{t}(\lambda)=0$ for all $\lambda \in \mathbb{R}_{+}$, as desired. Further, by (2.4), we have

$$
\lim _{t \rightarrow \infty} \int_{0}^{t} v_{s}(\lambda) \mathrm{d} s=-\lim _{t \rightarrow \infty} \int_{\lambda}^{v_{t}(\lambda)} \frac{z}{\frac{\sigma^{2}}{2} z^{2}+\frac{\delta^{\alpha}}{\alpha} z^{\alpha}+b z} \mathrm{~d} z, \quad \lambda \in \mathbb{R}_{+} .
$$

Then, by the continuity of the integral upper limit function, we have

$$
\lim _{t \rightarrow \infty} \int_{0}^{t} v_{s}(\lambda) \mathrm{d} s=\int_{0}^{\lambda} \frac{z}{\frac{\sigma^{2}}{2} z^{2}+\frac{\delta^{\alpha}}{\alpha} z^{\alpha}+b z} \mathrm{~d} z, \quad \lambda \in \mathbb{R}_{+},
$$

where the integral on the right hand side is well-defined, since

$$
\int_{0}^{\lambda} \frac{z}{\frac{\sigma^{2}}{2} z^{2}+\frac{\delta^{\alpha}}{\alpha} z^{\alpha}+b z} \mathrm{~d} z \leqslant \int_{0}^{\lambda} \frac{z}{\frac{\delta^{\alpha}}{\alpha} z^{\alpha}} \mathrm{d} z=\frac{\alpha}{\delta^{\alpha}} \int_{0}^{\lambda} \frac{1}{z^{\alpha-1}} \mathrm{~d} z=\frac{\alpha \lambda^{2-\alpha}}{\delta^{\alpha}(2-\alpha)}<\infty .
$$

Consequently, by continuity theorem, we have (2.15) in case of $\sigma \in \mathbb{R}_{++}$.

Next we give a statistic for $\sigma^{2}$ using continuous time observations $\left(Y_{t}\right)_{t \in[0, T]}$ with an arbitrary $T \in \mathbb{R}_{++}$. Due to this result we do not consider the estimation of the parameter $\sigma$, it is supposed to be known. The parameter $\sigma$ is a parameter for the diffusion part related to $W$, and, in general, the estimation of this kind of parameter is possible using an arbitrarily short (continuous time) observation of the underlying process (at least theoretically), and that's why authors suppose this to be known. In the forthcoming Remark 2.7, we demonstrate that this also holds for our model.

2.7 Remark. Let $a \in \mathbb{R}_{+}, \quad b \in \mathbb{R}, \quad \sigma \in \mathbb{R}_{+}, \quad \delta \in \mathbb{R}_{++}$, and $\alpha \in(1,2)$. Let $\left(Y_{t}\right)_{t \in \mathbb{R}_{+}}$be the unique strong solution of the SDE (1.1) satisfying $\mathbb{P}\left(Y_{0} \in \mathbb{R}_{+}\right)=1$ and $\mathbb{E}\left(Y_{0}\right)<\infty$. The Grigelionis representation given in (2.8) implies that the continuous martingale part $Y^{\text {cont }}$ of $Y$ is $Y_{t}^{\text {cont }}=\sigma \int_{0}^{t} \sqrt{Y_{u}} \mathrm{~d} W_{u}, t \in \mathbb{R}_{+}$, see Jacod and Shiryaev [18, III.2.28 Remarks, part 1)]. Consequently, the (predictable) quadratic variation process of $Y^{\text {cont }}$ is $\left\langle Y^{\text {cont }}\right\rangle_{t}=\sigma^{2} \int_{0}^{t} Y_{u} \mathrm{~d} u, t \in \mathbb{R}_{+}$. Suppose that we have $\mathbb{P}\left(Y_{0} \in \mathbb{R}_{++}\right)=1$ or $a \in \mathbb{R}_{++}$. Then for all $T \in \mathbb{R}_{++}$, we have

$$
\sigma^{2}=\frac{\left\langle Y^{\mathrm{cont}}\right\rangle_{T}}{\int_{0}^{T} Y_{u} \mathrm{~d} u}=: \widehat{\sigma}_{T}^{2}
$$

since, due to (v) of Proposition 2.1. $\mathbb{P}\left(\int_{0}^{T} Y_{u} \mathrm{~d} u \in \mathbb{R}_{++}\right)=1$. We note that $\widehat{\sigma}_{T}^{2}$ is a statistic, i.e., there exists a measurable function $\Xi: D([0, T], \mathbb{R}) \rightarrow \mathbb{R}$ such that $\widehat{\sigma}_{T}^{2}=\Xi\left(\left(Y_{u}\right)_{u \in[0, T]}\right)$, where $D([0, T], \mathbb{R})$ denotes the space of real-valued càdlàg functions defined on $[0, T]$, since

$$
\frac{1}{\frac{1}{n} \sum_{i=1}^{\lfloor n T\rfloor} Y_{\frac{i-1}{n}}}\left(\sum_{i=1}^{\lfloor n T\rfloor}\left(Y_{\frac{i}{n}}-Y_{\frac{i-1}{n}}\right)^{2}-\sum_{u \in[0, T]}\left(\Delta Y_{u}\right)^{2}\right) \stackrel{\mathbb{P}}{\longrightarrow} \widehat{\sigma}_{T}^{2} \quad \text { as } n \rightarrow \infty,
$$


where the convergence in (2.20) holds $\mathbb{P}$-almost surely along a suitable subsequence, the members of the sequence in (2.20) are measurable functions of $\left(Y_{u}\right)_{u \in[0, T]}$, and one can use Theorems 4.2 .2 and 4.2.8 in Dudley [11. Next we prove (2.20). By Theorem I.4.47 a) in Jacod and Shiryaev [18,

$$
\sum_{i=1}^{\lfloor n T\rfloor}\left(Y_{\frac{i}{n}}-Y_{\frac{i-1}{n}}\right)^{2} \stackrel{\mathbb{P}}{\longrightarrow}[Y]_{T} \quad \text { as } n \rightarrow \infty, \quad T \in \mathbb{R}_{+},
$$

where $\left([Y]_{t}\right)_{t \in \mathbb{R}_{+}}$denotes the quadratic variation process of the semimartingale $Y$. By Theorem I.4.52 in Jacod and Shiryaev 18,

$$
[Y]_{T}=\left\langle Y^{\text {cont }}\right\rangle_{T}+\sum_{u \in[0, T]}\left(\Delta Y_{u}\right)^{2}, \quad T \in \mathbb{R}_{+} .
$$

Consequently, for all $T \in \mathbb{R}_{+}$, we have

$$
\sum_{i=1}^{\lfloor n T\rfloor}\left(Y_{\frac{i}{n}}-Y_{\frac{i-1}{n}}\right)^{2}-\sum_{u \in[0, T]}\left(\Delta Y_{u}\right)^{2} \stackrel{\mathbb{P}}{\longrightarrow}\left\langle Y^{\mathrm{cont}}\right\rangle_{T} \quad \text { as } n \rightarrow \infty .
$$

Moreover, for all $T \in \mathbb{R}_{+}$, we have

$$
\frac{1}{n} \sum_{i=1}^{\lfloor n T\rfloor} Y_{\frac{i-1}{n}} \stackrel{\mathbb{P}}{\longrightarrow} \int_{0}^{T} Y_{u} \mathrm{~d} u \quad \text { as } n \rightarrow \infty,
$$

see Proposition I.4.44 in Jacod and Shiryaev [18. Hence (2.20) follows by the fact that convergence in probability is closed under multiplication. Finally, we note that $T$ is fixed above, and it is enough to know any observation $\left(Y_{t}\right)_{t \in[0, T]}$ to carry out the above calculations, where $T>0$ can be arbitrarily small.

\section{Joint Laplace transform of $Y_{t}$ and $\int_{0}^{t} Y_{s} \mathrm{~d} s$}

Using Theorem 4.10 in Keller-Ressel [22] we derive a formula for the joint Laplace transform of $Y_{t}$ and $\int_{0}^{t} Y_{s} \mathrm{~d} s$, where $t \in \mathbb{R}_{+}$. We note that this form of the joint Laplace transform in question is a consequence of Theorem 5.3 in Filipović [13] and a special case of Proposition 3.3 in Jiao et al. [19] as well.

3.1 Theorem. Let $a \in \mathbb{R}_{+}, \quad b \in \mathbb{R}, \quad \sigma \in \mathbb{R}_{+}, \quad \delta \in \mathbb{R}_{++}$, and $\alpha \in(1,2)$. Let $\left(Y_{t}\right)_{t \in \mathbb{R}_{+}}$be the unique strong solution of the $S D E(1.1)$ satisfying $\mathbb{P}\left(Y_{0}=y_{0}\right)=1$ with some $y_{0} \in \mathbb{R}_{+}$. Then for all $u, v \in \mathbb{R}_{-}$,

$$
\mathbb{E}\left[\exp \left\{u Y_{t}+v \int_{0}^{t} Y_{s} \mathrm{~d} s\right\}\right]=\exp \left\{y_{0} \psi_{u, v}(t)+a \int_{0}^{t} \psi_{u, v}(s) \mathrm{d} s\right\}, \quad t \in \mathbb{R}_{+},
$$

where the function $\psi_{u, v}: \mathbb{R}_{+} \rightarrow \mathbb{R}_{-}$is the unique locally bounded solution to the differential equation

$$
\psi_{u, v}^{\prime}(t)=\frac{\sigma^{2}}{2} \psi_{u, v}(t)^{2}+\frac{\delta^{\alpha}}{\alpha}\left(-\psi_{u, v}(t)\right)^{\alpha}-b \psi_{u, v}(t)+v, \quad t \in \mathbb{R}_{+}, \quad \psi_{u, v}(0)=u .
$$

Further, if $(u, v) \neq(0,0)$, then $\psi_{u, v}(t) \in \mathbb{R}_{--}, \quad t \in \mathbb{R}_{++}$, and if $(u, v)=(0,0)$, then $\psi_{u, v}(t)=0$, $t \in \mathbb{R}_{+}$. 
Proof. By Theorem 4.10 in Keller-Ressel [22], $\left(Y_{t}, \int_{0}^{t} Y_{s} \mathrm{~d} s\right)_{t \in \mathbb{R}_{+}}$is a 2-dimensional CBI process with branching mechanism $\widetilde{R}\left(z_{1}, z_{2}\right)=\left(\widetilde{R}_{1}\left(z_{1}, z_{2}\right), \widetilde{R}_{2}\left(z_{1}, z_{2}\right)\right), z_{1}, z_{2} \in \mathbb{R}_{+}$, with

$$
\widetilde{R}_{1}\left(z_{1}, z_{2}\right)=R\left(z_{1}\right)-z_{2}, \quad \widetilde{R}_{2}\left(z_{1}, z_{2}\right)=0, \quad z_{1}, z_{2} \in \mathbb{R}_{+},
$$

and with immigration mechanism $\widetilde{F}\left(z_{1}, z_{2}\right)=F\left(z_{1}\right), z_{1}, z_{2} \in \mathbb{R}_{+}$, where $R$ and $F$ are given in Proposition 2.1. Consequently, by Theorem 2.7 of Duffie et al. [12] (see also Barczy et al. [5, Theorem 2.4]), we have

$$
\begin{aligned}
\mathbb{E}\left[\exp \left\{u Y_{t}+v \int_{0}^{t} Y_{s} \mathrm{~d} s\right\}\right] & =\exp \left\{y_{0} \psi_{u, v}(t)-\int_{0}^{t} \widetilde{F}\left(-\psi_{u, v}(s),-\varphi_{u, v}(s)\right) \mathrm{d} s\right\} \\
& =\exp \left\{y_{0} \psi_{u, v}(t)+a \int_{0}^{t} \psi_{u, v}(s) \mathrm{d} s\right\}, \quad t \in \mathbb{R}_{+}
\end{aligned}
$$

where the function $\left(\psi_{u, v}, \varphi_{u, v}\right): \mathbb{R}_{+} \rightarrow \mathbb{R}_{-}^{2}$ is the unique locally bounded solution to the system of differential equations

$$
\left\{\begin{array}{l}
\psi_{u, v}^{\prime}(t)=\widetilde{R}_{1}\left(-\psi_{u, v}(t),-\varphi_{u, v}(t)\right)=\frac{\sigma^{2}}{2} \psi_{u, v}(t)^{2}+\frac{\delta^{\alpha}}{\alpha}\left(-\psi_{u, v}(t)\right)^{\alpha}-b \psi_{u, v}(t)+\varphi_{u, v}(t), \quad t \in \mathbb{R}_{+}, \\
\varphi_{u, v}^{\prime}(t)=\widetilde{R}_{2}\left(-\psi_{u, v}(t),-\varphi_{u, v}(t)\right)=0, \quad t \in \mathbb{R}_{+},
\end{array}\right.
$$

with initial values $\psi_{u, v}(0)=u, \varphi_{u, v}(0)=v$. Clearly, $\varphi_{u, v}(t)=v, t \in \mathbb{R}_{+}$, hence we obtain

$$
\psi_{u, v}^{\prime}(t)=\frac{\sigma^{2}}{2} \psi_{u, v}(t)^{2}+\frac{\delta^{\alpha}}{\alpha}\left(-\psi_{u, v}(t)\right)^{\alpha}-b \psi_{u, v}(t)+v, \quad t \in \mathbb{R}_{+}, \quad \psi_{u, v}(0)=u,
$$

as desired.

If $(u, v)=(0,0)$, then, since the identically zero function is a (locally bounded) solution to (3.1), by the unicity of such a solution, we have $\psi_{0,0}(t)=0, t \in \mathbb{R}_{+}$. If $(u, v) \neq(0,0)$, then, on the contrary, let us suppose that there exists an $t_{0} \in \mathbb{R}_{++}$such that $\psi_{u, v}\left(t_{0}\right)=0$. Let $t_{*}:=\inf \left\{t \in \mathbb{R}_{++}: \psi_{u, v}(t)=0\right\}$. Then $t_{*}<\infty, \psi_{u, v}(t)<0$ for all $t \in\left[0, t_{*}\right)$, and $\psi_{u, v}\left(t_{*}\right)=0$. If $t_{*}=0$, then $0=\psi_{u, v}\left(t_{*}\right)=\psi_{u, v}(0)=u$, and hence $v \in \mathbb{R}_{--}$. Further, there exists a sequence $\left(t_{n}\right)_{n \in \mathbb{N}}$ such that $t_{n} \in \mathbb{R}_{++}, \quad n \in \mathbb{N}, t_{n} \downarrow 0=t_{*}$ as $n \rightarrow \infty$, and $\psi_{0, v}\left(t_{n}\right)=0, n \in \mathbb{N}$. Consequently, using that a locally bounded solution to (3.1) is unique, $\psi_{0, v}\left(k t_{n}\right)=\psi_{0, v}(0)=0$, $k, n \in \mathbb{N}$. Since $t_{n} \downarrow 0$ as $n \rightarrow \infty$, for all $t \in \mathbb{R}_{++}$, there exists a sequence $\left(k_{n}\right)_{n \in \mathbb{N}}$ such that $k_{n} \in \mathbb{N}, \quad n \in \mathbb{N}$, and $k_{n} t_{n} \rightarrow t$ as $n \rightarrow \infty$ (one can choose $k_{n}:=\left\lfloor\frac{t}{t_{n}}\right\rfloor, n \in \mathbb{N}$ ). Since $\psi_{0, v}$ is continuous, we have $\psi_{0, v}(t)=0, t \in \mathbb{R}_{+}$, yielding us to a contradiction (due to $v \in \mathbb{R}_{--}$). So, if $t_{*}=0$, then $\psi_{u, v}(t)<0, t>0$, as desired. In the sequel, let us assume that $t_{*}>0$. On the one hand, $\psi_{u, v}^{\prime}\left(t_{*}\right) \geqslant 0$, since

$$
\psi_{u, v}^{\prime}\left(t_{*}\right)=\lim _{h \uparrow 0} \frac{\psi_{u, v}\left(t_{*}+h\right)-\psi_{u, v}\left(t_{*}\right)}{h}=\lim _{h \uparrow 0} \frac{1}{h} \psi_{u, v}\left(t_{*}+h\right),
$$

where $h<0$ and $\psi_{u, v}\left(t_{*}+h\right)<0$ yield $\frac{1}{h} \psi_{u, v}\left(t_{*}+h\right)>0$. On the other hand, by (3.1), $\psi_{u, v}^{\prime}\left(t_{*}\right)=v \leqslant 0$, yielding that $v=0$. Consequently, if $t_{*}>0$, then (3.1) takes the form

$$
\psi_{u, v}^{\prime}(t)=\frac{\sigma^{2}}{2} \psi_{u, v}(t)^{2}+\frac{\delta^{\alpha}}{\alpha}\left(-\psi_{u, v}(t)\right)^{\alpha}-b \psi_{u, v}(t), \quad t \in \mathbb{R}_{+},
$$


where $\psi_{u, v}(0)=u$ and $\psi_{u, v}\left(t_{*}\right)=0$. By the uniqueness of a locally bounded solution of (3.1), $\psi_{u, v}(t)=0$ for all $t \geqslant t_{*}$, and hence $\psi_{u, v}(t)=0$ for all $t \geqslant 0$. Indeed, let $\widetilde{\psi}_{u, v}(\tau):=\psi_{u, v}(-\tau)$, $\tau \leqslant 0$, and $\tau^{*}:=-t_{*}$. Then

$$
\widetilde{\psi}_{u, v}^{\prime}(\tau)=-\frac{\sigma^{2}}{2} \widetilde{\psi}_{u, v}(\tau)^{2}-\frac{\delta^{\alpha}}{\alpha}\left(-\widetilde{\psi}_{u, v}(\tau)\right)^{\alpha}+b \widetilde{\psi}_{u, v}(\tau), \quad \tau \leqslant 0
$$

where $\widetilde{\psi}_{u, v}(0)=u, \quad \widetilde{\psi}_{u, v}\left(\tau_{*}\right)=0$, and, by the uniqueness of a locally bounded solution of the differential equation above, $\widetilde{\psi}_{u, v}(\tau)=0$ for $\tau \in\left[\tau_{*}, 0\right]$, i.e., $\psi_{u, v}(-\tau)=0$ for $\tau \in\left[-t_{*}, 0\right]$, i.e., $\psi_{u, v}(t)=0$ for $t \in\left[0, t_{*}\right]$. This yields us to a contradiction taking into account the definition of $t_{*}$ and the fact that $t_{*}>0$.

3.2 Remark. (i) By (iii) of Proposition 2.1, $\psi_{u, 0}(t)=-v_{t}(-u)$ for all $t \in \mathbb{R}_{+}$and $u \in \mathbb{R}_{-}$.

(ii) The differential equation (3.1) is a special case of Polyanin and Zaitsev [34, Section 1.5.1-2/(27)].

\section{Existence and uniqueness of MLE}

In this section, we will consider the stable CIR model (1.1) with known $a \in \mathbb{R}_{+}, \quad \sigma, \delta \in \mathbb{R}_{++}$, $\alpha \in(1,2)$, and a known deterministic initial value $Y_{0}=y_{0} \in \mathbb{R}_{+}$, and we will consider $b \in \mathbb{R}$ as an unknown parameter.

Let $\mathbb{P}_{b}$ denote the probability measure induced by $\left(Y_{t}\right)_{t \in \mathbb{R}_{+}}$on the measurable space $\left(D\left(\mathbb{R}_{+}, \mathbb{R}\right), \mathcal{D}\left(\mathbb{R}_{+}, \mathbb{R}\right)\right)$ endowed with the natural filtration $\left(\mathcal{D}_{t}\left(\mathbb{R}_{+}, \mathbb{R}\right)\right)_{t \in \mathbb{R}_{+}}$, see Appendix $\mathbb{A}$. Further, for all $T \in \mathbb{R}_{++}$, let $\mathbb{P}_{b, T}:=\left.\mathbb{P}_{b}\right|_{\mathcal{D}_{T}\left(\mathbb{R}_{+}, \mathbb{R}\right)}$ be the restriction of $\mathbb{P}_{b}$ to $\mathcal{D}_{T}\left(\mathbb{R}_{+}, \mathbb{R}\right)$.

The next result is about the form of the Radon-Nikodym derivative $\frac{\mathrm{d} \mathbb{P}_{b, T}}{\mathrm{~d} \mathbb{P}_{\widetilde{b}, T}}$ for $b, \widetilde{b} \in \mathbb{R}$. We will consider $\mathbb{P}_{\widetilde{b}, T}$ as a fixed reference measure, and we will derive the MLE for the parameter $b$ based on the observations $\left(Y_{t}\right)_{t \in[0, T]}$.

4.1 Proposition. Let $a \in \mathbb{R}_{+}, \quad b, \widetilde{b} \in \mathbb{R}, \sigma, \delta \in \mathbb{R}_{++}$, and $\alpha \in(1,2)$. Then for all $T \in \mathbb{R}_{++}$, the probability measures $\mathbb{P}_{b, T}$ and $\mathbb{P}_{\widetilde{b}, T}$ are absolutely continuous with respect to each other, and

$$
\log \left(\frac{\mathrm{d} \mathbb{P}_{b, T}}{\mathrm{~d} \mathbb{P}_{\widetilde{b}, T}}(\widetilde{Y})\right)=-\frac{b-\widetilde{b}}{\sigma^{2}}\left(\widetilde{Y}_{T}-y_{0}-a T-\delta \int_{0}^{T} \sqrt[\alpha]{\widetilde{Y}_{u-}} \mathrm{d} L_{u}\right)-\frac{b^{2}-\widetilde{b}^{2}}{2 \sigma^{2}} \int_{0}^{T} \widetilde{Y}_{u} \mathrm{~d} u
$$

holds $\mathbb{P}$-almost surely, where $\widetilde{Y}$ is the $\alpha$-stable CIR process corresponding to the parameter $\widetilde{b}$.

Proof. In what follows, we will apply Theorem III.5.34 in Jacod and Shiryaev [18] (see also Appendix A). We will work on the canonical space $\left(D\left(\mathbb{R}_{+}, \mathbb{R}\right), \mathcal{D}\left(\mathbb{R}_{+}, \mathbb{R}\right)\right)$. Let $\left(\eta_{t}\right)_{t \in \mathbb{R}_{+}}$denote the canonical process $\eta_{t}(\omega):=\omega(t), \omega \in D\left(\mathbb{R}_{+}, \mathbb{R}\right), t \in \mathbb{R}_{+}$. Recall that the stable CIR process (1.1) can be written in the form (2.8). By Proposition 2.1, the SDE (1.1) has a pathwise unique strong solution (with the given deterministic initial value $y_{0} \in \mathbb{R}_{+}$), and hence, by Theorem III.2.26 in Jacod and Shiryaev [18], under the probability measure $\mathbb{P}_{b}$, the canonical process $\left(\eta_{t}\right)_{t \in \mathbb{R}_{+}}$is a semimartingale with semimartingale characteristics $\left(B^{(b)}, C, \nu\right)$ associated with the truncation function $h$ (introduced in 
Proposition 2.2), where

$$
\begin{aligned}
& B_{t}^{(b)}=\int_{0}^{t}\left(a-b \eta_{u}+\gamma \delta \sqrt[\alpha]{\eta_{u}}+\int_{\mathbb{R}}\left(h\left(z \delta \sqrt[\alpha]{\eta_{u}}\right)-h(z) \delta \sqrt[\alpha]{\eta_{u}}\right) m(\mathrm{~d} z)\right) \mathrm{d} u, \quad t \in \mathbb{R}_{+}, \\
& C_{t}=\int_{0}^{t}\left(\sigma \sqrt{\eta_{u}}\right)^{2} \mathrm{~d} u=\sigma^{2} \int_{0}^{t} \eta_{u} \mathrm{~d} u, \quad t \in \mathbb{R}_{+}, \\
& \nu(\mathrm{d} t, \mathrm{~d} y)=K\left(\eta_{t}, \mathrm{~d} y\right) \mathrm{d} t
\end{aligned}
$$

with the Borel transition kernel $K$ from $\mathbb{R}_{+} \times \mathbb{R}$ into $\mathbb{R}$ given by

$$
K(y, R):=\int_{\mathbb{R}} \mathbb{1}_{R \backslash\{0\}}(z \delta \sqrt[\alpha]{y}) m(\mathrm{~d} z) \quad \text { for } \quad y \in \mathbb{R}_{+} \quad \text { and } \quad R \in \mathcal{B}(\mathbb{R})
$$

with $m(\mathrm{~d} z)=C_{\alpha} z^{-1-\alpha} \mathbb{1}_{(0, \infty)}(z) \mathrm{d} z$. The aim of the following discussion is to check the set of sufficient conditions presented in Appendix A (of which the notations will be used) in order to have right to apply Theorem III.5.34 in Jacod and Shiryaev [18]. First note that $\left(C_{t}\right)_{t \in \mathbb{R}_{+}}$and $\nu(\mathrm{d} t, \mathrm{~d} y)$ do not depend on the unknown parameter $b$, and hence $V^{(\widetilde{b}, b)}$ is identically one and then (A.1) and (A.2) readily hold. We also have

$$
\mathbb{P}_{b}(\nu(\{t\} \times \mathbb{R})=0)=\mathbb{P}_{b}\left(\int_{\{t\}} K\left(\eta_{s}, \mathbb{R}\right) \mathrm{d} s=0\right)=1, \quad t \in \mathbb{R}_{+}, \quad b \in \mathbb{R} .
$$

Further, $\left(C_{t}\right)_{t \in \mathbb{R}_{+}}$can be represented as $C_{t}=\int_{0}^{t} c_{u} \mathrm{~d} F_{u}, t \in \mathbb{R}_{+}$, where the stochastic processes $\left(c_{t}\right)_{t \in \mathbb{R}_{+}}$and $\left(F_{t}\right)_{t \in \mathbb{R}_{+}}$are given by $c_{t}:=\sigma^{2} \eta_{t}, t \in \mathbb{R}_{+}$, and $F_{t}=t, t \in \mathbb{R}_{+}$. Consequently, for all $b, \widetilde{b} \in \mathbb{R}$

$$
B_{t}^{(b)}-B_{t}^{(\widetilde{b})}=-(b-\widetilde{b}) \int_{0}^{t} \eta_{u} \mathrm{~d} u=\int_{0}^{t} c_{u} \beta_{u}^{(\widetilde{b}, b)} \mathrm{d} F_{u}
$$

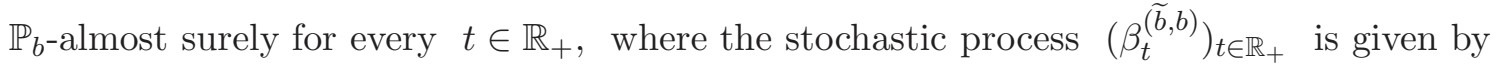

$$
\beta_{t}^{(\widetilde{b}, b)}=-\frac{b-\widetilde{b}}{\sigma^{2}}, \quad t \in \mathbb{R}_{+}
$$

which yields (A.3). Next we check A.4 i.e.,

$$
\mathbb{P}_{b}\left(\int_{0}^{t}\left(\beta_{u}^{(\widetilde{b}, b)}\right)^{2} c_{u} \mathrm{~d} F_{u}<\infty\right)=1, \quad t \in \mathbb{R}_{+}
$$

We have

$$
\int_{0}^{t}\left(\beta_{u}^{(\widetilde{b}, b)}\right)^{2} c_{u} \mathrm{~d} F_{u}=\frac{(b-\widetilde{b})^{2}}{\sigma^{2}} \int_{0}^{t} \eta_{u} \mathrm{~d} u, \quad t \in \mathbb{R}_{+} .
$$

Since for each $\omega \in D\left(\mathbb{R}_{+}, \mathbb{R}\right)$, the trajectory $[0, t] \ni u \mapsto \eta_{u}(\omega)$ is càdlàg, hence bounded (see, e.g., Billingsley [7, (12.5)]), we have $\int_{0}^{t} \eta_{u}(\omega) \mathrm{d} u<\infty$, hence we obtain (4.2).

Next, we check that, under the probability measure $\mathbb{P}_{b}$, local uniqueness holds for the martingale problem on the canonical space corresponding to the triplet $\left(B^{(b)}, C, \nu\right)$ with the given initial value $y_{0}$ with $\mathbb{P}_{b}$ as its unique solution (for the definition of local uniqueness in question, see Definition III.2.27 in Jacod and Shiryaev [18]). By Proposition 2.1, the SDE (1.1) has a pathwise unique strong 
solution (with the given deterministic initial value $y_{0} \in \mathbb{R}_{+}$), and hence Theorem III.2.26 in Jacod and Shiryaev [18] yields that the set of all solutions to the martingale problem on the canonical space corresponding to $\left(B^{(b)}, C, \nu\right)$ has only one element $\left(\mathbb{P}_{b}\right)$ yielding the desired local uniqueness. We also mention that Theorem III.4.29 in Jacod and Shiryaev [18] implies that under the probability measure $\mathbb{P}_{b}$, all local martingales have the integral representation property relative to $\eta$.

By Theorem III.5.34 in Jacod and Shiryaev [18] (see also Appendix [A), $\mathbb{P}_{b, T}$ and $\mathbb{P}_{\widetilde{b}, T}$ are equivalent (one can change the roles of $b$ and $\widetilde{b}$ ), and we have

$$
\frac{\mathrm{d} \mathbb{P}_{b, T}}{\mathrm{~d} \mathbb{P}_{\widetilde{b}, T}}(\eta)=\exp \left\{\int_{0}^{T} \beta_{u}^{(\widetilde{b}, b)} \mathrm{d}\left(\eta^{\text {cont }}\right)_{u}^{(\widetilde{b})}-\frac{1}{2} \int_{0}^{T}\left(\beta_{u}^{(\widetilde{b}, b)}\right)^{2} c_{u} \mathrm{~d} u\right\}, \quad T \in \mathbb{R}_{++}
$$

holds $\mathbb{P}_{\widetilde{b}}$-almost surely, where $\left(\left(\eta^{\text {cont }}\right)_{t}^{(\widetilde{b})}\right)_{t \in \mathbb{R}_{+}}$denotes the continuous (local) martingale part of $\left(\eta_{t}\right)_{t \in \mathbb{R}_{+}}$under $\mathbb{P}_{\widetilde{b}}$. Using Remarks III.2.28 in Jacod and Shiryaev [18] and (2.8), the continuous (local) martingale part $\left(\widetilde{Y}_{t}^{\text {cont }}\right)_{t \in \mathbb{R}_{+}}$of $\left(\widetilde{Y}_{t}\right)_{t \in \mathbb{R}_{+}}$takes the form $\tilde{Y}_{t}^{\text {cont }}=\sigma \int_{0}^{t} \sqrt{\widetilde{Y}_{u}} \mathrm{~d} W_{u}, \quad t \in \mathbb{R}_{+}$, and, by (1.1), we have

$$
\mathrm{d} \widetilde{Y}_{t}^{\text {cont }}=\mathrm{d} \widetilde{Y}_{t}-\left(a-\widetilde{b} \widetilde{Y}_{t}\right) \mathrm{d} t-\delta \sqrt[\alpha]{\widetilde{Y}_{t-}} \mathrm{d} L_{t}, \quad t \in \mathbb{R}_{+} .
$$

Hence

$$
\begin{aligned}
\log \left(\frac{\mathrm{d} \mathbb{P}_{b, T}}{\mathrm{~d} \mathbb{P}_{\widetilde{b}, T}}(\widetilde{Y})\right)= & \int_{0}^{T}\left(-\frac{b-\widetilde{b}}{\sigma^{2}}\right)\left(\mathrm{d} \widetilde{Y}_{u}-\delta \sqrt[\alpha]{\widetilde{Y}_{u-}} \mathrm{d} L_{u}\right)-\int_{0}^{T}\left(-\frac{b-\widetilde{b}}{\sigma^{2}}\right)\left(a-\widetilde{b}_{u}\right) \mathrm{d} u \\
& -\frac{1}{2} \int_{0}^{T}\left(-\frac{b-\widetilde{b}}{\sigma^{2}}\right)^{2} \sigma^{2} \widetilde{Y}_{u} \mathrm{~d} u \\
= & -\frac{b-\widetilde{b}}{\sigma^{2}} \int_{0}^{T}\left(\mathrm{~d} \widetilde{Y}_{u}-\delta \sqrt[\alpha]{\widetilde{Y}_{u-}} \mathrm{d} L_{u}\right)+\frac{b-\widetilde{b}}{\sigma^{2}} \int_{0}^{T} a \mathrm{~d} u-\frac{b^{2}-\widetilde{b}^{2}}{2 \sigma^{2}} \int_{0}^{T} \widetilde{Y}_{u} \mathrm{~d} u
\end{aligned}
$$

holds $\mathbb{P}$-almost surely, which yields the statement.

Next, using Proposition 4.1, by considering $\mathbb{P}_{\widetilde{b}, T}$ as a fixed reference measure, we derive an MLE for the parameter $b$ based on the observations $\left(Y_{t}\right)_{t \in[0, T]}$. Let us denote the right hand side of (4.1) by $\Lambda_{T}(b, \widetilde{b})$ replacing $\widetilde{Y}$ by $Y$. By an MLE $\widehat{b}_{T}$ of the parameter $b$ based on the observations $\left(Y_{t}\right)_{t \in[0, T]}$, we mean

$$
\widehat{b}_{T}:=\underset{b \in \mathbb{R}}{\arg \max } \Lambda_{T}(b, \widetilde{b}),
$$

which will turn out to be not dependent on $\widetilde{b}$. Our method for deriving an MLE is one of the known ones in the literature, and it turns out that these lead to the same estimator $\widehat{b}_{T}$, see Remark 4.4 .

Next, we formulate a result about the unique existence of MLE $\widehat{b}_{T}$ of $b$ for all $T \in \mathbb{R}_{++}$.

4.2 Proposition. Let $a \in \mathbb{R}_{+}, \quad b \in \mathbb{R}, \quad \sigma, \delta \in \mathbb{R}_{++}, \quad \alpha \in(1,2)$, and $y_{0} \in \mathbb{R}_{+}$. If $a \in \mathbb{R}_{++}$or $y_{0} \in \mathbb{R}_{++}$, then for each $T \in \mathbb{R}_{++}$, there exists a unique $M L E \widehat{b}_{T}$ of $b \mathbb{P}$-almost surely having the form

$$
\widehat{b}_{T}=-\frac{Y_{T}-y_{0}-a T-\delta \int_{0}^{T} \sqrt[\alpha]{Y_{u-}} \mathrm{d} L_{u}}{\int_{0}^{T} Y_{s} \mathrm{~d} s}
$$

provided that $\int_{0}^{T} Y_{s} \mathrm{~d} s \in \mathbb{R}_{++}$(which holds $\mathbb{P}$-almost surely due to (v) of Proposition (2.1). 
Proof. Due to (v) of Proposition 2.1, $\mathbb{P}\left(\int_{0}^{T} Y_{s} \mathrm{~d} s \in \mathbb{R}_{++}\right)=1$ for all $T \in \mathbb{R}_{++}$, and hence the right hand side of (4.3) is well-defined $\mathbb{P}$-almost surely. The aim of the following discussion is to show that the right hand side of (4.3) is a measurable function of $\left(Y_{u}\right)_{u \in[0, T]}$ (i.e., a statistic). By Proposition 4.1 , for all $b, \widetilde{b} \in \mathbb{R}$ and $T \in \mathbb{R}_{++}$, the probability measures $\mathbb{P}_{\widetilde{b}, T}$ and $\mathbb{P}_{b, T}$ are absolutely continuous with respect to each other, and

$$
\log \left(\frac{\mathrm{d} \mathbb{P}_{\widetilde{b}, T}}{\mathrm{~d} \mathbb{P}_{b, T}}(Y)\right)=-\frac{\widetilde{b}-b}{\sigma^{2}}\left(Y_{T}-y_{0}-a T-\delta \int_{0}^{T} \sqrt[\alpha]{Y_{u-}} \mathrm{d} L_{u}\right)-\frac{\widetilde{b}^{2}-b^{2}}{2 \sigma^{2}} \int_{0}^{T} Y_{u} \mathrm{~d} u
$$

holds $\mathbb{P}$-almost surely.

The left-hand side of the above equality is measurable with respect to $\left(Y_{t}\right)_{t \in[0, T]}$ (see, e.g., Jacod and Shiryaev [18, Theorem III.3.4]), and hence its right hand-side is also measurable, which yields the measurability of $\int_{0}^{T} \sqrt[\alpha]{Y_{u-}} \mathrm{d} L_{u}$ with respect to $\left(Y_{t}\right)_{t \in[0, T]}$ and consequently that of $\widehat{b}_{T}$.

By Proposition 4.1, for all $b, \widetilde{b} \in \mathbb{R}$, we have

$$
\begin{aligned}
& \frac{\partial}{\partial b} \log \left(\Lambda_{T}(b, \widetilde{b})\right)=-\frac{1}{\sigma^{2}}\left(Y_{T}-y_{0}-a T-\delta \int_{0}^{T} \sqrt[\alpha]{Y_{u-}} \mathrm{d} L_{u}\right)-\frac{b}{\sigma^{2}} \int_{0}^{T} Y_{u} \mathrm{~d} u \\
& \frac{\partial^{2}}{\partial b^{2}} \log \left(\Lambda_{T}(b, \widetilde{b})\right)=-\frac{1}{\sigma^{2}} \int_{0}^{T} Y_{u} \mathrm{~d} u
\end{aligned}
$$

Thus the MLE $\widehat{b}_{T}$ of $b$ based on a continuous time observation $\left(Y_{s}\right)_{s \in[0, T]}$ exists $\mathbb{P}$-almost surely, and it takes the form (4.3) provided that $\int_{0}^{T} Y_{s} \mathrm{~d} s \in \mathbb{R}_{++}$.

4.3 Remark. In what follows, under the assumptions of Proposition 4.2 and the additional assumption $a \geqslant \frac{\sigma^{2}}{2}$, we prove that $L_{t}$ is a measurable function of $\left(Y_{u}\right)_{u \in[0, T]}$ for all $t \in[0, T]$, where $T \in \mathbb{R}_{++}$, which (in the special case $a \geqslant \frac{\sigma^{2}}{2}$ ) gives another proof for the fact that the right hand side of (4.3) is a statistic. Recalling the notation $\zeta_{t}=\delta \int_{0}^{t} \sqrt[\alpha]{Y_{u-}} \mathrm{d} L_{u}, t \in \mathbb{R}_{+}$, we have $\Delta \zeta_{t}=\delta \sqrt[\alpha]{Y_{t-}} \Delta L_{t}$, $t \in \mathbb{R}_{+}$(following from (2.1) and Jacod and Shiryaev [18, Definitions II.1.27]) and using the SDE (1.1), we get $\Delta Y_{t}=\Delta \zeta_{t}=\delta \sqrt[\alpha]{Y_{t-}} \Delta L_{t}, t \in \mathbb{R}_{+}$. Hence $\Delta L_{t}=\frac{\Delta Y_{t}}{\delta \sqrt[\alpha]{Y_{t-}}}, t \in \mathbb{R}_{++}$, since, by (iv) of Proposition 2.1. $\mathbb{P}\left(Y_{t} \in \mathbb{R}_{++}\right.$for all $\left.t \in \mathbb{R}_{++}\right)=1$. For all $t \in[0, T]$ and $\varepsilon \in(0,1)$,

$$
\begin{aligned}
& \int_{(0, t]} \int_{\{\varepsilon<|z| \leqslant 1\}} z \widetilde{\mu}^{L}(\mathrm{~d} u, \mathrm{~d} z)=\sum_{u \in[0, t]} \mathbb{1}_{\left\{\varepsilon<\left|\Delta L_{u}\right| \leqslant 1\right\}} \Delta L_{u}-\int_{(0, t]} \int_{\{\varepsilon<|z| \leqslant 1\}} z \mathrm{~d} u m(\mathrm{~d} z) \\
& =\sum_{u \in[0, t]} \mathbb{1}_{\left\{\varepsilon<\frac{\left|\Delta Y_{u}\right|}{\delta \sqrt[\alpha]{Y_{u-}}} \leqslant 1\right\}} \frac{\Delta Y_{u}}{\delta \sqrt[\alpha]{Y_{u-}}}-t \int_{\{\varepsilon<|z| \leqslant 1\}} z m(\mathrm{~d} z),
\end{aligned}
$$

which is a measurable function of $\left(Y_{t}\right)_{t \in[0, T]}$. Similarly, for all $t \in[0, T]$,

$$
\int_{(0, t]} \int_{\{|z|>1\}} z \mu^{L}(\mathrm{~d} u, \mathrm{~d} z)=\sum_{u \in[0, t]} \mathbb{1}_{\left\{\left|\Delta L_{u}\right|>1\right\}} \Delta L_{u}=\sum_{u \in[0, t]} \mathbb{1}_{\left\{\frac{\left|\Delta Y_{u}\right|}{\delta \sqrt[\alpha]{Y_{u-}}}>1\right\}} \frac{\Delta Y_{u}}{\delta \sqrt[\alpha]{Y_{u-}}},
$$

which is a measurable function of $\left(Y_{u}\right)_{u \in[0, T]}$ as well. Hence, using (2.1), for all $t \in[0, T]$,

$$
\sum_{u \in[0, t]} \mathbb{1}_{\left\{\frac{\left|\Delta Y_{u}\right|}{\delta \sqrt[\alpha]{Y_{u-}}}>\varepsilon\right\}} \frac{\Delta Y_{u}}{\delta \sqrt[\alpha]{Y_{u-}}}-t \int_{\{\varepsilon<|z| \leqslant 1\}} z m(\mathrm{~d} z)+\gamma t \stackrel{\mathbb{P}}{\longrightarrow} L_{t} \quad \text { as } \quad \varepsilon \downarrow 0,
$$


yielding that $L_{t}$ is a measurable function of $\left(Y_{t}\right)_{t \in[0, T]}$. Finally, note also that if $Y_{t-}=0, t \in \mathbb{R}_{+}$, then, using $\Delta Y_{t}=\delta \sqrt[\alpha]{Y_{t-}} \Delta L_{t}, t \in \mathbb{R}_{+}$, we have $\Delta Y_{t}=0$ yielding $Y_{t}=Y_{t-}=0$ (irrespective of the size of the jump of $L$ at $t$ ).

4.4 Remark. In the literature there is another way of deriving an MLE. Sørensen [36] defined an MLE of $b$ as a solution of the equation $\dot{\Lambda}_{T}(b)=0$, where $\dot{\Lambda}_{T}(b)$ is the so-called score vector given in formula (3.3) in Sørensen [36]. Luschgy [29, [30] called this equation as an estimating equation. With the notations of the proof of Proposition 4.1, taking into account of the form of $\beta^{(\widetilde{b}, b)}$ and the fact that $V^{(\widetilde{b}, b)}$ is identically one, we have

$$
\begin{aligned}
\dot{\Lambda}_{T}(b) & :=\int_{0}^{T}\left(-\frac{1}{\sigma^{2}}\right) \mathrm{d} Y_{u}^{\mathrm{cont}}=-\frac{1}{\sigma^{2}} \int_{0}^{T}\left(\mathrm{~d} Y_{u}-\left(a-b Y_{u}\right) \mathrm{d} u-\delta \sqrt[\alpha]{Y_{u-}} \mathrm{d} L_{u}\right) \\
& =-\frac{1}{\sigma^{2}}\left(Y_{T}-y_{0}-a T+b \int_{0}^{T} Y_{u} \mathrm{~d} u-\delta \int_{0}^{T} \sqrt[\alpha]{Y_{u-}} \mathrm{d} L_{u}\right)
\end{aligned}
$$

for $b \in \mathbb{R}$ and $T \in \mathbb{R}_{++}$. The estimating equation $\dot{\Lambda}_{T}(b)=0, \quad b \in \mathbb{R}$, has a unique solution $-\frac{Y_{T}-y_{0}-a T-\delta \int_{0}^{T} \sqrt[\alpha]{Y_{u-}} \mathrm{d} L_{u}}{\int_{0}^{T} Y_{u} \mathrm{~d} u}$ provided that $\int_{0}^{T} Y_{u} \mathrm{~d} u$ is strictly positive, which holds $\mathbb{P}$-almost surely provided that $a \in \mathbb{R}_{++}$or $y_{0} \in \mathbb{R}_{++}$(due to (v) of Proposition 2.1). Recall that this unique solution coincides with $\widehat{b}_{T}$, see (4.3).

\section{Asymptotic behavior of the MLE in the subcritical case}

If $a \in \mathbb{R}_{++}$or $y_{0} \in \mathbb{R}_{++}$, then, using (4.3) and the SDE (1.1), we get

$$
\widehat{b}_{T}-b=-\frac{Y_{T}-y_{0}-a T-\delta \int_{0}^{T} \sqrt[\alpha]{Y_{u-}} \mathrm{d} L_{u}+b \int_{0}^{T} Y_{s} \mathrm{~d} s}{\int_{0}^{T} Y_{s} \mathrm{~d} s}=-\frac{\sigma \int_{0}^{T} \sqrt{Y_{s}} \mathrm{~d} W_{s}}{\int_{0}^{T} Y_{s} \mathrm{~d} s}
$$

provided that $\int_{0}^{T} Y_{s} \mathrm{~d} s \in \mathbb{R}_{++}$, which holds $\mathbb{P}$-almost surely due to (v) of Proposition 2.1. Here note that $\sigma \int_{0}^{T} \sqrt{Y_{s}} \mathrm{~d} W_{s}=Y_{T}^{\text {cont }}, T \in \mathbb{R}_{+}$, due to part 1) of Remarks III.2.28 in Jacod and Shyriaev [18] and (2.8).

5.1 Theorem. Let $a, b, \sigma, \delta \in \mathbb{R}_{++}$and $\alpha \in(1,2)$. Let $\left(Y_{t}\right)_{t \in \mathbb{R}_{+}}$be the unique strong solution of the SDE (1.1) satisfying $\mathbb{P}\left(Y_{0}=y_{0}\right)=1$ with some $y_{0} \in \mathbb{R}_{+}$. Then the MLE $\widehat{b}_{T}$ of $b$ is strongly consistent and asymptotically normal, i.e., $\widehat{b}_{T} \stackrel{\text { a.s. }}{\longrightarrow} b$ as $T \rightarrow \infty$, and

$$
\sqrt{T}\left(\widehat{b}_{T}-b\right) \stackrel{\mathcal{D}}{\longrightarrow} \mathcal{N}\left(0, \frac{\sigma^{2} b}{a}\right) \quad \text { as } T \rightarrow \infty .
$$

With a random scaling,

$$
\frac{1}{\sigma}\left(\int_{0}^{T} Y_{s} \mathrm{~d} s\right)^{1 / 2}\left(\widehat{b}_{T}-b\right) \stackrel{\mathcal{D}}{\longrightarrow} \mathcal{N}(0,1) \quad \text { as } T \rightarrow \infty .
$$

Proof. By Proposition 4.2, there exists a unique MLE $\widehat{b}_{T}$ of $b$ for all $T \in \mathbb{R}_{++}$, which has the form given in (4.3). By (i) of Theorem 2.5. $\left(Y_{t}\right)_{t \in \mathbb{R}_{+}}$has a unique stationary distribution $\pi$ with $\int_{0}^{\infty} y \pi(\mathrm{d} y)=\frac{a}{b} \in \mathbb{R}_{++}$. By (ii) of Theorem 2.5. we have $\frac{1}{T} \int_{0}^{T} Y_{s} \mathrm{~d} s \stackrel{\text { a.s. }}{\longrightarrow} \int_{0}^{\infty} y \pi(\mathrm{d} y)$ as $T \rightarrow \infty$, 
implying also $\int_{0}^{T} Y_{s} \mathrm{~d} s \stackrel{\text { a.s. }}{\longrightarrow} \infty$ as $T \rightarrow \infty$. Since the quadratic variation process of the square integrable martingale $\left(\int_{0}^{t} \sqrt{Y_{s}} \mathrm{~d} W_{s}\right)_{t \in \mathbb{R}_{+}}$takes the form $\left(\int_{0}^{t} Y_{s} \mathrm{~d} s\right)_{t \in \mathbb{R}_{+}}$, using (5.1) and Theorem B.1, we have $\widehat{b}_{T} \stackrel{\text { a.s. }}{\longrightarrow} b$ as $T \rightarrow \infty$. Moreover, using Theorem B.2 with $\eta:=\left(\int_{0}^{\infty} y \pi(\mathrm{d} y)\right)^{1 / 2}$ and Slutsky's lemma, we have

$$
\sqrt{T}\left(\widehat{b}_{T}-b\right)=-\sigma \frac{\frac{1}{\sqrt{T}} \int_{0}^{T} \sqrt{Y_{s}} \mathrm{~d} W_{s}}{\frac{1}{T} \int_{0}^{T} Y_{s} \mathrm{~d} s} \stackrel{\mathcal{D}}{\longrightarrow}-\sigma \frac{\left(\int_{0}^{\infty} y \pi(\mathrm{d} y)\right)^{1 / 2} \mathcal{N}(0,1)}{\int_{0}^{\infty} y \pi(\mathrm{d} y)}=\mathcal{N}\left(0, \frac{\sigma^{2}}{\int_{0}^{\infty} y \pi(\mathrm{d} y)}\right)
$$

as $T \rightarrow \infty$, hence, by (2.16), we obtain (5.2). Further, Slutsky's lemma yields

$$
\begin{aligned}
\frac{1}{\sigma}\left(\int_{0}^{T} Y_{s} \mathrm{~d} s\right)^{1 / 2}\left(\widehat{b}_{T}-b\right) & =\frac{1}{\sigma}\left(\frac{1}{T} \int_{0}^{T} Y_{s} \mathrm{~d} s\right)^{1 / 2} \sqrt{T}\left(\widehat{b}_{T}-b\right) \\
& \stackrel{\mathcal{D}}{\longrightarrow} \frac{1}{\sigma}\left(\int_{0}^{\infty} y \pi(\mathrm{d} y)\right)^{1 / 2} \mathcal{N}\left(0, \frac{\sigma^{2}}{\int_{0}^{\infty} y \pi(\mathrm{d} y)}\right)=\mathcal{N}(0,1)
\end{aligned}
$$

as $T \rightarrow \infty$.

\section{Consistency of the MLE in the critical case}

First, we describe the asymptotic behavior of the solution $\psi_{u, v}$ of the differential equation (3.1) as $t \rightarrow \infty$ in case of $b=0$ using a so-called separator technique.

Note that, for $b, \sigma \in \mathbb{R}_{+}, \quad \delta \in \mathbb{R}_{++}$and $\alpha \in(1,2)$, the function $\mathbb{R}_{-} \ni x \mapsto \widetilde{R}(x):=R(-x)=$ $\frac{\sigma^{2}}{2} x^{2}+\frac{\delta^{\alpha}}{\alpha}(-x)^{\alpha}-b x$ is strictly monotone decreasing, continuous, convex and $\lim _{x \rightarrow-\infty} \widetilde{R}(x)=+\infty$. Indeed, $\widetilde{R}^{\prime}(x)=\sigma^{2} x-\delta^{\alpha}(-x)^{\alpha-1}-b<0, \quad x \in \mathbb{R}_{--}, \quad$ and $\widetilde{R}^{\prime \prime}(x)=\sigma^{2}+\delta^{\alpha}(\alpha-1)(-x)^{\alpha-2}>0$, $x \in \mathbb{R}_{--}$. Hence, for all $v \in \mathbb{R}_{--}$, the equation $R(-x)=\frac{\sigma^{2}}{2} x^{2}+\frac{\delta^{\alpha}}{\alpha}(-x)^{\alpha}-b x=-v, x \in \mathbb{R}_{-}$, has a unique negative solution, denoted by $c_{v} \in \mathbb{R}_{--}$. Further, if $x \in\left(c_{v}, 0\right]$, then $R(-x)<-v$, i.e., $R(-x)+v<0$; and if $x \in\left(-\infty, c_{v}\right)$, then $R(-x)>-v$, i.e., $R(-x)+v>0$.

6.1 Proposition. Let $a \in \mathbb{R}_{+}, \quad b=0, \quad \sigma \in \mathbb{R}_{+}, \quad \delta \in \mathbb{R}_{++}$, and $\alpha \in(1,2)$. Then for all $u \in \mathbb{R}_{-}$ and $v \in \mathbb{R}_{--}$, the unique locally bounded solution $\psi_{u, v}$ of the differential equation (3.1) satisfies $\lim _{t \rightarrow \infty} \psi_{u, v}(t)=c_{v}$. Further, $\psi_{u, v}(t) \in\left(c_{v}, 0\right], \quad t \in \mathbb{R}_{+} \quad$ if $c_{v}<u \leqslant 0 ; \psi_{u, v}(t) \in\left(-\infty, c_{v}\right), \quad t \in \mathbb{R}_{+}$ if $u<c_{v}$; and $\psi_{u, v}(t)=c_{v}, t \in \mathbb{R}_{+}$if $u=c_{v}$.

Proof. Let $v \in \mathbb{R}_{--}$be fixed. By Theorem 3.1 $\psi_{u, v}(t) \in \mathbb{R}_{--}$for all $t \in \mathbb{R}_{++}$. Let $Q(x):=$ $R(-x)+v=\frac{\sigma^{2}}{2} x^{2}+\frac{\delta^{\alpha}}{\alpha}(-x)^{\alpha}+v, \quad x \in \mathbb{R}_{-}$. Note that $Q$ is continuously differentiable on $\mathbb{R}_{--}$. Further, $Q(x)=0, x \in \mathbb{R}_{-}$, holds if and only if $x=c_{v}$, and $Q(x)<0$ for $x \in\left(c_{v}, 0\right]$ and $Q(x)>0$ for $x \in\left(-\infty, c_{v}\right)$.

If $\psi_{u, v}(0)=u=c_{v}$, then the unique locally bounded solution of the differential equation (3.1) takes the form $\psi_{u, v}(t)=c_{v}, \quad t \in \mathbb{R}_{+}$, since in this case $\psi_{u, v}^{\prime}(t)=0, \quad t \in \mathbb{R}_{+}$, and $\frac{\sigma^{2}}{2}\left(\psi_{u, v}(t)\right)^{2}+$ $\frac{\delta^{\alpha}}{\alpha}\left(-\psi_{u, v}(t)\right)^{\alpha}+v=\frac{\sigma^{2}}{2} c_{v}^{2}+\frac{\delta^{\alpha}}{\alpha}\left(-c_{v}\right)^{\alpha}+v=Q\left(c_{v}\right)=0, \quad t \in \mathbb{R}_{+}$, and hence (3.1) holds.

If $\psi_{u, v}(0)=u>c_{v}$, then $\psi_{u, v}(t)>c_{v}$ for all $t \in \mathbb{R}_{+}$. Indeed, on the contrary, let us suppose that there exists $t_{0} \in \mathbb{R}_{++}$such that $\psi_{u, v}\left(t_{0}\right)=c_{v}$ (which can be supposed due to the continuity of $\left.\psi_{u, v}\right)$. Then $\psi_{u, v}(t)=c_{v}$ would hold for all $t \in \mathbb{R}_{+}$, since it is known that if two maximal solutions 
of an autonomous ordinary differential equation (with a continuously differentiable function on the right hand side) coincide at some points, then their ranges coincide, see, e.g., Arnol'd [3, Corollary on page 118], and the identically $c_{v}$ function is a solution of (3.1) (without the initial value). Since $\psi_{u, v}(0)>c_{v}$, this leads us to a contradiction. Consequently, by (3.1), $\psi_{u, v}^{\prime}(t)=Q\left(\psi_{u, v}(t)\right)<0$, $t \in \mathbb{R}_{+}$, yielding that $\psi_{u, v}$ is monotone decreasing. Since $\psi_{u, v}$ is bounded below by $c_{v}$, there exists an $\widetilde{c}_{v} \in \mathbb{R}_{-}$such that $\widetilde{c}_{v} \geqslant c_{v}$ and $\lim _{t \rightarrow \infty} \psi_{u, v}(t)=\widetilde{c}_{v}$. It remains to check that $\widetilde{c}_{v}=c_{v}$. We show that $Q\left(\widetilde{c}_{v}\right)=0$, yielding $\widetilde{c}_{v}=c_{v}$, since $c_{v}$ is the only root of $Q(x)=0, x \in \mathbb{R}_{-}$. On the contrary, let us suppose that $Q\left(\widetilde{c}_{v}\right)>0$ (the case $Q\left(\widetilde{c}_{v}\right)<0$ can be handled similarly). Since $Q$ is continuous at $\widetilde{c}_{v}$, there exists $\kappa>0$ such that $Q(x)>\frac{Q\left(\widetilde{c}_{v}\right)}{2}$ for all $x \in \mathbb{R}_{-}$satisfying $\left|x-\widetilde{c}_{v}\right|<\kappa$. Since $\lim _{t \rightarrow \infty} \psi_{u, v}(t)=\widetilde{c}_{v}$, there exists $T>0$ such that $\left|\psi_{u, v}(t)-\widetilde{c}_{v}\right|<\kappa$ for $t \geqslant T$.

Hence

$$
\psi_{u, v}^{\prime}(t)=Q\left(\psi_{u, v}(t)\right)>\frac{Q\left(\widetilde{c}_{v}\right)}{2}, \quad t \geqslant T
$$

Integrating over $[T, t]$, we have

$$
\psi_{u, v}(t)-\psi_{u, v}(T) \geqslant \frac{Q\left(\widetilde{c}_{v}\right)}{2}(t-T), \quad t \geqslant T .
$$

Since, by assumption, $Q\left(\widetilde{c}_{v}\right)>0$, taking the limit $t \rightarrow \infty$, we get $\lim _{t \rightarrow \infty} \psi_{u, v}(t)=\infty$, yielding us to a contradiction.

The case $\psi_{u, v}(0)=u<c_{v}$ can be handled similarly, and the other parts of the proposition follow as well.

6.2 Theorem. Let $a \in \mathbb{R}_{++}, \quad b=0, \quad \sigma, \delta \in \mathbb{R}_{++}$, and $\alpha \in(1,2)$. Let $\left(Y_{t}\right)_{t \in \mathbb{R}_{+}}$be the unique strong solution of the $S D E(\mathbb{1 . 1})$ satisfying $\mathbb{P}\left(Y_{0}=y_{0}\right)=1$ with some $y_{0} \in \mathbb{R}_{+}$. Then the MLE of $b$ is strongly consistent, i.e., $\widehat{b}_{T} \stackrel{\text { a.s. }}{\longrightarrow} b$ as $T \rightarrow \infty$.

Proof. Since $\left(\int_{0}^{t} Y_{s} \mathrm{~d} s\right)_{t \in \mathbb{R}_{+}}$is monotone increasing $\mathbb{P}$-almost surely, there exists an $[0, \infty]$-valued random variable $\xi$ such that $\int_{0}^{t} Y_{s} \mathrm{~d} s \stackrel{\text { a.s. }}{\rightarrow} \xi$ as $t \rightarrow \infty$, and consequently, by the dominated convergence theorem, $\lim _{t \rightarrow \infty} \mathbb{E}\left[\exp \left\{v \int_{0}^{t} Y_{s} \mathrm{~d} s\right\}\right]=\mathbb{E}\left[\mathrm{e}^{v \xi}\right]$ for any $v \in \mathbb{R}_{-}$. By Theorem 3.1 with $b=0$, we have

$$
\mathbb{E}\left[\exp \left\{v \int_{0}^{t} Y_{s} \mathrm{~d} s\right\}\right]=\exp \left\{y_{0} \psi_{0, v}(t)+a \int_{0}^{t} \psi_{0, v}(s) \mathrm{d} s\right\}, \quad t \in \mathbb{R}_{+}, \quad v \in \mathbb{R}_{-} .
$$

First we check that

$$
\int_{0}^{t} \psi_{0, v}(s) \mathrm{d} s=\int_{0}^{\psi_{0, v}(t)} \frac{x}{\frac{\sigma^{2}}{2} x^{2}+\frac{\delta^{\alpha}}{\alpha}(-x)^{\alpha}+v} \mathrm{~d} x
$$

for all $t \in \mathbb{R}_{++}$and $v \in \mathbb{R}_{--}$, where the function $\psi_{0, v}: \mathbb{R}_{+} \rightarrow \mathbb{R}_{-}$is given by (3.1). Recall that, for all $v \in \mathbb{R}_{--}, c_{v} \in \mathbb{R}_{--}$denotes the unique negative solution of the equation $\frac{\sigma^{2}}{2} x^{2}+\frac{\delta^{\alpha}}{\alpha}(-x)^{\alpha}+v=0$, we have $\frac{\sigma^{2}}{2} x^{2}+\frac{\delta^{\alpha}}{\alpha}(-x)^{\alpha}+v<0$ for all $x \in\left(c_{v}, 0\right]$, and, by Proposition [6.1, $\psi_{0, v}(t) \in\left(c_{v}, 0\right]$ for all $t \in \mathbb{R}_{+}$. Consequently, by (3.1), the function $[0, t] \ni s \mapsto \psi_{0, v}(s) \in\left(c_{v}, 0\right]$ is strictly decreasing and continuously differentiable, hence, for all $t \in \mathbb{R}_{++}$, by the substitution $x=\psi_{0, v}(s)$ we obtain

$$
\int_{0}^{t} \psi_{0, v}(s) \mathrm{d} s=\int_{0}^{\psi_{0, v}(t)} \frac{x}{\psi_{0, v}^{\prime}\left(\psi_{0, v}^{-1}(x)\right)} \mathrm{d} x
$$


and hence (6.1), where $\psi_{0, v}^{-1}$ denotes the inverse of $\psi_{0, v}$. By (6.1), we have

$$
\mathbb{E}\left[\exp \left\{v \int_{0}^{t} Y_{s} \mathrm{~d} s\right\}\right]=\exp \left\{y_{0} \psi_{0, v}(t)+a \int_{0}^{\psi_{0, v}(t)} \frac{x}{\frac{\sigma^{2}}{2} x^{2}+\frac{\delta^{\alpha}}{\alpha}(-x)^{\alpha}+v} \mathrm{~d} x\right\}
$$

for $t \in \mathbb{R}_{++}$and $v \in \mathbb{R}_{--}$. Then, by Proposition 6.1, $\lim _{t \rightarrow \infty} \psi_{0, v}(t)=c_{v}$, and hence

$$
\lim _{t \rightarrow \infty} \int_{0}^{\psi_{0, v}(t)} \frac{x}{\frac{\sigma^{2}}{2} x^{2}+\frac{\delta^{\alpha}}{\alpha}(-x)^{\alpha}+v} \mathrm{~d} x=\int_{0}^{c_{v}} \frac{x}{\frac{\sigma^{2}}{2} x^{2}+\frac{\delta^{\alpha}}{\alpha}(-x)^{\alpha}+v} \mathrm{~d} x=-\infty .
$$

Here the last step can be checked as follows. We have

$$
\lim _{x \rightarrow c_{v}} \frac{\frac{\sigma^{2}}{2} x^{2}+\frac{\delta^{\alpha}}{\alpha}(-x)^{\alpha}+v}{x-c_{v}}=\lim _{x \rightarrow c_{v}} \frac{(R(-x)+v)-\left(R\left(-c_{v}\right)+v\right)}{x-c_{v}}=-R^{\prime}\left(-c_{v}\right)=\sigma^{2} c_{v}-\delta^{\alpha}\left(-c_{v}\right)^{\alpha-1}<0,
$$

thus there exists $x_{0} \in\left(c_{v}, 0\right)$ such that $\frac{\frac{\sigma^{2}}{2} x^{2}+\frac{\delta^{\alpha}}{\alpha}(-x)^{\alpha}+v}{x-c_{v}} \leqslant \frac{\sigma^{2} c_{v}-\delta^{\alpha}\left(-c_{v}\right)^{\alpha-1}}{2}$ for all $x \in\left(c_{v}, x_{0}\right)$. Hence

$$
\begin{aligned}
\int_{0}^{c_{v}} \frac{x}{\frac{\sigma^{2}}{2} x^{2}+\frac{\delta^{\alpha}}{\alpha}(-x)^{\alpha}+v} \mathrm{~d} x & \leqslant \int_{x_{0}}^{c_{v}} \frac{x}{\frac{\sigma^{2}}{2} x^{2}+\frac{\delta^{\alpha}}{\alpha}(-x)^{\alpha}+v} \mathrm{~d} x \\
& \leqslant \frac{2 x_{0}}{\sigma^{2} c_{v}-\delta^{\alpha}\left(-c_{v}\right)^{\alpha-1}} \int_{x_{0}}^{c_{v}} \frac{1}{x-c_{v}} \mathrm{~d} x=-\infty
\end{aligned}
$$

as desired. Hence, since $a \in \mathbb{R}_{++}$, we have

$$
\lim _{t \rightarrow \infty} \mathbb{E}\left[\exp \left\{v \int_{0}^{t} Y_{s} \mathrm{~d} s\right\}\right]=\exp \left\{y_{0} c_{v}+a(-\infty)\right\}=0, \quad v \in \mathbb{R}_{--},
$$

which yields that $\mathbb{E}\left(\mathrm{e}^{v \xi}\right)=0, v \in \mathbb{R}_{--}$. Since, for all $v \in \mathbb{R}_{--}$,

$$
\begin{aligned}
\mathbb{E}\left(\mathrm{e}^{v \xi}\right) & =\mathbb{E}\left(\mathrm{e}^{v \xi} \mid \xi=\infty\right) \mathbb{P}(\xi=\infty)+\mathbb{E}\left(\mathrm{e}^{v \xi} \mid \xi<\infty\right) \mathbb{P}(\xi<\infty) \\
& =0 \cdot \mathbb{P}(\xi=\infty)+\mathbb{E}\left(\mathrm{e}^{v \xi} \mid \xi<\infty\right) \mathbb{P}(\xi<\infty),
\end{aligned}
$$

we have $0=\mathbb{E}\left(\mathrm{e}^{v \xi} \mid \xi<\infty\right) \mathbb{P}(\xi<\infty)$, yielding that $\mathbb{P}(\xi<\infty)=0$, i.e., $\mathbb{P}(\xi=\infty)=1$. That is, we have proved that $\int_{0}^{t} Y_{s} \mathrm{~d} s \stackrel{\text { a.s. }}{\longrightarrow} \infty$ as $t \rightarrow \infty$. Since the quadratic variation process of the square integrable martingale $\left(\int_{0}^{t} \sqrt{Y_{s}} \mathrm{~d} W_{s}\right)_{t \in \mathbb{R}_{+}}$takes the form $\left(\int_{0}^{t} Y_{s} \mathrm{~d} s\right)_{t \in \mathbb{R}_{+}}$, using (5.1) and Theorem B.1, we have $\widehat{b}_{T} \stackrel{\text { a.s. }}{\longrightarrow} b$ as $T \rightarrow \infty$, as desired.

In the critical case the description of the asymptotic behavior of the MLE in question remains open.

\section{Asymptotic behavior of the MLE in the supercritical case}

7.1 Theorem. Let $a \in \mathbb{R}_{+}, \quad b \in \mathbb{R}_{--}, \quad \sigma \in \mathbb{R}_{+}, \quad \delta \in \mathbb{R}_{++}$, and $\alpha \in(1,2)$. Let $\left(Y_{t}\right)_{t \in \mathbb{R}_{+}}$be the unique strong solution of the $S D E(1.1)$ satisfying $\mathbb{P}\left(Y_{0}=y_{0}\right)=1$ with some $y_{0} \in \mathbb{R}_{+}$.

(i) Then there exists a random variable $V$ with $\mathbb{P}\left(V \in \mathbb{R}_{+}\right)=1$ such that

$$
\mathrm{e}^{b t} Y_{t} \stackrel{\text { a.s. }}{\longrightarrow} V \quad \text { and } \quad \mathrm{e}^{b t} \int_{0}^{t} Y_{u} \mathrm{~d} u \stackrel{\text { a.s. }}{\longrightarrow}-\frac{V}{b} \quad \text { as } t \rightarrow \infty .
$$


(ii) Moreover, the Laplace transform of $V$ takes the form

$$
\mathbb{E}\left(\mathrm{e}^{u V}\right)=\exp \left\{y_{0} \psi_{u}^{*}+\int_{0}^{-\psi_{u}^{*}} \frac{F(z)}{R(z)} \mathrm{d} z\right\}, \quad u \in \mathbb{R}_{-}
$$

where the functions $F$ and $R$ are given in Proposition 2.1, and $\psi_{u}^{*}:=\lim _{t \rightarrow \infty} \psi_{u \mathrm{e}^{b t}, 0}(t)$, where the function $\psi_{u \mathrm{e}^{b t}, 0}: \mathbb{R}_{+} \rightarrow \mathbb{R}_{-}$is given by (3.1).

(iii) Further, $\psi_{0}^{*}=0$ and $\psi_{u}^{*}=-K^{-1}(-u)$ for all $u \in \mathbb{R}_{--}$, where $K^{-1}$ denotes the inverse of the strictly increasing function $K:\left(0, \theta_{0}\right) \rightarrow \mathbb{R}_{++}$given by

$$
K(\lambda):=\lambda \exp \left\{\int_{0}^{\lambda}\left(\frac{b}{R(z)}-\frac{1}{z}\right) \mathrm{d} z\right\}, \quad \lambda \in\left(0, \theta_{0}\right),
$$

where $\theta_{0}=\inf \left\{z \in \mathbb{R}_{++}: R(z) \in \mathbb{R}_{+}\right\} \in \mathbb{R}_{++}$.

(iv) If, in addition, $a \in \mathbb{R}_{++}$, then $\mathbb{P}\left(V \in \mathbb{R}_{++}\right)=1$.

In the next remark we present more properties of $\psi_{u}^{*}, u \in \mathbb{R}_{--}$.

7.2 Remark. (i) For all $\lambda, \theta \in\left(0, \theta_{0}\right)$, we have

$$
\frac{K(\theta)}{K(\lambda)}=\frac{\theta}{\lambda} \exp \left\{\int_{\lambda}^{\theta}\left(\frac{b}{R(z)}-\frac{1}{z}\right) \mathrm{d} z\right\}=\frac{\theta}{\lambda} \exp \left\{\int_{\lambda}^{\theta} \frac{b}{R(z)} \mathrm{d} z-(\log (\theta)-\log (\lambda))\right\}=\exp \left\{\int_{\lambda}^{\theta} \frac{b}{R(z)} \mathrm{d} z\right\},
$$

hence

$$
\int_{\lambda}^{\theta} \frac{b}{R(z)} \mathrm{d} z=\log \left(\frac{K(\theta)}{K(\lambda)}\right) .
$$

Consequently, for all $\lambda \in\left(0, \theta_{0}\right)$ and $u \in \mathbb{R}_{--}$, we conclude

$$
\int_{\lambda}^{-\psi_{u}^{*}} \frac{b}{R(z)} \mathrm{d} z=\log \left(\frac{K\left(-\psi_{u}^{*}\right)}{K(\lambda)}\right)=\log \left(\frac{K\left(K^{-1}(-u)\right)}{K(\lambda)}\right)=\log \left(-\frac{u}{K(\lambda)}\right) .
$$

(ii) Using the formula for the derivative of an inverse function, we have

$$
\frac{\mathrm{d}}{\mathrm{d} u} \psi_{u}^{*}=\frac{1}{K^{\prime}\left(K^{-1}(-u)\right)}=\frac{1}{K^{\prime}\left(-\psi_{u}^{*}\right)}=\frac{R\left(-\psi_{u}^{*}\right)}{b K\left(-\psi_{u}^{*}\right)}, \quad u \in \mathbb{R}_{--} .
$$

Proof of Theorem 7.1. First we check that the function $K$ is well-defined and strictly increasing on $\left(0, \theta_{0}\right)$. Observe that $R(z)=\frac{\sigma^{2}}{2} z^{2}+\frac{\delta^{\alpha}}{\alpha} z^{\alpha}+b z \in \mathbb{R}_{--}$for all $z \in\left(0, \theta_{0}\right)$. Moreover, we have

$$
\frac{b}{R(z)}-\frac{1}{z}=-\frac{\frac{\sigma^{2}}{2} z+\frac{\delta^{\alpha}}{\alpha} z^{\alpha-1}}{R(z)} \in \mathbb{R}_{++}, \quad z \in\left(0, \theta_{0}\right)
$$

Further, $\lim _{z \downarrow 0}\left(\frac{b}{R(z)}-\frac{1}{z}\right) z^{2-\alpha}=-\frac{\delta^{\alpha}}{b \alpha} \in \mathbb{R}_{++}$, thus there exists $z_{1} \in\left(0, \theta_{0}\right)$ such that $\left(\frac{b}{R(z)}-\right.$ $\left.\frac{1}{z}\right) z^{2-\alpha} \leqslant-\frac{2 \delta^{\alpha}}{b \alpha}$ for all $z \in\left(0, z_{1}\right)$. Hence $\int_{0}^{z_{1}}\left(\frac{b}{R(z)}-\frac{1}{z}\right) \mathrm{d} z \leqslant-\frac{2 \delta^{\alpha}}{b \alpha} \int_{0}^{z_{1}} z^{\alpha-2} \mathrm{~d} z<\infty$. The function $\left(0, \theta_{0}\right) \ni z \mapsto \frac{b}{R(z)}-\frac{1}{z} \in \mathbb{R}_{++}$is continuous, thus the integral $\int_{z_{1}}^{\lambda}\left(\frac{b}{R(z)}-\frac{1}{z}\right) \mathrm{d} z$ exists and finite for all $\lambda \in\left(0, \theta_{0}\right)$, and hence the function $K$ is well-defined. Note that the existence and finiteness of the integral $\int_{0}^{\lambda}\left(\frac{b}{R(z)}-\frac{1}{z}\right) \mathrm{d} z$ follows also from Proposition 3.14 and its proof in Li [25], since 
$\int_{1}^{\infty} z \log (z) m(\mathrm{~d} z)<\infty$, where the measure $m$ takes the form $m(\mathrm{~d} z)=\delta^{\alpha} C_{\alpha} z^{-1-\alpha} \mathbb{1}_{\mathbb{R}_{++}}(z) \mathrm{d} z$ Indeed, by partial integration,

$$
\begin{aligned}
& \int_{1}^{\infty} z \log (z) m(\mathrm{~d} z)=\delta^{\alpha} C_{\alpha} \int_{1}^{\infty} \frac{\log (z)}{z^{\alpha}} \mathrm{d} z \\
& =\delta^{\alpha} C_{\alpha}\left(\frac{1}{1-\alpha} \lim _{z \rightarrow \infty} \frac{\log (z)}{z^{\alpha-1}}-\int_{1}^{\infty} \frac{z^{-\alpha}}{1-\alpha} \mathrm{d} z\right)=\frac{\delta^{\alpha} C_{\alpha}}{(1-\alpha)^{2}}<\infty .
\end{aligned}
$$

The function $K$ is strictly increasing on $\left(0, \theta_{0}\right)$, since $\frac{b}{R(z)}-\frac{1}{z} \in \mathbb{R}_{++}$for all $z \in\left(0, \theta_{0}\right)$, and we have $\lim _{\lambda \downarrow 0} K(\lambda)=0$ and $\lim _{\lambda \uparrow \theta_{0}} K(\lambda)=+\infty$, yielding that the range of the function $K$ is $\mathbb{R}_{++}$, hence the inverse $K^{-1}$ is defined on $\mathbb{R}_{++}$. Indeed, $\lim _{z \uparrow \theta_{0}} \frac{R(z)}{z-\theta_{0}}=\lim _{z \uparrow \theta_{0}} \frac{R(z)-R\left(\theta_{0}\right)}{z-\theta_{0}}=R^{\prime}\left(\theta_{0}\right)$. We have $R^{\prime}\left(\theta_{0}\right) \in \mathbb{R}_{++}$, since $R(0)=0$ and $R\left(\theta_{0}\right)=0$ yields the existence of $\theta_{1} \in\left(0, \theta_{0}\right)$ with $R^{\prime}\left(\theta_{1}\right)=0$, and the function $R^{\prime}$ is strictly increasing on $\mathbb{R}_{+}$. Thus there exists $z_{2} \in\left(0, \theta_{0}\right)$ such that $\frac{R(z)}{z-\theta_{0}} \leqslant 2 R^{\prime}\left(\theta_{0}\right)$ for all $z \in\left(z_{2}, \theta_{0}\right)$. Hence, by (17.2), for all $\lambda \in\left(z_{2}, \theta_{0}\right)$, we have

$$
\int_{0}^{\lambda}\left(\frac{b}{R(z)}-\frac{1}{z}\right) \mathrm{d} z \geqslant-\frac{\frac{\sigma^{2}}{2} z_{2}+\frac{\delta^{\alpha}}{\alpha} z_{2}^{\alpha-1}}{2 R^{\prime}\left(\theta_{0}\right)} \int_{z_{2}}^{\lambda} \frac{1}{z-\theta_{0}} \mathrm{~d} z \rightarrow+\infty \quad \text { as } \lambda \uparrow \theta_{0} .
$$

(i) We prove the existence of an appropriate non-negative random variable $V$. We check that

$$
\mathbb{E}\left(Y_{t} \mid \mathcal{F}_{s}^{Y}\right)=\mathbb{E}\left(Y_{t} \mid Y_{s}\right)=\mathrm{e}^{-b(t-s)} Y_{s}+a \mathrm{e}^{b s} \int_{s}^{t} \mathrm{e}^{-b x} \mathrm{~d} x
$$

for all $s, t \in \mathbb{R}_{+}$with $0 \leqslant s \leqslant t$, where $\mathcal{F}_{t}^{Y}:=\sigma\left(Y_{s}, s \in[0, t]\right), \quad t \in \mathbb{R}_{+}$. The first equality follows from the Markov property of the process $\left(Y_{t}\right)_{t \in \mathbb{R}_{+}}$. The second equality is a consequence of the time-homogeneity of the Markov process $Y$ and the fact that

$$
\mathbb{E}\left(Y_{t} \mid Y_{s}=y_{0}\right)=\mathbb{E}\left(Y_{t-s} \mid Y_{0}=y_{0}\right)=\mathrm{e}^{-b(t-s)} y_{0}+a \int_{0}^{t-s} \mathrm{e}^{-b x} \mathrm{~d} x, \quad t \in \mathbb{R}_{+}, \quad y_{0} \in \mathbb{R}_{+},
$$

following from Proposition 2.3. Then

$$
\mathbb{E}\left(\mathrm{e}^{b t} Y_{t} \mid \mathcal{F}_{s}^{Y}\right)=\mathrm{e}^{b s} Y_{s}+a \mathrm{e}^{b(s+t)} \int_{s}^{t} \mathrm{e}^{-b x} \mathrm{~d} x \geqslant \mathrm{e}^{b s} Y_{s}
$$

for all $s, t \in \mathbb{R}_{+}$with $0 \leqslant s \leqslant t$, consequently, the process $\left(\mathrm{e}^{b t} Y_{t}\right)_{t \in \mathbb{R}_{+}}$is a non-negative submartingale with respect to the filtration $\left(\mathcal{F}_{t}^{Y}\right)_{t \in \mathbb{R}_{+}}$. Moreover,

$$
\mathbb{E}\left(\mathrm{e}^{b t} Y_{t}\right)=y_{0}+a \mathrm{e}^{b t} \int_{0}^{t} \mathrm{e}^{-b x} \mathrm{~d} x=y_{0}+a \int_{0}^{t} \mathrm{e}^{b x} \mathrm{~d} x \leqslant y_{0}+a \int_{0}^{\infty} \mathrm{e}^{b x} \mathrm{~d} x=y_{0}-\frac{a}{b}<\infty, \quad t \in \mathbb{R}_{+},
$$

hence, by the submartingale convergence theorem, there exists a non-negative random variable $V$ such that

$$
\mathrm{e}^{b t} Y_{t} \stackrel{\text { a.s. }}{\rightarrow} V \quad \text { as } t \rightarrow \infty .
$$

Further, if $\omega \in \Omega$ such that $\mathrm{e}^{b t} Y_{t}(\omega) \rightarrow V(\omega)$ as $t \rightarrow \infty$, then, by the integral Toeplitz lemma (see Küchler and Sørensen [24, Lemma B.3.2]), we have

$$
\frac{1}{\int_{0}^{t} \mathrm{e}^{-b u} \mathrm{~d} u} \int_{0}^{t} Y_{u}(\omega) \mathrm{d} \omega=\frac{1}{\int_{0}^{t} \mathrm{e}^{-b u} \mathrm{~d} u} \int_{0}^{t} \mathrm{e}^{-b u}\left(\mathrm{e}^{b u} Y_{u}(\omega)\right) \mathrm{d} u \rightarrow V(\omega) \quad \text { as } t \rightarrow \infty .
$$


Here $\int_{0}^{t} \mathrm{e}^{-b u} \mathrm{~d} u=\frac{\mathrm{e}^{-b t}-1}{-b}, t \in \mathbb{R}_{+}$, thus we conclude

$$
\mathrm{e}^{b t} \int_{0}^{t} Y_{u} \mathrm{~d} u=\frac{1-\mathrm{e}^{b t}}{-b} \frac{\int_{0}^{t} Y_{u} \mathrm{~d} u}{\int_{0}^{t} \mathrm{e}^{-b u} \mathrm{~d} u} \stackrel{\text { a.s. }}{\longrightarrow}-\frac{V}{b} \quad \text { as } t \rightarrow \infty .
$$

First proof of (ii), (iii) and (iv). We readily have (7.1) for $u=0$, since then the unique locally bounded solution to the differential equation (3.1) is $\psi_{0,0}(t)=0, \quad t \in \mathbb{R}_{+}$, implying $\psi_{0}^{*}=0$. Convergence $\mathrm{e}^{b t} Y_{t} \stackrel{\text { a.s. }}{\longrightarrow} V$ as $t \rightarrow \infty$ implies $\mathrm{e}^{b t} Y_{t} \stackrel{\mathcal{D}}{\longrightarrow} V$ as $t \rightarrow \infty$, and hence, by the continuity theorem, $\lim _{t \rightarrow \infty} \mathbb{E}\left(\exp \left\{u \mathrm{e}^{b t} Y_{t}\right\}\right)=\mathbb{E}\left(\mathrm{e}^{u V}\right)$ for all $u \in \mathbb{R}_{-}$. By Theorem [3.1, we have

$$
\mathbb{E}\left(\exp \left\{u \mathrm{e}^{b t} Y_{t}\right\}\right)=\exp \left\{y_{0} \psi_{u \mathrm{e}^{b t}, 0}(t)+a \int_{0}^{t} \psi_{u \mathrm{e}^{b t}, 0}(s) \mathrm{d} s\right\}, \quad t \in \mathbb{R}_{+},
$$

thus the limit

$$
\lim _{t \rightarrow \infty} \exp \left\{y_{0} \psi_{u \mathrm{e}^{b t}, 0}(t)+a \int_{0}^{t} \psi_{u \mathrm{e}^{b t}, 0}(s) \mathrm{d} s\right\}=\mathbb{E}\left(\mathrm{e}^{u V}\right) \in(0,1]
$$

exists. Note that the functions $\psi_{u \mathrm{e}^{b t}, 0}, \quad u \in \mathbb{R}_{-}$, do not depend on the values of $a$ and $y_{0}$. Consequently, with $a=0$ and with some $y_{0} \in \mathbb{R}_{++}$, we obtain that the limit $\lim _{t \rightarrow \infty} \exp \left\{\psi_{u e^{b t}, 0}(t)\right\}$ exists, and hence the limit $\lim _{t \rightarrow \infty} \psi_{u e^{b t}, 0}(t)=\psi_{u}^{*}$ exists as well. Using (2.7) and $\psi_{u, 0}(s)=-v_{s}(-u)$ for all $s \in \mathbb{R}_{+}$and $u \in \mathbb{R}_{-}$(see part (i) of Remark [3.2), we obtain

$$
a \int_{0}^{t} \psi_{u \mathrm{e}^{b t}, 0}(s) \mathrm{d} s=\int_{-u \mathrm{e}^{b t}}^{-\psi_{u \mathrm{e}^{b t}, 0}(t)} \frac{F(z)}{R(z)} \mathrm{d} z
$$

for all $t \in \mathbb{R}_{+}$and $u \in \mathbb{R}_{--}$satisfying $-u \mathrm{e}^{b t} \in\left(0, \theta_{0}\right)$, which, together with (7.6), leads to (7.1).

If $t \in \mathbb{R}_{+}$and $u \in \mathbb{R}_{--}$satisfying $-u \mathrm{e}^{b t} \in\left(0, \theta_{0}\right)$, then, by the proof of part (iii) of Proposition 2.1. $-\psi_{u \mathrm{e}^{b t}, 0}(t) \in\left(0, \theta_{0}\right)$, hence, by Proposition 3.3 in Li [25],

$$
\int_{-u \mathrm{e}^{b t}}^{-\psi_{u \mathrm{e}^{b t}, 0}(t)} \frac{1}{R(z)} \mathrm{d} z=-t
$$

It yields that

$$
\int_{-u \mathrm{e}^{b t}}^{-\psi_{u \mathrm{e}^{b t}, 0}(t)}\left(\frac{b}{R(z)}-\frac{1}{z}\right) \mathrm{d} z=-b t-\int_{-u \mathrm{e}^{b t}}^{-\psi_{u \mathrm{e}^{b t}, 0}(t)} \frac{1}{z} \mathrm{~d} z .
$$

The right hand side of (7.7) is

$$
\begin{aligned}
-b t-\int_{-u \mathrm{e}^{b t}}^{-\psi_{u \mathrm{e}^{b t, 0}}(t)} \frac{1}{z} \mathrm{~d} z & =-b t-\left(\log \left(-\psi_{u \mathrm{e}^{b t}, 0}(t)\right)-\log \left(-u \mathrm{e}^{b t}\right)\right) \\
& =\log (-u)-\log \left(-\psi_{u \mathrm{e}^{b t}, 0}(t)\right) .
\end{aligned}
$$

We have already proved that $-\psi_{u \mathrm{e}^{b t}, 0}(s) \in\left(0, \theta_{0}\right)$ for all $s \in \mathbb{R}_{+}$yielding $-\psi_{u}^{*} \in\left[0, \theta_{0}\right]$. Letting $t \rightarrow \infty$ in (7.7), we conclude $-\psi_{u}^{*} \in\left(0, \theta_{0}\right)$. Indeed, $\psi_{u}^{*}=0$ is not possible, since then the left hand side of (17.7) would tend to 0 and the right hand side of (7.7) would tend to $+\infty$ (see (7.8)). Moreover, $\psi_{u}^{*}=-\theta_{0}$ is not possible, since then the left hand side of (17.7) would tend to $\int_{0}^{\theta_{0}}\left(\frac{b}{R(z)}-\frac{1}{z}\right) \mathrm{d} z=+\infty$ (see the beginning of the proof), and the right hand side of (7.7) would tend to $\log (-u)-\log \left(\theta_{0}\right)$ (see (7.8) $)$. Thus $-\psi_{u}^{*} \in\left(0, \theta_{0}\right)$, and letting $t \rightarrow \infty$ in (7.7), we obtain

$$
\int_{0}^{-\psi_{u}^{*}}\left(\frac{b}{R(z)}-\frac{1}{z}\right) \mathrm{d} z=\log (-u)-\log \left(-\psi_{u}^{*}\right)
$$


for all $u \in \mathbb{R}_{--}$. This can be written in the form

$$
u=\psi_{u}^{*} \exp \left\{\int_{0}^{-\psi_{u}^{*}}\left(\frac{b}{R(z)}-\frac{1}{z}\right) \mathrm{d} z\right\} .
$$

Consequently, $u=-K\left(-\psi_{u}^{*}\right)$ for all $u \in \mathbb{R}_{--}$. The function $K$ is strictly increasing on $\left(0, \theta_{0}\right)$, see the beginning of the proof, hence we conclude $\psi_{u}^{*}=-K^{-1}(-u)$ for all $u \in \mathbb{R}_{--}$.

Next, we check that if, in addition, $a \in \mathbb{R}_{++}$, then $\mathbb{P}\left(V \in \mathbb{R}_{++}\right)=1$. The monotone convergence theorem yields $\mathbb{E}\left(\mathrm{e}^{u V}\right) \downarrow \mathbb{E}\left(\mathbb{1}_{\{V=0\}}\right)=\mathbb{P}(V=0)$ as $u \rightarrow-\infty$. We have $\lim _{u \rightarrow-\infty} \psi_{u}^{*}=-\lim _{u \rightarrow-\infty} K^{-1}(-u)=-\theta_{0}$, since $\lim _{\lambda \uparrow \theta_{0}} K(\lambda)=+\infty$, see the beginning of the proof. Consequently, by (7.1), we obtain

$$
\lim _{u \rightarrow-\infty} \mathbb{E}\left(\mathrm{e}^{u V}\right)=\exp \left\{-y_{0} \theta_{0}+\int_{0}^{\theta_{0}} \frac{F(z)}{R(z)} \mathrm{d} z\right\}=0
$$

and hence $\mathbb{P}\left(V \in \mathbb{R}_{++}\right)=1$, since $\int_{0}^{\theta_{0}} \frac{F(z)}{R(z)} \mathrm{d} z=-\infty$. Indeed, as at the beginning of the proof, there exists $z_{2} \in\left(0, \theta_{0}\right)$ such that $\frac{R(z)}{z-\theta_{0}} \leqslant 2 R^{\prime}\left(\theta_{0}\right)$ for all $z \in\left(z_{2}, \theta_{0}\right)$ with $R^{\prime}\left(\theta_{0}\right) \in \mathbb{R}_{++}$, hence $\int_{0}^{\theta_{0}} \frac{F(z)}{R(z)} \mathrm{d} z \leqslant \int_{z_{2}}^{\theta_{0}} \frac{F(z)}{R(z)} \mathrm{d} z \leqslant \frac{a z_{2}}{2 R^{\prime}\left(\theta_{0}\right)} \int_{z_{2}}^{\theta_{0}} \frac{1}{z-\theta_{0}} \mathrm{~d} z=-\infty$.

Second proof of (ii), (iii) and (iv). The idea of this proof is due to Clément Foucart. First, we need to introduce some notations based on $\mathrm{Li}[25]$. For all $t \in \mathbb{R}_{+}$, let $\bar{v}_{t}:=\lim _{\lambda \rightarrow \infty} v_{t}(\lambda)$, which exists in $(0, \infty]$, see Li [25, Theorem 3.5], where $v_{t}(\lambda), t, \lambda \in \mathbb{R}_{+}$, is given in (iii) of Proposition 2.1] Let $\bar{v}:=\lim _{t \rightarrow \infty} \bar{v}_{t}$, which exists in $\mathbb{R}_{+}$, and it is known that $\bar{v}$ is the largest root of the equation $R(z)=0, z \in \mathbb{R}_{+}$, see Li [25, Theorem 3.8]. Indeed, Condition 3.6 in Li [25] holds in our case, since

$$
R(z) \geqslant b z+\frac{\delta^{\alpha}}{\alpha} z^{\alpha} \geqslant \frac{\delta^{\alpha}}{2 \alpha} z^{\alpha}>0 \quad \text { for } \quad z>\left(\frac{-2 b \alpha}{\delta^{\alpha}}\right)^{\frac{1}{\alpha-1}}=: \widetilde{\theta}>0
$$

and

$$
\int_{\widetilde{\theta}}^{\infty} \frac{1}{R(z)} \mathrm{d} z<\frac{2 \alpha}{\delta^{\alpha}} \int_{\widetilde{\theta}}^{\infty} z^{-\alpha} \mathrm{d} z=\frac{2 \alpha \widetilde{\theta}^{1-\alpha}}{\delta^{\alpha}(\alpha-1)}<\infty
$$

Further, by Li [25, page 63], $\bar{v}=\theta_{0}$ and $\theta_{0} \leqslant\left(\frac{-2 b \alpha}{\delta^{\alpha}}\right)^{\frac{1}{\alpha-1}}$. Since $R^{\prime}(0)=b<0, \quad R^{\prime \prime}(z)=$ $\sigma^{2}+\delta^{\alpha}(\alpha-1) z^{\alpha-2} \geqslant 0, \quad z \in \mathbb{R}_{++}$, and $\lim _{z \rightarrow \infty} R(z)=\infty$, we get there is a positive root of $R$ yielding that $\bar{v}=\theta_{0}>0$. For all $t \in \mathbb{R}_{+}$, let $\left[0, \bar{v}_{t}\right) \ni q \mapsto \eta_{t}(q)$ be the inverse function of $\mathbb{R}_{+} \ni \lambda \mapsto v_{t}(\lambda)$, which exists and is strictly monotone increasing, due to the fact that $\mathbb{R}_{+} \ni \lambda \mapsto v_{t}(\lambda)$ is strictly monotone increasing, see Li [25, Proposition 3.1]. By Li [25, Proposition 3.14], we have

$$
\lim _{t \rightarrow \infty} \frac{\eta_{t}(\lambda)}{\mathrm{e}^{b t}}=K(\lambda) \in \mathbb{R}_{++}, \quad \lambda \in\left(0, \theta_{0}\right) .
$$

Indeed, $\quad b \in \mathbb{R}_{--}$, and by (7.3),$\quad \int_{1}^{\infty} z \log (z) m(\mathrm{~d} z)<\infty$, where the measure $m$ takes the form $m(\mathrm{~d} z)=\delta^{\alpha} C_{\alpha} z^{-1-\alpha} \mathbb{1}_{\mathbb{R}_{++}}(z) \mathrm{d} z$. The form of the limit $K(\lambda)$ is

$$
K(\lambda)=\lambda \exp \left\{\int_{0}^{\lambda}\left(\frac{b}{R(z)}-\frac{1}{z}\right) \mathrm{d} z\right\}, \quad \lambda \in\left(0, \theta_{0}\right),
$$

which follows by the proof of Proposition 3.14 in Li [25]. Using (7.4) and (7.9), we have for all $\lambda \in\left(0, \theta_{0}\right)$,

$$
\eta_{t}(\lambda) Y_{t} \stackrel{\text { a.s. }}{\longrightarrow} K(\lambda) V=: U_{\lambda} \quad \text { as } t \rightarrow \infty .
$$


Using the same ideas as in the proofs of Theorems 3.13 and $3.15 \mathrm{in} \mathrm{Li} \mathrm{[25],} \mathrm{we} \mathrm{show} \mathrm{that}$

$$
\mathbb{E}\left(\mathrm{e}^{u U_{\lambda}}\right)=\exp \left\{-y_{0} f(-u, \lambda)+\int_{0}^{f(-u, \lambda)} \frac{F(z)}{R(z)} \mathrm{d} z\right\}, \quad u \in \mathbb{R}_{-}, \quad \lambda \in\left(0, \theta_{0}\right),
$$

where $f(-u, \lambda):=\lim _{t \rightarrow \infty} v_{t}\left(-u \eta_{t}(\lambda)\right) \in\left[0, \theta_{0}\right)$ and, in case of $u \in \mathbb{R}_{--}, f(-u, \lambda) \in\left(0, \theta_{0}\right)$ and it satisfies

$$
\int_{\lambda}^{f(-u, \lambda)} \frac{1}{R(z)} \mathrm{d} z=\frac{\log (-u)}{b} .
$$

The case $u=0$ is trivial, since in this case $f(-u, \lambda)=f(0, \lambda)=\lim _{t \rightarrow \infty} v_{t}(0)=0$, because of $v_{t}(0)=0, t \in \mathbb{R}_{+}$for $u=0$. So we can assume that $u \in \mathbb{R}_{--}$.

Note that the integral $\int_{0}^{f(-u, \lambda)} \frac{F(z)}{R(z)} \mathrm{d} z$ is well-defined. First we check that

$$
\int_{\lambda}^{0} \frac{1}{R(z)} \mathrm{d} z=+\infty, \quad \int_{\lambda}^{\theta_{0}} \frac{1}{R(z)} \mathrm{d} z=-\infty, \quad \lambda \in\left(0, \theta_{0}\right) .
$$

Indeed, we have $\lim _{z \downarrow 0} \frac{R(z)}{z}=b \in \mathbb{R}_{--}$, thus there exists $z_{3} \in(0, \lambda)$ such that $\frac{R(z)}{z} \leqslant \frac{b}{2}$ for all $z \in\left(0, z_{3}\right)$. Hence $\int_{\lambda}^{0} \frac{1}{R(z)} \mathrm{d} z \geqslant \int_{z_{3}}^{0} \frac{2}{b z} \mathrm{~d} z=+\infty$. Moreover, as at the beginning of the proof, there exists $z_{2} \in\left(\lambda, \theta_{0}\right)$ such that $\frac{R(z)}{z-\theta_{0}} \leqslant 2 R^{\prime}\left(\theta_{0}\right)$ for all $z \in\left(z_{2}, \theta_{0}\right)$ with $R^{\prime}\left(\theta_{0}\right) \in \mathbb{R}_{++}$. Hence $\int_{\lambda}^{\theta_{0}} \frac{1}{R(z)} \mathrm{d} z \leqslant \frac{1}{2 R^{\prime}\left(\theta_{0}\right)} \int_{z_{2}}^{\theta_{0}} \frac{1}{z-\theta_{0}} \mathrm{~d} z=-\infty$. Consequently, $f(-u, \lambda)$ can not be a root of the equation $R(z)=0, z \in \mathbb{R}_{+}$, i.e., $f(-u, \lambda) \notin\left\{0, \theta_{0}\right\}$, since otherwise, by (7.13), the left hand side of (7.12) would be $\pm \infty$, but the right hand side of (7.12) is a real number. Hence, using the same argument as in the proof of (2.7), we have $f(-u, \lambda) \in\left(0, \theta_{0}\right)$. The integrand $\frac{F}{R}$ is continuous on $[0, f(-u, \lambda)]$, since in case of $a=0$ the integrand is zero, and in case of $a \in \mathbb{R}_{++}$, by the definition of $\theta_{0}$, we have $R(z)<0$ for all $z \in\left(0, \theta_{0}\right)$ and hence for all $(0, f(-u, \lambda)]$, and $\lim _{z \downarrow 0} \frac{F(z)}{R(z)}=\frac{a}{b} \in \mathbb{R}_{--}$.

Next, by (7.10), we have $\eta_{t}(\lambda) Y_{t} \stackrel{\mathcal{D}}{\longrightarrow} U_{\lambda}$ as $t \rightarrow \infty$ for all $\lambda \in\left(0, \theta_{0}\right)$, and, by continuity theorem and (2.4), for all $u \in \mathbb{R}_{-}$and $\lambda \in\left(0, \theta_{0}\right)$, we get

$$
\mathbb{E}\left(\mathrm{e}^{u U_{\lambda}}\right)=\lim _{t \rightarrow \infty} \mathbb{E}\left(\exp \left\{u \eta_{t}(\lambda) Y_{t}\right\}\right)=\lim _{t \rightarrow \infty} \exp \left\{-y_{0} v_{t}\left(-u \eta_{t}(\lambda)\right)+\int_{-u \eta_{t}(\lambda)}^{v_{t}\left(-u \eta_{t}(\lambda)\right)} \frac{F(z)}{R(z)} \mathrm{d} z\right\},
$$

since $\eta_{t}(\lambda) \downarrow 0$ as $t \rightarrow \infty$ (see Li [25, proof of Proposition 3.14]), and hence for all $u \in \mathbb{R}_{--}$, we have $-u \eta_{t}(\lambda) \in(0, \bar{v})=\left(0, \theta_{0}\right)$ for sufficiently large $t$. Recall that, by formula (3.23) in Li [25], if $\eta_{t}(\lambda)$ and $-u \eta_{t}(\lambda)$ belong to $\left(0, \theta_{0}\right)=(0, \bar{v})$, where $\lambda \in(0, \bar{v})$, then

$$
\int_{\lambda}^{v_{t}\left(-u \eta_{t}(\lambda)\right)} \frac{1}{R(z)} \mathrm{d} z=\int_{\eta_{t}(\lambda)}^{-u \eta_{t}(\lambda)} \frac{1}{R(z)} \mathrm{d} z
$$

Since $\eta_{t}(\lambda) \downarrow 0$ as $t \rightarrow \infty$, for all $u \in \mathbb{R}_{--}$, we have $\eta_{t}(\lambda),-u \eta_{t}(\lambda) \in(0, \bar{v})$ for sufficiently large $t$, and hence for all $u \in \mathbb{R}_{--}$,

$$
\lim _{t \rightarrow \infty} \int_{\lambda}^{v_{t}\left(-u \eta_{t}(\lambda)\right)} \frac{1}{R(z)} \mathrm{d} z=\lim _{t \rightarrow \infty} \int_{\eta_{t}(\lambda)}^{-u \eta_{t}(\lambda)} \frac{1}{R(z)} \mathrm{d} z=\lim _{t \rightarrow \infty} \int_{\eta_{t}(\lambda)}^{-u \eta_{t}(\lambda)} \frac{1}{b z} \mathrm{~d} z=\lim _{t \rightarrow \infty} \frac{\log (-u)}{b}=\frac{\log (-u)}{b},
$$

where we used that $\lim _{z \downarrow 0} \frac{R(z)}{b z}=1$. The function $(0, \bar{v}) \ni x \mapsto \int_{\lambda}^{x} \frac{1}{R(z)} \mathrm{d} z=: G(x)$ is continuous and strictly decreasing, hence its inverse is also continuous and strictly decreasing, implying that for all $u \in \mathbb{R}_{--}$and $\lambda \in(0, \bar{v})$, the limit

$$
f(-u, \lambda)=\lim _{t \rightarrow \infty} v_{t}\left(-u \eta_{t}(\lambda)\right)=\lim _{t \rightarrow \infty} G^{-1}\left(G\left(v_{t}\left(-u \eta_{t}(\lambda)\right)\right)\right)=G^{-1}\left(\frac{\log (-u)}{b}\right)
$$


exists and it satisfies (7.12), where $G^{-1}$ denotes the inverse of $G$, since, by (7.13), the range of $G$ is $\mathbb{R}$. Consequently, using the continuity of the integral upper limit function, the fact that $\eta_{t}(\lambda) \downarrow 0$ as $t \rightarrow \infty$, and (7.14), we have (7.11), as desired. Using that $K(\lambda) \in \mathbb{R}_{++}$and $V=U_{\lambda} / K(\lambda)$, we have $\mathbb{E}\left(\mathrm{e}^{u V}\right)=\mathbb{E}\left(\mathrm{e}^{u U_{\lambda} / K(\lambda)}\right), u \in \mathbb{R}_{-}$, and then (7.11) yields (7.1).

We point out that, in the second proof of (17.1), we were not able to use directly (7.4), and that's why the usage of $\eta_{t}(\lambda)$ in the argument above is really essential for us.

Next, we check that if, in addition, $a \in \mathbb{R}_{++}$, then $\mathbb{P}\left(V \in \mathbb{R}_{++}\right)=1$. By the law of total probability, $\mathbb{E}\left(\mathrm{e}^{u U_{\lambda}}\right)=1 \cdot \mathbb{P}\left(\mathrm{e}^{-U_{\lambda}}=1\right)+\mathbb{E}\left(\mathrm{e}^{u U_{\lambda}} \mid \mathrm{e}^{-U_{\lambda}} \neq 1\right) \mathbb{P}\left(\mathrm{e}^{-U_{\lambda}} \neq 1\right), u \in \mathbb{R}_{-}$, and hence, by the dominated convergence theorem and (17.11),

$$
\begin{aligned}
\mathbb{P}\left(\mathrm{e}^{-U_{\lambda}}=1\right) & =\lim _{u \rightarrow-\infty} \mathbb{E}\left(\mathrm{e}^{u U_{\lambda}}\right)=\lim _{u \rightarrow-\infty} \exp \left\{-y_{0} f(-u, \lambda)+\int_{0}^{f(-u, \lambda)} \frac{F(z)}{R(z)} \mathrm{d} z\right\} \\
& =\exp \left\{-y_{0} \theta_{0}+\int_{0}^{\theta_{0}} \frac{F(z)}{R(z)} \mathrm{d} z\right\}
\end{aligned}
$$

where we used that, by (7.13), $\lim _{u \rightarrow-\infty} f(-u, \lambda)=\lim _{u \rightarrow-\infty} G^{-1}\left(\frac{\log (-u)}{b}\right)=\lim _{y \rightarrow-\infty} G^{-1}(y)=$ $\theta_{0}=\bar{v}$ (see also Li [25, proof of Theorem 3.15]). In case of $a \in \mathbb{R}_{++}$, we have $\int_{0}^{\theta_{0}} \frac{F(z)}{R(z)} \mathrm{d} z=-\infty$ (see the end of the first proof of (iv)), and hence $\mathbb{P}\left(\mathrm{e}^{-U_{\lambda}}=1\right)=0$. This yields that, in case of $a \in \mathbb{R}_{++}, \mathbb{P}\left(U_{\lambda}=0\right)=0$ and, since $K(\lambda) \in \mathbb{R}_{++}$, we have $\mathbb{P}(V=0)=0$, i.e., $\mathbb{P}\left(V \in \mathbb{R}_{++}\right)=1$.

In the next remark we specialize Theorem 7.1 to the case $\sigma=0$.

7.3 Remark. Under the conditions of Theorem 7.1, in case of $\sigma=0$, using part (ii) of Theorem 7.1, we derive the Laplace transform of $V$, which results in an explicit expression. Recall that the function $\psi_{u e^{b t}, 0}: \mathbb{R}_{+} \rightarrow \mathbb{R}_{-}$is the unique locally bounded solution to the differential equation

$$
\psi_{u \mathrm{e}^{b t}, 0}^{\prime}(s)=\frac{\delta^{\alpha}}{\alpha}\left(-\psi_{u \mathrm{e}^{b t}, 0}(s)\right)^{\alpha}-b \psi_{u \mathrm{e}^{b t}, 0}(s), \quad s \in \mathbb{R}_{+}, \quad \psi_{u \mathrm{e}^{b t}, 0}(0)=u \mathrm{e}^{b t} .
$$

We have to determine the limit $\lim _{t \rightarrow \infty} \psi_{u \mathrm{e}^{b t}, 0}(t)$. If $u=0$, then the unique locally bounded solution of the differential equation (7.15) is $\psi_{u \mathrm{e}^{b t}, 0}(s)=0, s \in \mathbb{R}_{+}$, and hence in this case $\lim _{t \rightarrow \infty} \psi_{u \mathrm{e}^{b t}, 0}(t)=0$. In what follows, let us suppose that $u \in \mathbb{R}_{--}$. The unique solution of the differential equation (7.15) (which can be transformed into a Bernoulli differential equation) is

$$
\psi_{u \mathrm{e}^{b t}, 0}(s)=-\left(\left(\left(-u \mathrm{e}^{b t}\right)^{1-\alpha}+\frac{\delta^{\alpha}}{b \alpha}\right) \mathrm{e}^{b(\alpha-1) s}-\frac{\delta^{\alpha}}{b \alpha}\right)^{\frac{1}{1-\alpha}}, \quad s \in \mathbb{R}_{+},
$$

and consequently, by part (ii) of Theorem 7.1 ,

$$
\psi_{u}^{*}=\lim _{t \rightarrow \infty} \psi_{u e^{b t}, 0}(t)=-\left((-u)^{1-\alpha}-\frac{\delta^{\alpha}}{b \alpha}\right)^{\frac{1}{1-\alpha}}, \quad u \in \mathbb{R}_{--},
$$

and hence

$$
\mathbb{E}\left(\mathrm{e}^{u V}\right)=\exp \left\{-y_{0}\left((-u)^{1-\alpha}-\frac{\delta^{\alpha}}{b \alpha}\right)^{\frac{1}{1-\alpha}}+a \int_{0}^{\left((-u)^{1-\alpha}-\frac{\delta^{\alpha}}{b \alpha}\right)^{\frac{1}{1-\alpha}}} \frac{1}{\frac{\delta^{\alpha}}{\alpha} z^{\alpha-1}+b} \mathrm{~d} z\right\}, \quad u \in \mathbb{R}_{--} .
$$


We can derive the above formula for $\psi_{u}^{*}, u \in \mathbb{R}_{--}$, using part (iii) of Theorem 7.1 as well. We have $\theta_{0}=\left(\frac{-b \alpha}{\delta^{\alpha}}\right)^{\frac{1}{\alpha-1}}$ and, by (7.2),

$$
\begin{aligned}
\int_{0}^{\lambda}\left(\frac{b}{R(z)}-\frac{1}{z}\right) \mathrm{d} z & =-\int_{0}^{\lambda} \frac{\frac{\delta^{\alpha}}{\alpha} z^{\alpha-2}}{\frac{\delta^{\alpha}}{\alpha} z^{\alpha-1}+b} \mathrm{~d} z=-\frac{1}{\alpha-1}\left(\log \left(\frac{\delta^{\alpha}}{\alpha} \lambda^{\alpha-1}+b\right)-\log (b)\right) \\
& =\log \left[\left(\frac{\frac{\delta^{\alpha}}{\alpha} \lambda^{\alpha-1}+b}{b}\right)^{-\frac{1}{\alpha-1}}\right]
\end{aligned}
$$

for all $\lambda \in\left(0, \theta_{0}\right)$. Consequently,

$$
K(\lambda)=\lambda\left(\frac{\delta^{\alpha}}{b \alpha} \lambda^{\alpha-1}+1\right)^{-\frac{1}{\alpha-1}}=\left(\frac{\delta^{\alpha}}{b \alpha}+\lambda^{1-\alpha}\right)^{\frac{1}{1-\alpha}}, \quad \lambda \in\left(0, \theta_{0}\right),
$$

thus

$$
\psi_{u}^{*}=-K^{-1}(-u)=-\left((-u)^{1-\alpha}-\frac{\delta^{\alpha}}{b \alpha}\right)^{\frac{1}{1-\alpha}}, \quad u \in \mathbb{R}_{--}
$$

7.4 Theorem. Let $a \in \mathbb{R}_{++}, \quad b \in \mathbb{R}_{--}, \quad \sigma, \delta \in \mathbb{R}_{++}$, and $\alpha \in(1,2)$. Let $\left(Y_{t}\right)_{t \in \mathbb{R}_{+}}$be the unique strong solution of the $S D E(1.1)$ satisfying $\mathbb{P}\left(Y_{0}=y_{0}\right)=1$ with some $y_{0} \in \mathbb{R}_{+}$. Then the MLE of $b$ is strongly consistent and asymptotically mixed normal, i.e., $\widehat{b}_{T} \stackrel{\text { a.s. }}{\longrightarrow} b$ as $T \rightarrow \infty$, and

$$
\mathrm{e}^{-b T / 2}\left(\widehat{b}_{T}-b\right) \stackrel{\mathcal{D}}{\longrightarrow} \sigma Z\left(-\frac{V}{b}\right)^{-1 / 2} \quad \text { as } T \rightarrow \infty,
$$

where $V$ is a positive random variable having Laplace transform given in (7.1), and $Z$ is a standard normally distributed random variable, independent of $V$.

With a random scaling, we have

$$
\frac{1}{\sigma}\left(\int_{0}^{T} Y_{s} \mathrm{~d} s\right)^{1 / 2}\left(\widehat{b}_{T}-b\right) \stackrel{\mathcal{D}}{\longrightarrow} \mathcal{N}(0,1) \quad \text { as } T \rightarrow \infty .
$$

Proof. By Proposition 4.2, there exists a unique MLE $\widehat{b}_{T}$ of $b$ for all $T \in \mathbb{R}_{++}$which takes the form given in (4.3). By Theorem 7.1, $\mathrm{e}^{b t} \int_{0}^{t} Y_{s} \mathrm{~d} s \stackrel{\text { a.s. }}{\longrightarrow}-\frac{V}{b}$ as $t \rightarrow \infty$, where $\mathbb{P}\left(V \in \mathbb{R}_{++}\right)=1$, and hence

$$
\int_{0}^{t} Y_{s} \mathrm{~d} s=\mathrm{e}^{-b t} \mathrm{e}^{b t} \int_{0}^{t} Y_{s} \mathrm{~d} s \stackrel{\text { a.s. }}{\rightarrow} \infty \cdot\left(-\frac{V}{b}\right)=\infty \quad \text { as } t \rightarrow \infty .
$$

Since the quadratic variation process of the square integrable martingale $\left(\int_{0}^{t} \sqrt{Y_{s}} \mathrm{~d} W_{s}\right)_{t \in \mathbb{R}_{+}}$takes the form $\left(\int_{0}^{t} Y_{s} \mathrm{~d} s\right)_{t \in \mathbb{R}_{+}}$, using (5.1) and Theorem B.1, we have $\widehat{b}_{T} \stackrel{\text { a.s. }}{\rightarrow} b$ as $T \rightarrow \infty$. Further, by (5.1),

$$
\mathrm{e}^{-b T / 2}\left(\widehat{b}_{T}-b\right)=-\sigma \frac{\mathrm{e}^{b T / 2} \int_{0}^{T} \sqrt{Y_{s}} \mathrm{~d} W_{s}}{\mathrm{e}^{b T} \int_{0}^{T} Y_{s} \mathrm{~d} s}, \quad T \in \mathbb{R}_{++} .
$$

Again, by Theorem 7.1, $\mathrm{e}^{b T} \int_{0}^{T} Y_{s} \mathrm{~d} s \stackrel{\text { a.s. }}{\longrightarrow}-\frac{V}{b}$ as $T \rightarrow \infty$, and using Theorem B.2 with $\eta:=\left(-\frac{V}{b}\right)^{1 / 2}$ and $v=-\frac{V}{b}$ we have

$$
\left(\mathrm{e}^{b T / 2} \int_{0}^{T} \sqrt{Y_{s}} \mathrm{~d} W_{s},-\frac{V}{b}\right) \stackrel{\mathcal{D}}{\longrightarrow}\left(\left(-\frac{V}{b}\right)^{1 / 2} Z,-\frac{V}{b}\right) \quad \text { as } T \rightarrow \infty .
$$


Consequently, by the continuous mapping theorem, $\mathrm{e}^{-b T / 2}\left(\widehat{b}_{T}-b\right) \stackrel{\mathcal{D}}{\longrightarrow}-\sigma Z\left(\frac{-V}{b}\right)^{-1 / 2}$ as $T \rightarrow \infty$, yielding the first assertion.

Applying again (7.16) and the continuous mapping theorem, we obtain

$$
\begin{aligned}
\frac{1}{\sigma}\left(\int_{0}^{T} Y_{s} \mathrm{~d} s\right)^{1 / 2}\left(\widehat{b}_{T}-b\right) & =-\left(\mathrm{e}^{b T} \int_{0}^{T} Y_{s} \mathrm{~d} s\right)^{-1 / 2} \mathrm{e}^{b T / 2} \int_{0}^{T} \sqrt{Y_{s}} \mathrm{~d} W_{s} \\
& \stackrel{\mathcal{D}}{\longrightarrow}-\left(-\frac{V}{b}\right)^{-1 / 2}\left(-\frac{V}{b}\right)^{1 / 2} Z=-Z \stackrel{\mathcal{D}}{=} \mathcal{N}(0,1) \quad \text { as } T \rightarrow \infty,
\end{aligned}
$$

as desired.

7.5 Remark. Under the conditions of Theorem 7.1, in case of $a \in \mathbb{R}_{++},-\log \left(\frac{Y_{t+1}}{Y_{t}}\right), t \in \mathbb{R}_{++}$, and $-\frac{Y_{t}}{\int_{0}^{t} Y_{s} \mathrm{~d} s}, t \in \mathbb{R}_{++}$, are strongly consistent estimators of $b$ as well. Indeed, by Theorem 7.1, using that $\mathbb{P}\left(V \in \mathbb{R}_{++}\right)=1$ and $\mathbb{P}\left(\int_{0}^{t} Y_{s} \mathrm{~d} s \in \mathbb{R}_{++}\right)=1, t \in \mathbb{R}_{++}$(see (v) of Proposition 2.1), in case of $a \in \mathbb{R}_{++}$,

$$
-\log \left(\frac{Y_{t+1}}{Y_{t}}\right)=-\log \left(\mathrm{e}^{-b} \frac{\mathrm{e}^{b(t+1)} Y_{t+1}}{\mathrm{e}^{b t} Y_{t}}\right) \stackrel{\text { a.s. }}{\longrightarrow}-\log \left(\mathrm{e}^{-b} \frac{V}{V}\right)=b \quad \text { as } t \rightarrow \infty,
$$

and

$$
-\frac{Y_{t}}{\int_{0}^{t} Y_{s} \mathrm{~d} s}=-\frac{\mathrm{e}^{b t} Y_{t}}{\mathrm{e}^{b t} \int_{0}^{t} Y_{s} \mathrm{~d} s} \stackrel{\text { a.s. }}{\longrightarrow}-\frac{V}{-\frac{V}{b}}=b \quad \text { as } t \rightarrow \infty .
$$

\section{Appendices}

\section{A Likelihood-ratio process}

Based on Jacod and Shiryaev [18, see also Jacod and Mémin [16], Sørensen [36] and Luschgy [30], we recall certain sufficient conditions for the absolute continuity of probability measures induced by semimartingales together with a representation of the corresponding Radon-Nikodym derivative (likelihood-ratio process).

Let $D\left(\mathbb{R}_{+}, \mathbb{R}^{d}\right)$ denote the space of $\mathbb{R}^{d}$-valued càdlàg functions defined on $\mathbb{R}_{+}$. Let $\left(\eta_{t}\right)_{t \in \mathbb{R}_{+}}$ denote the canonical process $\eta_{t}(\omega):=\omega(t), \omega \in D\left(\mathbb{R}_{+}, \mathbb{R}^{d}\right), t \in \mathbb{R}_{+}$. Put $\mathcal{F}_{t}^{\eta}:=\sigma\left(\eta_{s}, s \in[0, t]\right)$, $t \in \mathbb{R}_{+}$, and

$$
\mathcal{D}_{t}\left(\mathbb{R}_{+}, \mathbb{R}^{d}\right):=\bigcap_{\varepsilon \in \mathbb{R}_{++}} \mathcal{F}_{t+\varepsilon}^{\eta}, \quad t \in \mathbb{R}_{+}, \quad \mathcal{D}\left(\mathbb{R}_{+}, \mathbb{R}^{d}\right):=\sigma\left(\bigcup_{t \in \mathbb{R}_{+}} \mathcal{F}_{t}^{\eta}\right)
$$

Let $\Psi \subset \mathbb{R}^{k}$ be an arbitrary non-empty set, and let $\mathbb{P}_{\boldsymbol{\psi}}, \boldsymbol{\psi} \in \Psi$, are probability measures on the canonical space $\left(D\left(\mathbb{R}_{+}, \mathbb{R}^{d}\right), \mathcal{D}\left(\mathbb{R}_{+}, \mathbb{R}^{d}\right)\right)$. Suppose that for each $\boldsymbol{\psi} \in \Psi$, under $\mathbb{P}_{\boldsymbol{\psi}}$, the canonical process $\left(\eta_{t}\right)_{t \in \mathbb{R}_{+}}$is a semimartingale with semimartingale characteristics $\left(B^{(\psi)}, C, \nu^{(\psi)}\right)$ associated with a fixed Borel measurable truncation function $h: \mathbb{R}^{d} \rightarrow \mathbb{R}^{d}$, see Jacod and Shiryaev [18, Definition 
II.2.6 and Remark II.2.8]. Namely, $C_{t}:=\left\langle\left(\eta^{\text {cont }}\right)^{(\psi)}\right\rangle_{t}, \quad t \in \mathbb{R}_{+}$, where $\left(\left\langle\left(\eta^{\text {cont }}\right)^{(\psi)}\right\rangle_{t}\right)_{t \in \mathbb{R}_{+}}$denotes the (predictable) quadratic variation process (with values in $\mathbb{R}^{d \times d}$ ) of the continuous martingale part $\left(\eta^{\text {cont }}\right)^{(\psi)}$ of $\eta$ under $\mathbb{P}_{\boldsymbol{\psi}}, \nu^{(\boldsymbol{\psi})}$ is the compensator of the integer-valued random measure $\mu^{\eta}$ on $\mathbb{R}_{+} \times \mathbb{R}^{d}$ associated with the jumps of $\eta$ under $\mathbb{P}_{\psi}$ given by

$$
\mu^{\eta}(\omega, \mathrm{d} t, \mathrm{~d} \boldsymbol{x}):=\sum_{s \in \mathbb{R}_{+}} \mathbb{1}_{\left\{\Delta \eta_{s}(\omega) \neq \mathbf{0}\right\}} \varepsilon_{\left(s, \Delta \eta_{s}(\omega)\right)}(\mathrm{d} t, \mathrm{~d} \boldsymbol{x}), \quad \omega \in D\left(\mathbb{R}_{+}, \mathbb{R}^{d}\right),
$$

where $\varepsilon_{(t, \boldsymbol{x})}$ denotes the Dirac measure at the point $(t, \boldsymbol{x}) \in \mathbb{R}_{+} \times \mathbb{R}^{d}$, and $\Delta \eta_{t}:=\eta_{t}-\eta_{t-}, t \in \mathbb{R}_{++}$, $\Delta \eta_{0}:=\mathbf{0}$, and $B^{(\psi)}$ is the predictable process (with values in $\mathbb{R}^{d}$ having finite variation over each finite interval $\left.[0, t], t \in \mathbb{R}_{+}\right)$appearing in the canonical decomposition

$$
\widetilde{\eta}_{t}=\eta_{0}+M_{t}^{(\psi)}+B_{t}^{(\psi)}, \quad t \in \mathbb{R}_{+},
$$

of the special semimartingale $\left(\widetilde{\eta}_{t}\right)_{t \in \mathbb{R}_{+}}$under $\mathbb{P}_{\boldsymbol{\psi}}$ given by

$$
\widetilde{\eta}_{t}:=\eta_{t}-\sum_{s \in[0, t]}\left(\eta_{s}-h\left(\Delta \eta_{s}\right)\right), \quad t \in \mathbb{R}_{+},
$$

where $\left(M_{t}^{(\psi)}\right)_{t \in \mathbb{R}_{+}}$is a local martingale with $M_{0}^{(\boldsymbol{\psi})}=\mathbf{0}$. We call the attention that, by our assumption, the process $C=\left\langle\left(\eta^{\text {cont }}\right)^{(\psi)}\right\rangle$ does not depend on $\boldsymbol{\psi}$, although $\left(\eta^{\text {cont }}\right)^{(\boldsymbol{\psi})}$ might depend on $\boldsymbol{\psi}$. In addition, assume that $\mathbb{P}_{\boldsymbol{\psi}}\left(\nu^{(\psi)}\left(\{t\} \times \mathbb{R}^{d}\right)=0\right)=1$ for every $\boldsymbol{\psi} \in \Psi, t \in \mathbb{R}_{+}$, and $\mathbb{P}_{\boldsymbol{\psi}}\left(\eta_{0}=\boldsymbol{x}_{0}\right)=1$ with some $\boldsymbol{x}_{0} \in \mathbb{R}^{d}$ for every $\boldsymbol{\psi} \in \Psi$. Note that we have the semimartingale representation

$$
\begin{aligned}
\eta_{t}= & \boldsymbol{x}_{0}+B_{t}^{(\boldsymbol{\psi})}+\left(\eta^{\mathrm{cont}}\right)_{t}^{(\boldsymbol{\psi})}+\int_{0}^{t} \int_{\mathbb{R}^{d}} h(\boldsymbol{x})\left(\mu^{\eta}-\nu^{(\boldsymbol{\psi})}\right)(\mathrm{d} s, \mathrm{~d} \boldsymbol{x}) \\
& +\int_{0}^{t} \int_{\mathbb{R}^{d}}(\boldsymbol{x}-h(\boldsymbol{x})) \mu^{\eta}(\mathrm{d} s, \mathrm{~d} \boldsymbol{x}), \quad t \in \mathbb{R}_{+},
\end{aligned}
$$

of $\eta$ under $\mathbb{P}_{\boldsymbol{\psi}}$, see Jacod and Shiryaev [18, Theorem II.2.34]. Moreover, for each $\boldsymbol{\psi} \in \Psi$, let us choose a nondecreasing, continuous, adapted process $\left(F_{t}^{(\psi)}\right)_{t \in \mathbb{R}_{+}}$with $F_{0}^{(\psi)}=0$ and a predictable process $\left(c_{t}^{(\psi)}\right)_{t \in \mathbb{R}_{+}}$with values in the set of all symmetric positive semidefinite $d \times d$ matrices such that

$$
C_{t}=\int_{0}^{t} c_{s}^{(\psi)} \mathrm{d} F_{s}^{(\psi)}
$$

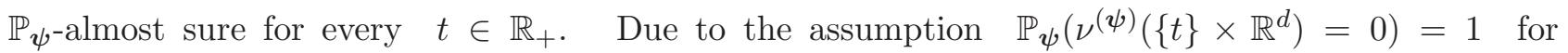
every $\boldsymbol{\psi} \in \Psi, \quad t \in \mathbb{R}_{+}$, such choices of $\left(F_{t}^{(\boldsymbol{\psi})}\right)_{t \in \mathbb{R}_{+}}$and $\left(c_{t}^{(\boldsymbol{\psi})}\right)_{t \in \mathbb{R}_{+}}$are possible, see Jacod and Shiryaev [18, Proposition II.2.9 and Corollary II.1.19]. Let $\mathcal{P}$ denote the predictable $\sigma$-algebra on $D\left(\mathbb{R}_{+}, \mathbb{R}^{d}\right) \times \mathbb{R}_{+}$. Assume also that for every $\boldsymbol{\psi}, \widetilde{\boldsymbol{\psi}} \in \Psi$, there exist a $\mathcal{P} \otimes \mathcal{B}\left(\mathbb{R}^{d}\right)$-measurable function $V^{(\widetilde{\boldsymbol{\psi}}, \boldsymbol{\psi})}: D\left(\mathbb{R}_{+}, \mathbb{R}^{d}\right) \times \mathbb{R}_{+} \times \mathbb{R}^{d} \rightarrow \mathbb{R}_{++}$and a predictable $\mathbb{R}^{d}$-valued process $\beta^{(\widetilde{\boldsymbol{\psi}}, \boldsymbol{\psi})}$ satisfying

$$
\begin{gathered}
\nu^{(\boldsymbol{\psi})}(\mathrm{d} t, \mathrm{~d} \boldsymbol{x})=V^{(\widetilde{\boldsymbol{\psi}}, \boldsymbol{\psi})}(t, \boldsymbol{x}) \nu^{(\widetilde{\boldsymbol{\psi}})}(\mathrm{d} t, \mathrm{~d} \boldsymbol{x}), \\
\int_{0}^{t} \int_{\mathbb{R}^{d}}\left(\sqrt{V^{(\widetilde{\boldsymbol{\psi}}, \boldsymbol{\psi})}(s, \boldsymbol{x})}-1\right)^{2} \nu^{(\widetilde{\boldsymbol{\psi}})}(\mathrm{d} s, \mathrm{~d} \boldsymbol{x})<\infty, \\
B_{t}^{(\psi)}=B_{t}^{(\widetilde{\boldsymbol{\psi}})}+\int_{0}^{t} c_{s}^{(\boldsymbol{\psi})} \beta_{s}^{(\widetilde{\boldsymbol{\psi}}, \boldsymbol{\psi})} \mathrm{d} F_{s}^{(\boldsymbol{\psi})}+\int_{0}^{t} \int_{\mathbb{R}^{d}}\left(V^{(\widetilde{\boldsymbol{\psi}}, \boldsymbol{\psi})}(s, \boldsymbol{x})-1\right) h(\boldsymbol{x}) \nu^{(\widetilde{\boldsymbol{\psi}})}(\mathrm{d} s, \mathrm{~d} \boldsymbol{x}), \\
\int_{0}^{t}\left(\beta_{s}^{(\widetilde{\boldsymbol{\psi}}, \boldsymbol{\psi})}\right)^{\top} c_{s}^{(\psi)} \beta_{s}^{(\widetilde{\boldsymbol{\psi}}, \boldsymbol{\psi})} \mathrm{d} F_{s}^{(\boldsymbol{\psi})}<\infty,
\end{gathered}
$$




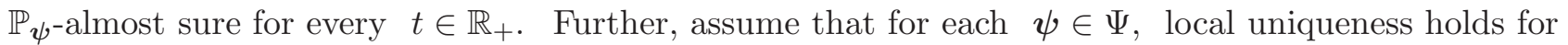
the martingale problem on the canonical space corresponding to the triplet $\left(B^{(\psi)}, C, \nu^{(\psi)}\right)$ with the given initial value $\boldsymbol{x}_{0}$ with $\mathbb{P}_{\boldsymbol{\psi}}$ as its unique solution. Then for each $T \in \mathbb{R}_{+}, \mathbb{P}_{\boldsymbol{\psi}, T}$ is absolutely

continuous with respect to $\mathbb{P}_{\widetilde{\boldsymbol{\psi}}, T}$, where $\mathbb{P}_{\boldsymbol{\psi}, T}:=\left.\mathbb{P}_{\boldsymbol{\psi}}\right|_{\mathcal{D}_{T}\left(\mathbb{R}_{+}, \mathbb{R}^{d}\right)}$ denotes the restriction of $\mathbb{P}_{\boldsymbol{\psi}}$ to $\mathcal{D}_{T}\left(\mathbb{R}_{+}, \mathbb{R}^{d}\right.$ ) (similarly for $\mathbb{P}_{\widetilde{\boldsymbol{\psi}}, T}$ ), and, under $\mathbb{P}_{\widetilde{\boldsymbol{\psi}}, T}$, the corresponding likelihood-ratio process takes the form

$$
\begin{aligned}
\log \frac{\mathrm{d} \mathbb{P}_{\boldsymbol{\psi}, T}}{\mathrm{~d} \mathbb{P}_{\widetilde{\boldsymbol{\psi}}, T}}(\eta)= & \int_{0}^{T}\left(\beta_{s}^{(\widetilde{\boldsymbol{\psi}}, \boldsymbol{\psi})}\right)^{\top} \mathrm{d}\left(\eta^{\operatorname{cont}}\right)_{s}^{(\widetilde{\boldsymbol{\psi}})}-\frac{1}{2} \int_{0}^{T}\left(\beta_{s}^{(\widetilde{\boldsymbol{\psi}}, \boldsymbol{\psi})}\right)^{\top} c_{s}^{(\boldsymbol{\psi})} \beta_{s}^{(\widetilde{\boldsymbol{\psi}}, \boldsymbol{\psi})} \mathrm{d} F_{s}^{(\boldsymbol{\psi})} \\
& +\int_{0}^{T} \int_{\mathbb{R}^{d}}\left(V^{(\widetilde{\boldsymbol{\psi}}, \boldsymbol{\psi})}(s, \boldsymbol{x})-1\right)\left(\mu^{\eta}-\nu^{(\widetilde{\boldsymbol{\psi}})}\right)(\mathrm{d} s, \mathrm{~d} \boldsymbol{x}) \\
& +\int_{0}^{T} \int_{\mathbb{R}^{d}}\left(\log \left(V^{(\widetilde{\boldsymbol{\psi}}, \boldsymbol{\psi})}(s, \boldsymbol{x})\right)-V^{(\widetilde{\boldsymbol{\psi}}, \boldsymbol{\psi})}(s, \boldsymbol{x})+1\right) \mu^{\eta}(\mathrm{d} s, \mathrm{~d} \boldsymbol{x})
\end{aligned}
$$

for all $T \in \mathbb{R}_{++}$, see Jacod and Shiryaev [18, Theorem III.5.34]. A detailed proof of (A.5) using Jacod and Shiryaev [18] can be found in Barczy et al. [2, Appendix A].

\section{B Limit theorems for continuous local martingales}

In what follows we recall some limit theorems for continuous local martingales. We use these limit theorems for studying the asymptotic behavior of the MLE of $b$. First we recall a strong law of large numbers for continuous local martingales.

B.1 Theorem. (Liptser and Shiryaev [28, Lemma 17.4]) Let $\left(\Omega, \mathcal{F},\left(\mathcal{F}_{t}\right)_{t \in \mathbb{R}_{+}}, \mathbb{P}\right)$ be a filtered probability space satisfying the usual conditions. Let $\left(M_{t}\right)_{t \in \mathbb{R}_{+}}$be a square-integrable continuous local martingale with respect to the filtration $\left(\mathcal{F}_{t}\right)_{t \in \mathbb{R}_{+}}$such that $\mathbb{P}\left(M_{0}=0\right)=1$. Let $\left(\xi_{t}\right)_{t \in \mathbb{R}_{+}}$be a progressively measurable process such that

$$
\mathbb{P}\left(\int_{0}^{t} \xi_{u}^{2} \mathrm{~d}\langle M\rangle_{u}<\infty\right)=1, \quad t \in \mathbb{R}_{+},
$$

and

$$
\int_{0}^{t} \xi_{u}^{2} \mathrm{~d}\langle M\rangle_{u} \stackrel{\text { a.s. }}{\rightarrow} \infty \quad \text { as } t \rightarrow \infty
$$

where $\left(\langle M\rangle_{t}\right)_{t \in \mathbb{R}_{+}}$denotes the quadratic variation process of $M$. Then

$$
\frac{\int_{0}^{t} \xi_{u} \mathrm{~d} M_{u}}{\int_{0}^{t} \xi_{u}^{2} \mathrm{~d}\langle M\rangle_{u}} \stackrel{\text { a.s. }}{\longrightarrow} 0 \quad \text { as } t \rightarrow \infty .
$$

If $\left(M_{t}\right)_{t \in \mathbb{R}_{+}}$is a standard Wiener process, the progressive measurability of $\left(\xi_{t}\right)_{t \in \mathbb{R}_{+}}$can be relaxed to measurability and adaptedness to the filtration $\left(\mathcal{F}_{t}\right)_{t \in \mathbb{R}_{+}}$.

The next theorem is about the asymptotic behavior of continuous multivariate local martingales, see van Zanten [37, Theorem 4.1]. 
B.2 Theorem. (van Zanten [37, Theorem 4.1]) Let $\left(\Omega, \mathcal{F},\left(\mathcal{F}_{t}\right)_{t \in \mathbb{R}_{+}}, \mathbb{P}\right)$ be a filtered probability space satisfying the usual conditions. Let $\left(\boldsymbol{M}_{t}\right)_{t \in \mathbb{R}_{+}}$be a d-dimensional square-integrable continuous local martingale with respect to the filtration $\left(\mathcal{F}_{t}\right)_{t \in \mathbb{R}_{+}}$such that $\mathbb{P}\left(\boldsymbol{M}_{0}=\mathbf{0}\right)=1$. Suppose that there exists a function $\boldsymbol{Q}:\left[t_{0}, \infty\right) \rightarrow \mathbb{R}^{d \times d}$ with some $t_{0} \in \mathbb{R}_{+}$such that $\boldsymbol{Q}(t)$ is an invertible (non-random) matrix for all $t \in\left[t_{0}, \infty\right), \lim _{t \rightarrow \infty}\|\boldsymbol{Q}(t)\|=0$ and

$$
\boldsymbol{Q}(t)\langle\boldsymbol{M}\rangle_{t} \boldsymbol{Q}(t)^{\top} \stackrel{\mathbb{P}}{\longrightarrow} \boldsymbol{\eta} \boldsymbol{\eta}^{\top} \quad \text { as } t \rightarrow \infty,
$$

where $\boldsymbol{\eta}$ is a $d \times d$ random matrix. Then, for each $\mathbb{R}^{k}$-valued random vector $\boldsymbol{v}$ defined on $(\Omega, \mathcal{F}, \mathbb{P})$, we have

$$
\left(\boldsymbol{Q}(t) \boldsymbol{M}_{t}, \boldsymbol{v}\right) \stackrel{\mathcal{D}}{\longrightarrow}(\boldsymbol{\eta} \boldsymbol{Z}, \boldsymbol{v}) \quad \text { as } t \rightarrow \infty,
$$

where $\boldsymbol{Z}$ is a d-dimensional standard normally distributed random vector independent of $(\boldsymbol{\eta}, \boldsymbol{v})$.

\section{Some explicit formulae in case of $\alpha=\frac{3}{2}$}

First, in the special case of $\alpha=\frac{3}{2}$, we make explicit the Laplace transform of the stationary distribution in the subcritical and critical cases given in Theorem 2.5 by evaluating the integral in its expression.

C.1 Example. We calculate the Laplace transform of the unique stationary distribution $\pi$ given in Theorem 2.5 in case of $\alpha=\frac{3}{2}$. Let $u \in \mathbb{R}_{--}$. By Theorem 2.5.

$$
\int_{0}^{\infty} \mathrm{e}^{u y} \pi(\mathrm{d} y)=\exp \left\{-a \int_{0}^{-u} \frac{1}{\frac{\sigma^{2}}{2} x+\frac{2 \delta^{\frac{3}{2}}}{3} x^{\frac{1}{2}}+b} \mathrm{~d} x\right\}
$$

By substitution $x=y^{2}$,

$$
\int_{0}^{-u} \frac{1}{\frac{\sigma^{2}}{2} x+\frac{2 \delta^{\frac{3}{2}}}{3} x^{\frac{1}{2}}+b} \mathrm{~d} x=\int_{0}^{(-u)^{\frac{1}{2}}} \frac{2 y}{\frac{\sigma^{2}}{2} y^{2}+\frac{2 \delta^{\frac{3}{2}}}{3} y+b} \mathrm{~d} y .
$$

First we consider the case of $b \in \mathbb{R}_{++}$and $\sigma \in \mathbb{R}_{++}$. Then we can write

$$
\frac{2 y}{\frac{\sigma^{2}}{2} y^{2}+\frac{2 \delta^{\frac{3}{2}}}{3} y+b}=\frac{2}{\sigma^{2}} \cdot \frac{2 y+\frac{4 \delta^{\frac{3}{2}}}{3 \sigma^{2}}}{y^{2}+\frac{4 \delta^{\frac{3}{2}}}{3 \sigma^{2}} y+\frac{2 b}{\sigma^{2}}}-\frac{8 \delta^{\frac{3}{2}}}{3 \sigma^{4}} \cdot \frac{1}{y^{2}+\frac{4 \delta^{\frac{3}{2}}}{3 \sigma^{2}} y+\frac{2 b}{\sigma^{2}}}
$$

We have

$$
\int_{0}^{(-u)^{\frac{1}{2}}} \frac{2 y+\frac{4 \delta^{\frac{3}{2}}}{3 \sigma^{2}}}{y^{2}+\frac{4 \delta^{\frac{3}{2}}}{3 \sigma^{2}} y+\frac{2 b}{\sigma^{2}}} \mathrm{~d} y=\left[\log \left(y^{2}+\frac{4 \delta^{\frac{3}{2}}}{3 \sigma^{2}} y+\frac{2 b}{\sigma^{2}}\right)\right]_{y=0}^{y=(-u)^{\frac{1}{2}}}=\log \left(\frac{\sigma^{2}}{2 b}(-u)+\frac{2 \delta^{\frac{3}{2}}}{3 b}(-u)^{\frac{1}{2}}+1\right) .
$$

Moreover, using

$$
y^{2}+\frac{4 \delta^{\frac{3}{2}}}{3 \sigma^{2}} y+\frac{2 b}{\sigma^{2}}=\left(y+\frac{2 \delta^{\frac{3}{2}}}{3 \sigma^{2}}\right)^{2}+\frac{2 b}{\sigma^{2}}-\frac{4 \delta^{3}}{9 \sigma^{4}},
$$


we obtain

$$
\int \frac{1}{y^{2}+\frac{4 \delta^{\frac{3}{2}}}{3 \sigma^{2}} y+\frac{2 b}{\sigma^{2}}} \mathrm{~d} y= \begin{cases}\frac{1}{\sqrt{\frac{2 b}{\sigma^{2}}-\frac{4 \delta^{3}}{9 \sigma^{4}}}} \arctan \left(\frac{y+\frac{2 \delta^{\frac{3}{2}}}{3 \sigma^{2}}}{\sqrt{\frac{2 b}{\sigma^{2}}-\frac{4 \delta^{3}}{9 \sigma^{4}}}}\right)+C & \text { if } b \in\left(\frac{2 \delta^{3}}{9 \sigma^{2}}, \infty\right), \\ -\frac{1}{y+\frac{2 \delta^{\frac{3}{2}}}{3 \sigma^{2}}}+C & \text { if } b=\frac{2 \delta^{3}}{9 \sigma^{2}}, \\ \frac{1}{\sqrt{\frac{4 \delta^{3}}{9 \sigma^{4}}-\frac{2 b}{\sigma^{2}}}} \log \left(\mid \frac{y+\frac{2 \delta^{\frac{3}{2}}}{3 \sigma^{2}}-\sqrt{\frac{4 \delta^{3}}{9 \sigma^{4}}-\frac{2 b}{\sigma^{2}}}}{y+\frac{2 \delta^{\frac{3}{2}}}{3 \sigma^{2}}+\sqrt{\frac{4 \delta^{3}}{9 \sigma^{4}}-\frac{2 b}{\sigma^{2}}} \mid}\right)+C & \text { if } b \in\left(0, \frac{2 \delta^{3}}{9 \sigma^{2}}\right),\end{cases}
$$

where $C \in \mathbb{R}$. If $b \in\left(\frac{2 \delta^{3}}{9 \sigma^{2}}, \infty\right)$ and $\sigma \in \mathbb{R}_{++}$then, applying the formula $\arctan (u)-\arctan (v)=$ $\arctan \left(\frac{u-v}{1+u v}\right), u, v \in \mathbb{R}_{+}$, we get

$$
\begin{aligned}
\int_{0}^{(-u)^{\frac{1}{2}}} \frac{1}{y^{2}+\frac{4 \delta^{\frac{3}{2}}}{3 \sigma^{2}} y+\frac{2 b}{\sigma^{2}}} \mathrm{~d} y & =\frac{1}{\sqrt{\frac{2 b}{\sigma^{2}}-\frac{4 \delta^{3}}{9 \sigma^{4}}}}\left(\arctan \left(\frac{(-u)^{\frac{1}{2}}+\frac{2 \delta^{\frac{3}{2}}}{3 \sigma^{2}}}{\sqrt{\frac{2 b}{\sigma^{2}}-\frac{4 \delta^{3}}{9 \sigma^{4}}}}\right)-\arctan \left(\frac{\frac{2 \delta^{\frac{3}{2}}}{3 \sigma^{2}}}{\sqrt{\frac{2 b}{\sigma^{2}}-\frac{4 \delta^{3}}{9 \sigma^{4}}}}\right)\right) \\
& =\frac{1}{\sqrt{\frac{2 b}{\sigma^{2}}-\frac{4 \delta^{3}}{9 \sigma^{4}}}} \arctan \left(\frac{\sqrt{\frac{2 b}{\sigma^{2}}-\frac{4 \delta^{3}}{9 \sigma^{4}}}}{\frac{2 b}{\sigma^{2}}(-u)^{-\frac{1}{2}}+\frac{2 \delta^{\frac{3}{2}}}{3 \sigma^{2}}}\right),
\end{aligned}
$$

and hence

$$
\int_{0}^{\infty} \mathrm{e}^{u y} \pi(\mathrm{d} y)=\left(\frac{\sigma^{2}}{2 b}(-u)+\frac{2 \delta^{\frac{3}{2}}}{3 b}(-u)^{\frac{1}{2}}+1\right)^{-\frac{2 a}{\sigma^{2}}} \exp \left\{\frac{\frac{8 a \delta^{\frac{3}{2}}}{3 \sigma^{4}}}{\sqrt{\frac{2 b}{\sigma^{2}}-\frac{4 \delta^{3}}{9 \sigma^{4}}}} \arctan \left(\frac{\sqrt{\frac{2 b}{\sigma^{2}}-\frac{4 \delta^{3}}{9 \sigma^{4}}}}{\frac{2 b}{\sigma^{2}}(-u)^{-\frac{1}{2}}+\frac{2 \delta^{\frac{3}{2}}}{3 \sigma^{2}}}\right)\right\} .
$$

If $b=\frac{2 \delta^{3}}{9 \sigma^{2}}$ and $\sigma \in \mathbb{R}_{++}$, then

$$
\int_{0}^{(-u)^{\frac{1}{2}}} \frac{1}{y^{2}+\frac{4 \delta^{\frac{3}{2}}}{3 \sigma^{2}} y+\frac{2 b}{\sigma^{2}}} \mathrm{~d} y=-\frac{1}{(-u)^{\frac{1}{2}}+\frac{2 \delta^{\frac{3}{2}}}{3 \sigma^{2}}}+\frac{1}{\frac{2 \delta^{\frac{3}{2}}}{3 \sigma^{2}}}=\frac{(-u)^{\frac{1}{2}}}{(-u)^{\frac{1}{2}}+\frac{2 \delta^{\frac{3}{2}}}{3 \sigma^{2}}}=\frac{1}{1+\frac{2 \delta^{\frac{3}{2}}}{3 \sigma^{2}}(-u)^{-\frac{1}{2}}},
$$

and hence

$$
\int_{0}^{\infty} \mathrm{e}^{u y} \pi(\mathrm{d} y)=\left(\frac{9 \sigma^{4}}{4 \delta^{3}}(-u)+\frac{3 \sigma^{2}}{\delta^{\frac{3}{2}}}(-u)^{\frac{1}{2}}+1\right)^{-\frac{2 a}{\sigma^{2}}} \exp \left\{\frac{\frac{8 a \delta^{\frac{3}{2}}}{3 \sigma^{4}}}{1+\frac{2 \delta^{\frac{3}{2}}}{3 \sigma^{2}}(-u)^{-\frac{1}{2}}}\right\} .
$$

If $b \in\left(0, \frac{2 \delta^{3}}{9 \sigma^{2}}\right)$ and $\sigma \in \mathbb{R}_{++}$, then

$$
\begin{aligned}
& \int_{0}^{(-u)^{\frac{1}{2}}} \frac{1}{y^{2}+\frac{4 \delta^{\frac{3}{2}}}{3 \sigma^{2}} y+\frac{2 b}{\sigma^{2}}} \mathrm{~d} y \\
& =\frac{1}{\sqrt{\frac{4 \delta^{3}}{9 \sigma^{4}}-\frac{2 b}{\sigma^{2}}}}\left(\log \left(\frac{(-u)^{\frac{1}{2}}+\frac{2 \delta^{\frac{3}{2}}}{3 \sigma^{2}}-\sqrt{\frac{4 \delta^{3}}{9 \sigma^{4}}-\frac{2 b}{\sigma^{2}}}}{(-u)^{\frac{1}{2}}+\frac{2 \delta^{\frac{3}{2}}}{3 \sigma^{2}}+\sqrt{\frac{4 \delta^{3}}{9 \sigma^{4}}-\frac{2 b}{\sigma^{2}}}}\right)-\log \left(\frac{\frac{2 \delta^{\frac{3}{2}}}{3 \sigma^{2}}-\sqrt{\frac{4 \delta^{3}}{9 \sigma^{4}}-\frac{2 b}{\sigma^{2}}}}{\frac{2 \delta^{\frac{3}{2}}}{3 \sigma^{2}}+\sqrt{\frac{4 \delta^{3}}{9 \sigma^{4}}-\frac{2 b}{\sigma^{2}}}}\right)\right) \\
& =\frac{1}{\sqrt{\frac{4 \delta^{3}}{9 \sigma^{4}}-\frac{2 b}{\sigma^{2}}}} \log \left(\frac{(-u)^{\frac{1}{2}}\left(\frac{2 \delta^{\frac{3}{2}}}{3 \sigma^{2}}+\sqrt{\frac{4 \delta^{3}}{9 \sigma^{4}}-\frac{2 b}{\sigma^{2}}}\right)+\frac{2 b}{\sigma^{2}}}{(-u)^{\frac{1}{2}}\left(\frac{2 \delta^{\frac{3}{2}}}{3 \sigma^{2}}-\sqrt{\frac{4 \delta^{3}}{9 \sigma^{4}}-\frac{2 b}{\sigma^{2}}}\right)+\frac{2 b}{\sigma^{2}}}\right)
\end{aligned}
$$


and hence

$$
\int_{0}^{\infty} \mathrm{e}^{u y} \pi(\mathrm{d} y)=\left(\frac{\sigma^{2}}{2 b}(-u)+\frac{2 \delta^{\frac{3}{2}}}{3 b}(-u)^{\frac{1}{2}}+1\right)^{-\frac{2 a}{\sigma^{2}}}\left(\frac{(-u)^{\frac{1}{2}}\left(\frac{2 \delta^{\frac{3}{2}}}{3 \sigma^{2}}+\sqrt{\frac{4 \delta^{3}}{9 \sigma^{4}}-\frac{2 b}{\sigma^{2}}}\right)+\frac{2 b}{\sigma^{2}}}{(-u)^{\frac{1}{2}}\left(\frac{2 \delta^{\frac{3}{2}}}{3 \sigma^{2}}-\sqrt{\frac{4 \delta^{3}}{9 \sigma^{4}}-\frac{2 b}{\sigma^{2}}}\right)+\frac{2 b}{\sigma^{2}}}\right)^{\frac{\frac{8 a \delta^{\frac{3}{2}}}{3 \sigma^{4}}}{\sqrt{\frac{4 \delta^{3}}{9}-\frac{2 b}{\sigma^{2}}}}} .
$$

Next we consider the case of $b \in \mathbb{R}_{++}$and $\sigma=0$. Then we can write

$$
\frac{2 y}{\frac{2 \delta^{\frac{3}{2}}}{3} y+b}=\frac{3}{\delta^{\frac{3}{2}}}-\frac{\frac{9 b}{2 \delta^{3}}}{y+\frac{3 b}{2 \delta^{\frac{3}{2}}}}
$$

thus

$$
\begin{aligned}
\int_{0}^{(-u)^{\frac{1}{2}}} \frac{2 y}{\frac{2 \delta^{\frac{3}{2}}}{3} y+b} \mathrm{~d} y & =\frac{3}{\delta^{\frac{3}{2}}}(-u)^{\frac{1}{2}}-\frac{9 b}{2 \delta^{3}}\left(\log \left((-u)^{\frac{1}{2}}+\frac{3 b}{2 \delta^{\frac{3}{2}}}\right)-\log \left(\frac{3 b}{2 \delta^{\frac{3}{2}}}\right)\right) \\
& =\frac{3}{\delta^{\frac{3}{2}}}(-u)^{\frac{1}{2}}-\frac{9 b}{2 \delta^{3}} \log \left(\frac{2 \delta^{\frac{3}{2}}}{3 b}(-u)^{\frac{1}{2}}+1\right),
\end{aligned}
$$

and hence

$$
\int_{0}^{\infty} \mathrm{e}^{u y} \pi(\mathrm{d} y)=\exp \left\{-\frac{3 a}{\delta^{\frac{3}{2}}}(-u)^{\frac{1}{2}}\right\}\left(1+\frac{2 \delta^{\frac{3}{2}}}{3 b}(-u)^{\frac{1}{2}}\right)^{\frac{9 b a}{2 \delta^{3}}} .
$$

Next we consider the case of $b=0$ and $\sigma \in \mathbb{R}_{++}$. Let $u \in \mathbb{R}_{-}$. By Theorem 2.5,

$$
\int_{0}^{\infty} \mathrm{e}^{u y} \pi(\mathrm{d} y)=\exp \left\{-a \int_{0}^{-u} \frac{1}{\frac{\sigma^{2}}{2} x+\frac{2 \delta^{\frac{3}{2}}}{3} x^{\frac{1}{2}}} \mathrm{~d} x\right\} .
$$

By substitution $x=y^{2}$,

$$
\int_{0}^{-u} \frac{1}{\frac{\sigma^{2}}{2} x+\frac{2 \delta^{\frac{3}{2}}}{3} x^{\frac{1}{2}}} \mathrm{~d} x=\int_{0}^{(-u)^{\frac{1}{2}}} \frac{2 y}{\frac{\sigma^{2}}{2} y^{2}+\frac{2 \delta^{\frac{3}{2}}}{3} y} \mathrm{~d} y=\int_{0}^{(-u)^{\frac{1}{2}}} \frac{2}{\frac{\sigma^{2}}{2} y+\frac{2 \delta^{\frac{3}{2}}}{3}} \mathrm{~d} y .
$$

Consequently,

$$
\begin{aligned}
\int_{0}^{(-u)^{\frac{1}{2}}} \frac{2}{\frac{\sigma^{2}}{2} y+\frac{2 \delta^{\frac{3}{2}}}{3}} \mathrm{~d} y & =\left[\frac{4}{\sigma^{2}} \log \left(y+\frac{4 \delta^{\frac{3}{2}}}{3 \sigma^{2}}\right)\right]_{y=0}^{y=(-u)^{\frac{1}{2}}}=\frac{4}{\sigma^{2}} \log \left((-u)^{\frac{1}{2}}+\frac{4 \delta^{\frac{3}{2}}}{3 \sigma^{2}}\right)-\frac{4}{\sigma^{2}} \log \left(\frac{4 \delta^{\frac{3}{2}}}{3 \sigma^{2}}\right) \\
& =\frac{4}{\sigma^{2}} \log \left(\frac{3 \sigma^{2}}{4 \delta^{\frac{3}{2}}}(-u)^{\frac{1}{2}}+1\right),
\end{aligned}
$$

hence

$$
\int_{0}^{\infty} \mathrm{e}^{u y} \pi(\mathrm{d} y)=\left(\frac{3 \sigma^{2}}{4 \delta^{\frac{3}{2}}}(-u)^{\frac{1}{2}}+1\right)^{-\frac{4 a}{\sigma^{2}}} .
$$

Finally, by the proof of Theorem 2.5, if $b=0, \quad \sigma=0$ and $\alpha=\frac{3}{2}$, then $\int_{0}^{\infty} \mathrm{e}^{u y} \pi(\mathrm{d} y)=$ $\exp \left\{-\frac{3 a}{\delta^{3 / 2}}(-u)^{1 / 2}\right\}, u \in \mathbb{R}_{-}$. 
C.2 Example. Now we formulate a special case of Theorem 3.1 giving the Laplace transform of $Y_{t}$ in case of $\alpha=\frac{3}{2}$. Let $\left(Y_{t}\right)_{t \in \mathbb{R}_{+}}$be the unique strong solution of the $\operatorname{SDE}(1.1)$ satisfying $\mathbb{P}\left(Y_{0}=y_{0}\right)=1$ with some $y_{0} \in \mathbb{R}_{+}$, with $a \in \mathbb{R}_{+}, \quad b \in \mathbb{R}, \sigma \in \mathbb{R}_{++}, \delta \in \mathbb{R}_{++}$and $\alpha=\frac{3}{2}$. Then, by Theorem 3.1, for all $u \in \mathbb{R}_{-}$,

$$
\mathbb{E}\left(\mathrm{e}^{u Y_{t}}\right)=\exp \left\{\psi_{u, 0}(t) y_{0}+a \int_{0}^{t} \psi_{u, 0}(s) \mathrm{d} s\right\}, \quad t \in \mathbb{R}_{+},
$$

where the function $\psi_{u, 0}: \mathbb{R}_{+} \rightarrow \mathbb{R}_{-}$is the unique locally bounded solution to the differential equation

$$
\psi_{u, 0}^{\prime}(t)=\frac{\sigma^{2}}{2} \psi_{u, 0}(t)^{2}+\frac{2 \delta^{\frac{3}{2}}}{3}\left(-\psi_{u, 0}(t)\right)^{\frac{3}{2}}-b \psi_{u, 0}(t), \quad t \in \mathbb{R}_{+}, \quad \psi_{u, 0}(0)=u
$$

In case of $u=0$, the unique locally bounded solution of (C.2) is $\psi_{0,0}(t)=0, t \in \mathbb{R}_{+}$. Let us consider the function $g_{u}(t):=\left(-\psi_{u, 0}(t)\right)^{\frac{1}{2}} \in \mathbb{R}_{++}, \quad t \in \mathbb{R}_{+}$. Then we have $\psi_{u, 0}(t)=-g_{u}(t)^{2}$, $\psi_{u, 0}(t)^{2}=g_{u}(t)^{4}, \quad\left(-\psi_{u, 0}(t)\right)^{\frac{3}{2}}=g_{u}(t)^{3}$ and $\psi_{u, 0}^{\prime}(t)=-2 g_{u}(t) g_{u}^{\prime}(t)$ for all $t \in \mathbb{R}_{+}$and $u \in \mathbb{R}_{--}$, hence (C.2) yields

$$
g_{u}^{\prime}(t)=-\frac{\sigma^{2}}{4} g_{u}(t)^{3}-\frac{\delta^{\frac{3}{2}}}{3} g_{u}(t)^{2}-\frac{b}{2} g_{u}(t), \quad t \in \mathbb{R}_{+}, \quad g_{u}(0)=(-u)^{\frac{1}{2}} .
$$

In case of $b \in \mathbb{R}_{+}$, (C.3) has a constant solution if and only if $u=0$, and then $g_{0}(t)=\psi_{0,0}(t)=0$ for all $t \in \mathbb{R}_{+}$. In case of $b \in \mathbb{R}_{--}$, (C.3) has a constant solution if and only if $u=0$ or

$$
u=u_{0}:=-\left(-\frac{2 \delta^{\frac{3}{2}}}{3 \sigma^{2}}+\sqrt{\frac{4 \delta^{3}}{9 \sigma^{4}}-\frac{2 b}{\sigma^{2}}}\right)^{2},
$$

and then $g_{0}(t)=\psi_{0,0}(t)=0$ for all $t \in \mathbb{R}_{+}$or $g_{u_{0}}(t)=\left(-u_{0}\right)^{\frac{1}{2}}$, and hence $\psi_{u_{0}, 0}(t)=u_{0}$ for all $t \in \mathbb{R}_{+}$. In the sequel, we suppose that $u \in \mathbb{R}_{--}$, and in case of $b \in \mathbb{R}_{--}$, in addition, we suppose that $u \neq u_{0}$. Then, by separation of variables, we have

$$
\frac{1}{\left(g_{u}^{2}+\frac{4 \delta^{\frac{3}{2}}}{3 \sigma^{2}} g_{u}+\frac{2 b}{\sigma^{2}}\right) g_{u}} \mathrm{~d} g_{u}=-\frac{\sigma^{2}}{4} \mathrm{~d} t .
$$

If $b \neq 0$, then

$$
\begin{aligned}
\frac{1}{\left(g_{u}^{2}+\frac{4 \delta^{\frac{3}{2}}}{3 \sigma^{2}} g_{u}+\frac{2 b}{\sigma^{2}}\right) g_{u}} & =\frac{\sigma^{2}}{2 b g_{u}}-\frac{\frac{\sigma^{2}}{2 b} g_{u}+\frac{2 \delta^{\frac{3}{2}}}{3 b}}{g_{u}^{2}+\frac{4 \delta^{\frac{3}{2}}}{3 \sigma^{2}} g_{u}+\frac{2 b}{\sigma^{2}}} \\
& =\frac{\sigma^{2}}{2 b g_{u}}-\frac{\frac{\sigma^{2}}{2 b} g_{u}+\frac{\delta^{\frac{3}{2}}}{3 b}}{g_{u}^{2}+\frac{4 \delta^{\frac{3}{2}}}{3 \sigma^{2}} g_{u}+\frac{2 b}{\sigma^{2}}}-\frac{\frac{\delta^{\frac{3}{2}}}{3 b}}{g_{u}^{2}+\frac{4 \delta^{\frac{3}{2}}}{3 \sigma^{2}} g_{u}+\frac{2 b}{\sigma^{2}}},
\end{aligned}
$$

and we have

$$
\begin{aligned}
\int\left(\frac{\sigma^{2}}{2 b g_{u}}-\frac{\frac{\sigma^{2}}{2 b} g_{u}+\frac{\delta^{\frac{3}{2}}}{3 b}}{g_{u}^{2}+\frac{4 \delta^{\frac{3}{2}}}{3 \sigma^{2}} g_{u}+\frac{2 b}{\sigma^{2}}}\right) \mathrm{d} g_{u} & =\frac{\sigma^{2}}{2 b} \log \left(\left|g_{u}\right|\right)-\frac{\sigma^{2}}{4 b} \log \left(\left|g_{u}^{2}+\frac{4 \delta^{\frac{3}{2}}}{3 \sigma^{2}} g_{u}+\frac{2 b}{\sigma^{2}}\right|\right)+C \\
& =-\frac{\sigma^{2}}{4 b} \log \left(\left|1+\frac{4 \delta^{\frac{3}{2}}}{3 \sigma^{2} g_{u}}+\frac{2 b}{\sigma^{2} g_{u}^{2}}\right|\right)+C,
\end{aligned}
$$


where $C \in \mathbb{R}$. Moreover, using

$$
g_{u}^{2}+\frac{4 \delta^{\frac{3}{2}}}{3 \sigma^{2}} g_{u}+\frac{2 b}{\sigma^{2}}=\left(g_{u}+\frac{2 \delta^{\frac{3}{2}}}{3 \sigma^{2}}\right)^{2}+\frac{2 b}{\sigma^{2}}-\frac{4 \delta^{3}}{9 \sigma^{4}},
$$

we obtain

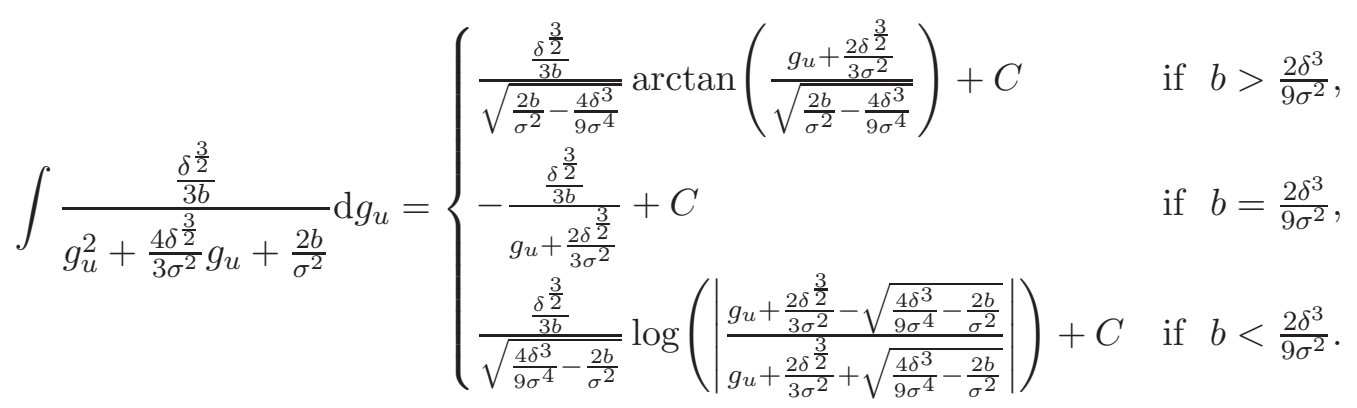

Consequently, if $b>\frac{2 \delta^{3}}{9 \sigma^{2}}$, then

$$
-\frac{\sigma^{2}}{4 b} \log \left(1+\frac{4 \delta^{\frac{3}{2}}}{3 \sigma^{2} g_{u}(t)}+\frac{2 b}{\sigma^{2} g_{u}(t)^{2}}\right)-\frac{\frac{\delta^{\frac{3}{2}}}{3 b}}{\sqrt{\frac{2 b}{\sigma^{2}}-\frac{4 \delta^{3}}{9 \sigma^{4}}}} \arctan \left(\frac{g_{u}(t)+\frac{2 \delta^{\frac{3}{2}}}{3 \sigma^{2}}}{\sqrt{\frac{2 b}{\sigma^{2}}-\frac{4 \delta^{3}}{9 \sigma^{4}}}}\right)=-\frac{\sigma^{2}}{4} t+C, \quad t \in \mathbb{R}_{+},
$$

with some $C \in \mathbb{R}_{+}$. Using the initial value $g_{u}(0)=(-u)^{\frac{1}{2}}$, we obtain

$$
C=-\frac{\sigma^{2}}{4 b} \log \left(1+\frac{4 \delta^{\frac{3}{2}}}{3 \sigma^{2}(-u)^{\frac{1}{2}}}-\frac{2 b}{\sigma^{2} u}\right)-\frac{\frac{\delta^{\frac{3}{2}}}{3 b}}{\sqrt{\frac{2 b}{\sigma^{2}}-\frac{4 \delta^{3}}{9 \sigma^{4}}}} \arctan \left(\frac{(-u)^{\frac{1}{2}}+\frac{2 \delta^{\frac{3}{2}}}{3 \sigma^{2}}}{\sqrt{\frac{2 b}{\sigma^{2}}-\frac{4 \delta^{3}}{9 \sigma^{4}}}}\right),
$$

and hence, by $g_{u}(t)=\left(-\psi_{u, 0}(t)\right)^{\frac{1}{2}}$, we conclude

$$
\begin{aligned}
& \frac{\sigma^{2}}{4 b} \log \left(\frac{1+\frac{4 \delta^{\frac{3}{2}}}{3 \sigma^{2}\left(-\psi_{u, 0}(t)\right)^{\frac{1}{2}}}-\frac{2 b}{\sigma^{2} \psi_{u, 0}(t)}}{1+\frac{4 \delta^{\frac{3}{2}}}{3 \sigma^{2}(-u)^{\frac{1}{2}}}-\frac{2 b}{\sigma^{2} u}}\right) \\
& +\frac{\frac{\delta^{\frac{3}{2}}}{3 b}}{\sqrt{\frac{2 b}{\sigma^{2}}-\frac{4 \delta^{3}}{9 \sigma^{4}}}}\left(\arctan \left(\frac{\left(-\psi_{u, 0}(t)\right)^{\frac{1}{2}}+\frac{2 \delta^{\frac{3}{2}}}{3 \sigma^{2}}}{\sqrt{\frac{2 b}{\sigma^{2}}-\frac{4 \delta^{3}}{9 \sigma^{4}}}}\right)-\arctan \left(\frac{(-u)^{\frac{1}{2}}+\frac{2 \delta^{\frac{3}{2}}}{3 \sigma^{2}}}{\sqrt{\frac{2 b}{\sigma^{2}}-\frac{4 \delta^{3}}{9 \sigma^{4}}}}\right)\right)=\frac{\sigma^{2}}{4} t .
\end{aligned}
$$

In a similar way, if $b=\frac{2 \delta^{3}}{9 \sigma^{2}}$, then

$$
-\frac{\sigma^{2}}{4 b} \log \left(1+\frac{4 \delta^{\frac{3}{2}}}{3 \sigma^{2} g_{u}(t)}+\frac{2 b}{\sigma^{2} g_{u}(t)^{2}}\right)+\frac{\frac{\delta^{\frac{3}{2}}}{3 b}}{g_{u}(t)+\frac{2 \delta^{\frac{3}{2}}}{3 \sigma^{2}}}=-\frac{\sigma^{2}}{4} t+C, \quad t \in \mathbb{R}_{+},
$$

with some $C \in \mathbb{R}_{+}$. Using the initial value $g_{u}(0)=(-u)^{\frac{1}{2}}$, we obtain

$$
C=-\frac{\sigma^{2}}{4 b} \log \left(1+\frac{4 \delta^{\frac{3}{2}}}{3 \sigma^{2}(-u)^{\frac{1}{2}}}-\frac{2 b}{\sigma^{2} u}\right)+\frac{\frac{\delta^{\frac{3}{2}}}{3 b}}{(-u)^{\frac{1}{2}}+\frac{2 \delta^{\frac{3}{2}}}{3 \sigma^{2}}}
$$


and hence, by $g_{u}(t)=\left(-\psi_{u, 0}(t)\right)^{\frac{1}{2}}$, we conclude

$$
\frac{\sigma^{2}}{4 b} \log \left(\frac{1+\frac{4 \delta^{\frac{3}{2}}}{3 \sigma^{2}\left(-\psi_{u, 0}(t)\right)^{\frac{1}{2}}}-\frac{2 b}{\sigma^{2} \psi_{u, 0}(t)}}{1+\frac{4 \delta^{\frac{3}{2}}}{3 \sigma^{2}(-u)^{\frac{1}{2}}}-\frac{2 b}{\sigma^{2} u}}\right)-\frac{\frac{\delta^{\frac{3}{2}}}{3 b}}{\left(-\psi_{u, 0}(t)\right)^{\frac{1}{2}}+\frac{2 \delta^{\frac{3}{2}}}{3 \sigma^{2}}}+\frac{\frac{\delta^{\frac{3}{2}}}{3 b}}{(-u)^{\frac{1}{2}}+\frac{2 \delta^{\frac{3}{2}}}{3 \sigma^{2}}}=\frac{\sigma^{2}}{4} t .
$$

Further, if $b \neq 0$ and $b<\frac{2 \delta^{3}}{9 \sigma^{2}}$, then

$$
\begin{aligned}
& \frac{\sigma^{2}}{2 b} \log \left(g_{u}(t)\right)-\frac{\sigma^{2}}{4 b} \log \left(\left|g_{u}(t)^{2}+\frac{4 \delta^{\frac{3}{2}}}{3 \sigma^{2}} g_{u}(t)+\frac{2 b}{\sigma^{2}}\right|\right) \\
& -\frac{\frac{\delta^{\frac{3}{2}}}{3 b}}{\sqrt{\frac{4 \delta^{3}}{9 \sigma^{4}}-\frac{2 b}{\sigma^{2}}}} \log \left(\left|\frac{g_{u}(t)+\frac{2 \delta^{\frac{3}{2}}}{3 \sigma^{2}}-\sqrt{\frac{4 \delta^{3}}{9 \sigma^{4}}-\frac{2 b}{\sigma^{2}}}}{g_{u}(t)+\frac{2 \delta^{\frac{3}{2}}}{3 \sigma^{2}}+\sqrt{\frac{4 \delta^{3}}{9 \sigma^{4}}-\frac{2 b}{\sigma^{2}}}}\right|\right)=-\frac{\sigma^{2}}{4} t+C, \quad t \in \mathbb{R}_{+},
\end{aligned}
$$

with some $C \in \mathbb{R}$. Using the initial value $g_{u}(0)=(-u)^{\frac{1}{2}}$, we obtain

$$
\begin{aligned}
C= & \frac{\sigma^{2}}{2 b} \log \left((-u)^{\frac{1}{2}}\right)-\frac{\sigma^{2}}{4 b} \log \left(\left|-u+\frac{4 \delta^{\frac{3}{2}}}{3 \sigma^{2}}(-u)^{\frac{1}{2}}+\frac{2 b}{\sigma^{2}}\right|\right) \\
& -\frac{\frac{\delta^{\frac{3}{2}}}{3 b}}{\sqrt{\frac{4 \delta^{3}}{9 \sigma^{4}}-\frac{2 b}{\sigma^{2}}}} \log \left(\left|\frac{(-u)^{\frac{1}{2}}+\frac{2 \delta^{\frac{3}{2}}}{3 \sigma^{2}}-\sqrt{\frac{4 \delta^{3}}{9 \sigma^{4}}-\frac{2 b}{\sigma^{2}}}}{(-u)^{\frac{1}{2}}+\frac{2 \delta^{\frac{3}{2}}}{3 \sigma^{2}}+\sqrt{\frac{4 \delta^{3}}{9 \sigma^{4}}-\frac{2 b}{\sigma^{2}}}}\right|\right),
\end{aligned}
$$

and hence, by $g_{u}(t)=\left(-\psi_{u, 0}(t)\right)^{\frac{1}{2}}$, we conclude

$$
\begin{aligned}
& -\frac{\sigma^{2}}{2 b} \log \left(\left(-\psi_{u, 0}(t)\right)^{\frac{1}{2}}\right)+\frac{\sigma^{2}}{4 b} \log \left(\left|-\psi_{u, 0}(t)+\frac{4 \delta^{\frac{3}{2}}}{3 \sigma^{2}}\left(-\psi_{u, 0}(t)\right)^{\frac{1}{2}}+\frac{2 b}{\sigma^{2}}\right|\right) \\
& +\frac{\sigma^{2}}{2 b} \log \left((-u)^{\frac{1}{2}}\right)-\frac{\sigma^{2}}{4 b} \log \left(\left|-u+\frac{4 \delta^{\frac{3}{2}}}{3 \sigma^{2}}(-u)^{\frac{1}{2}}+\frac{2 b}{\sigma^{2}}\right|\right) \\
& +\frac{\frac{\delta^{\frac{3}{2}}}{3 b}}{\sqrt{\frac{4 \delta^{3}}{9 \sigma^{4}}-\frac{2 b}{\sigma^{2}}}} \log \left(\left|\frac{\left(-\psi_{u, 0}(t)\right)^{\frac{1}{2}}+\frac{2 \delta^{\frac{3}{2}}}{3 \sigma^{2}}-\sqrt{\frac{4 \delta^{3}}{9 \sigma^{4}}-\frac{2 b}{\sigma^{2}}}}{\left(-\psi_{u, 0}(t)\right)^{\frac{1}{2}}+\frac{2 \delta^{\frac{3}{2}}}{3 \sigma^{2}}+\sqrt{\frac{4 \delta^{3}}{9 \sigma^{4}}-\frac{2 b}{\sigma^{2}}}}\right|\right) \\
& -\frac{\frac{\delta^{\frac{3}{2}}}{3 b}}{\sqrt{\frac{4 \delta^{3}}{9 \sigma^{4}}-\frac{2 b}{\sigma^{2}}}} \log \left(\left|\frac{(-u)^{\frac{1}{2}}+\frac{2 \delta^{\frac{3}{2}}}{3 \sigma^{2}}-\sqrt{\frac{4 \delta^{3}}{9 \sigma^{4}}-\frac{2 b}{\sigma^{2}}}}{(-u)^{\frac{1}{2}}+\frac{2 \delta^{\frac{3}{2}}}{3 \sigma^{2}}+\sqrt{\frac{4 \delta^{3}}{9 \sigma^{4}}-\frac{2 b}{\sigma^{2}}}}\right|\right)=\frac{\sigma^{2}}{4} t .
\end{aligned}
$$

Finally, if $b=0$, then, by separation of variables, we have

$$
\frac{1}{\left(g_{u}+\frac{4 \delta^{\frac{3}{2}}}{3 \sigma^{2}}\right) g_{u}^{2}} \mathrm{~d} g_{u}=-\frac{\sigma^{2}}{4} \mathrm{~d} t
$$

where

$$
\frac{1}{\left(g_{u}+\frac{4 \delta^{\frac{3}{2}}}{3 \sigma^{2}}\right) g_{u}^{2}}=\frac{\frac{9 \sigma^{4}}{16 \delta^{3}}}{g_{u}+\frac{4 \delta^{\frac{3}{2}}}{3 \sigma^{2}}}-\frac{\frac{9 \sigma^{4}}{16 \delta^{3}}}{g_{u}}+\frac{3 \sigma^{2}}{4 \delta^{\frac{3}{2}} g_{u}^{2}}
$$


hence

$$
\begin{aligned}
\int \frac{1}{\left(g_{u}+\frac{4 \delta^{\frac{3}{2}}}{3 \sigma^{2}}\right) g_{u}^{2}} \mathrm{~d} g_{u} & =\frac{9 \sigma^{4}}{16 \delta^{3}} \log \left(g_{u}+\frac{4 \delta^{\frac{3}{2}}}{3 \sigma^{2}}\right)-\frac{9 \sigma^{4}}{16 \delta^{3}} \log \left(g_{u}\right)-\frac{3 \sigma^{2}}{4 \delta^{\frac{3}{2}} g_{u}}+C \\
& =\frac{9 \sigma^{4}}{16 \delta^{3}} \log \left(1+\frac{4 \delta^{\frac{3}{2}}}{3 \sigma^{2} g_{u}}\right)-\frac{3 \sigma^{2}}{4 \delta^{\frac{3}{2}} g_{u}}+C
\end{aligned}
$$

yielding

$$
\frac{9 \sigma^{4}}{16 \delta^{3}} \log \left(1+\frac{4 \delta^{\frac{3}{2}}}{3 \sigma^{2} g_{u}(t)}\right)-\frac{3 \sigma^{2}}{4 \delta^{\frac{3}{2}} g_{u}(t)}=-\frac{\sigma^{2}}{4} t+C, \quad t \in \mathbb{R}_{+},
$$

with some $C \in \mathbb{R}$. Using the initial value $g_{u}(0)=(-u)^{\frac{1}{2}}$, we obtain

$$
C=\frac{9 \sigma^{4}}{16 \delta^{3}} \log \left(1+\frac{4 \delta^{\frac{3}{2}}}{3 \sigma^{2}(-u)^{\frac{1}{2}}}\right)-\frac{3 \sigma^{2}}{4 \delta^{\frac{3}{2}}(-u)^{\frac{1}{2}}},
$$

and hence, by $g_{u}(t)=\left(-\psi_{u, 0}(t)\right)^{\frac{1}{2}}$, we conclude

$$
\frac{9 \sigma^{4}}{16 \delta^{3}} \log \left(\frac{1+\frac{4 \delta^{\frac{3}{2}}}{3 \sigma^{2}\left(-\psi_{u, 0}(t)\right)^{\frac{1}{2}}}}{1+\frac{4 \delta^{\frac{3}{2}}}{3 \sigma^{2}(-u)^{\frac{1}{2}}}}\right)-\frac{3 \sigma^{2}}{4 \delta^{\frac{3}{2}}}\left(\frac{1}{\left(-\psi_{u, 0}(t)\right)^{\frac{1}{2}}}-\frac{1}{(-u)^{\frac{1}{2}}}\right)=-\frac{\sigma^{2}}{4} t .
$$

C.3 Example. We derive an explicit formula for the Laplace transform of $V$ given in Theorem 7.1 in case of $\alpha=\frac{3}{2}$. In fact, we present two detailed calculations, the first one is based on the representation of $\psi_{u}^{*}$ given in part (iii) of Theorem [7.1, and the second one is based on part (ii) of Theorem 7.1 .

Calculations based on part (iii) of Theorem 7.1. We have

$$
\mathbb{E}\left(\mathrm{e}^{u V}\right)=\exp \left\{y_{0} \psi_{u}^{*}+a \int_{0}^{-\psi_{u}^{*}} \frac{1}{\frac{\sigma^{2}}{2} x+\frac{2 \delta^{\frac{3}{2}}}{3} x^{\frac{1}{2}}+b} \mathrm{~d} x\right\}, \quad u \in \mathbb{R}_{-},
$$

with $\psi_{0}^{*}=0$ and $\psi_{u}^{*}=-K^{-1}(-u)$ for $u \in \mathbb{R}_{--}$, where $K^{-1}$ is the inverse of the strictly increasing function $K:\left(0, \theta_{0}\right) \rightarrow \mathbb{R}_{++}$given by

$$
K(\lambda)=\lambda \exp \left\{-\int_{0}^{\lambda} \frac{\frac{\sigma^{2}}{2}+\frac{2 \delta^{\frac{3}{2}}}{3} x^{-\frac{1}{2}}}{\frac{\sigma^{2}}{2} x+\frac{2 \delta^{\frac{3}{2}}}{3} x^{\frac{1}{2}}+b} \mathrm{~d} x\right\}, \quad \lambda \in\left(0, \theta_{0}\right),
$$

where we used (7.2) and

$$
\theta_{0}=\inf \left\{x \in \mathbb{R}_{++}: \frac{\sigma^{2}}{2} x^{2}+\frac{2 \delta^{\frac{3}{2}}}{3} x^{\frac{3}{2}}+b x \in \mathbb{R}_{+}\right\}=\left(-\frac{2 \delta^{\frac{3}{2}}}{3 \sigma^{2}}+\sqrt{\frac{4 \delta^{3}}{9 \sigma^{4}}-\frac{2 b}{\sigma^{2}}}\right)^{2} .
$$

By substitution $x=y^{2}$, for all $\lambda \in\left(0, \theta_{0}\right)$, we have

$$
\int_{0}^{\lambda} \frac{\frac{\sigma^{2}}{2}+\frac{2 \delta^{\frac{3}{2}}}{3} x^{-\frac{1}{2}}}{\frac{\sigma^{2}}{2} x+\frac{2 \delta^{\frac{3}{2}}}{3} x^{\frac{1}{2}}+b} \mathrm{~d} x=\int_{0}^{\lambda^{\frac{1}{2}}} \frac{\sigma^{2} y+\frac{4 \delta^{\frac{3}{2}}}{3}}{\frac{\sigma^{2}}{2} y^{2}+\frac{2 \delta^{\frac{3}{2}}}{3} y+b} \mathrm{~d} y .
$$


First we consider the case of $\sigma \in \mathbb{R}_{++}$. Then we can write

$$
\frac{\sigma^{2} x+\frac{4 \delta^{\frac{3}{2}}}{3}}{\frac{\sigma^{2}}{2} x^{2}+\frac{2 \delta^{\frac{3}{2}}}{3} x+b}=\frac{2 x+\frac{4 \delta^{\frac{3}{2}}}{3 \sigma^{2}}}{x^{2}+\frac{4 \delta^{\frac{3}{2}}}{3 \sigma^{2}} x+\frac{2 b}{\sigma^{2}}}+\frac{4 \delta^{\frac{3}{2}}}{3 \sigma^{2}} \cdot \frac{1}{x^{2}+\frac{4 \delta^{\frac{3}{2}}}{3 \sigma^{2}} x+\frac{2 b}{\sigma^{2}}} .
$$

As in Example C.1, we have

$$
\int_{0}^{\lambda^{\frac{1}{2}}} \frac{2 x+\frac{4 \delta^{\frac{3}{2}}}{3 \sigma^{2}}}{x^{2}+\frac{4 \delta^{\frac{3}{2}}}{3 \sigma^{2}} x+\frac{2 b}{\sigma^{2}}} \mathrm{~d} x=\log \left(\frac{\sigma^{2}}{2 b} \lambda+\frac{2 \delta^{\frac{3}{2}}}{3 b} \lambda^{\frac{1}{2}}+1\right)
$$

and

$$
\int_{0}^{\lambda^{\frac{1}{2}}} \frac{1}{x^{2}+\frac{4 \delta^{\frac{3}{2}}}{3 \sigma^{2}} x+\frac{2 b}{\sigma^{2}}} \mathrm{~d} x=\frac{1}{\sqrt{\frac{4 \delta^{3}}{9 \sigma^{4}}-\frac{2 b}{\sigma^{2}}}} \log \left(\frac{\lambda^{\frac{1}{2}}\left(\frac{2 \delta^{\frac{3}{2}}}{3 \sigma^{2}}+\sqrt{\frac{4 \delta^{3}}{9 \sigma^{4}}-\frac{2 b}{\sigma^{2}}}\right)+\frac{2 b}{\sigma^{2}}}{\lambda^{\frac{1}{2}}\left(\frac{2 \delta^{\frac{3}{2}}}{3 \sigma^{2}}-\sqrt{\frac{4 \delta^{3}}{9 \sigma^{4}}-\frac{2 b}{\sigma^{2}}}\right)+\frac{2 b}{\sigma^{2}}}\right)
$$

and hence

$$
\begin{aligned}
K(\lambda) & =\frac{\lambda}{\frac{\sigma^{2}}{2 b} \lambda+\frac{2 \delta^{\frac{3}{2}}}{3 b} \lambda^{\frac{1}{2}}+1}\left(\frac{\lambda^{\frac{1}{2}}\left(\frac{2 \delta^{\frac{3}{2}}}{3 \sigma^{2}}+\sqrt{\frac{4 \delta^{3}}{9 \sigma^{4}}-\frac{2 b}{\sigma^{2}}}\right)+\frac{2 b}{\sigma^{2}}}{\lambda^{\frac{1}{2}}\left(\frac{2 \delta^{\frac{3}{2}}}{3 \sigma^{2}}-\sqrt{\frac{4 \delta^{3}}{9 \sigma^{4}}-\frac{2 b}{\sigma^{2}}}\right)+\frac{2 b}{\sigma^{2}}}\right)^{-\frac{\frac{4 \delta^{\frac{3}{2}}}{3 \sigma^{2}}}{\sqrt{\frac{4 \delta^{3}}{9 \sigma^{4}}-\frac{2 b}{\sigma^{2}}}}} \\
& =\left(\frac{\delta^{\frac{3}{2}}}{3 b}+\sqrt{\frac{\delta^{3}}{9 b^{2}}-\frac{\sigma^{2}}{2 b}}+\lambda^{-\frac{1}{2}}\right)^{-1-\frac{\frac{4 \delta^{\frac{3}{2}}}{3 \sigma^{2}}}{\sqrt{\frac{4 \delta^{3}}{9 \sigma^{4}}-\frac{2 b}{\sigma^{2}}}}}\left(\frac{\delta^{\frac{3}{2}}}{3 b}-\sqrt{\frac{\delta^{3}}{9 b^{2}}-\frac{\sigma^{2}}{2 b}}+\lambda^{-\frac{1}{2}}\right)^{-1+\frac{\frac{4 \delta^{\frac{3}{2}}}{3 \sigma^{2}}}{\sqrt{\frac{4 \delta^{3}}{9 \sigma^{4}}-\frac{2 b}{\sigma^{2}}}}} .
\end{aligned}
$$

Note that an explicit formula for $K^{-1}$ is not available. Next we consider the case of $\sigma=0$. By Remark 7.3, we have $\theta_{0}=\frac{9 b^{2}}{4 \delta^{3}}$,

$$
K(\lambda)=\left(\frac{2 \delta^{\frac{3}{2}}}{3 b}+\lambda^{-\frac{1}{2}}\right)^{-2}, \quad \lambda \in\left(0, \frac{9 b^{2}}{4 \delta^{3}}\right),
$$

thus

$$
\psi_{u}^{*}=-K^{-1}(-u)=-\left((-u)^{-\frac{1}{2}}-\frac{2 \delta^{\frac{3}{2}}}{3 b}\right)^{-2},
$$

and hence

$$
\mathbb{E}\left(\mathrm{e}^{u V}\right)=\exp \left\{-y_{0}\left((-u)^{-\frac{1}{2}}-\frac{2 \delta^{\frac{3}{2}}}{3 b}\right)^{-2}+a \int_{0}^{\left((-u)^{-\frac{1}{2}}-\frac{2 \delta^{\frac{3}{2}}}{3 b}\right)^{-2}} \frac{1}{\frac{2 \delta^{\frac{3}{2}}}{3} x^{\frac{1}{2}}+b} \mathrm{~d} x\right\}, \quad u \in \mathbb{R}_{--} .
$$

By substitution $x=y^{2}$, for all $u \in \mathbb{R}_{--}$, we have

$$
\int_{0}^{\left((-u)^{-\frac{1}{2}}-\frac{2 \delta^{\frac{3}{2}}}{3 b}\right)^{-2}} \frac{1}{\frac{2 \delta^{\frac{3}{2}}}{3} x^{\frac{1}{2}}+b} \mathrm{~d} x=\int_{0}^{\left((-u)^{-\frac{1}{2}}-\frac{2 \delta^{\frac{3}{2}}}{3 b}\right)^{-1}} \frac{2 y}{\frac{2 \delta^{\frac{3}{2}}}{3} y+b} \mathrm{~d} y .
$$

As in Example C.1, we can write

$$
\frac{2 y}{\frac{2 \delta^{\frac{3}{2}}}{3} y+b}=\frac{3}{\delta^{\frac{3}{2}}}-\frac{\frac{9 b}{2 \delta^{3}}}{y+\frac{3 b}{2 \delta^{\frac{3}{2}}}},
$$


thus

$$
\begin{aligned}
& \int_{0}^{\left((-u)^{-\frac{1}{2}}-\frac{2 \delta^{\frac{3}{2}}}{3 b}\right)^{-1}} \frac{2 y}{\frac{2 \delta^{\frac{3}{2}}}{3} y+b} \mathrm{~d} y=\frac{3}{\delta^{\frac{3}{2}}}\left((-u)^{-\frac{1}{2}}-\frac{2 \delta^{\frac{3}{2}}}{3 b}\right)^{-1} \\
& -\frac{9 b}{2 \delta^{3}}\left(\log \left(\left|\left((-u)^{-\frac{1}{2}}-\frac{2 \delta^{\frac{3}{2}}}{3 b}\right)^{-1}+\frac{3 b}{2 \delta^{\frac{3}{2}}}\right|\right)-\log \left(\left|\frac{3 b}{2 \delta^{\frac{3}{2}}}\right|\right)\right) \\
& =\frac{3}{\delta^{\frac{3}{2}}}\left((-u)^{-\frac{1}{2}}-\frac{2 \delta^{\frac{3}{2}}}{3 b}\right)^{-1}+\frac{9 b}{2 \delta^{3}} \log \left(1-\frac{2 \delta^{\frac{3}{2}}}{3 b}(-u)^{\frac{1}{2}}\right),
\end{aligned}
$$

and hence

$$
\mathbb{E}\left(\mathrm{e}^{u V}\right)=\exp \left\{-y_{0}\left((-u)^{-\frac{1}{2}}-\frac{2 \delta^{\frac{3}{2}}}{3 b}\right)^{-2}\right\} \exp \left\{\frac{3 a}{\delta^{\frac{3}{2}}}\left((-u)^{-\frac{1}{2}}-\frac{2 \delta^{\frac{3}{2}}}{3 b}\right)^{-1}\right\}\left(1-\frac{2 \delta^{\frac{3}{2}}}{3 b}(-u)^{\frac{1}{2}}\right)^{\frac{9 b a}{2 \delta^{3}}}
$$

for $u \in \mathbb{R}_{--}$.

Calculations based on part (ii) of Theorem 7.1. By (7.1), it is enough to know $\psi_{u}^{*}$ to have an explicit formula for the Laplace transform of $V$. We carry out this calculation only in case of $\sigma \in \mathbb{R}_{++}$. By (C.6), for all $t \in \mathbb{R}_{+}$and $u \in \mathbb{R}_{--}$with $u \mathrm{e}^{b t} \neq u_{0}$, we obtain

$$
\begin{aligned}
& -\frac{\sigma^{2}}{2 b} \log \left(\left(-\psi_{u \mathrm{e}^{b t}, 0}(t)\right)^{\frac{1}{2}}\right)+\frac{\sigma^{2}}{4 b} \log \left(\left|-\psi_{u \mathrm{e}^{b t}, 0}(t)+\frac{4 \delta^{\frac{3}{2}}}{3 \sigma^{2}}\left(-\psi_{u \mathrm{e}^{b t}, 0}(t)\right)^{\frac{1}{2}}+\frac{2 b}{\sigma^{2}}\right|\right) \\
& +\frac{\sigma^{2}}{2 b} \log \left(\left(-u \mathrm{e}^{b t}\right)^{\frac{1}{2}}\right)-\frac{\sigma^{2}}{4 b} \log \left(\left|-u \mathrm{e}^{b t}+\frac{4 \delta^{\frac{3}{2}}}{3 \sigma^{2}}\left(-u \mathrm{e}^{b t}\right)^{\frac{1}{2}}+\frac{2 b}{\sigma^{2}}\right|\right) \\
& +\frac{\frac{\delta^{\frac{3}{2}}}{3 b}}{\sqrt{\frac{4 \delta^{3}}{9 \sigma^{4}}-\frac{2 b}{\sigma^{2}}}} \log \left(\left|\frac{\left(-\psi_{u \mathrm{e}^{b t}, 0}(t)\right)^{\frac{1}{2}}+\frac{2 \delta^{\frac{3}{2}}}{3 \sigma^{2}}-\sqrt{\frac{4 \delta^{3}}{9 \sigma^{4}}-\frac{2 b}{\sigma^{2}}}}{\left(-\psi_{u \mathrm{e}^{b t}, 0}(t)\right)^{\frac{1}{2}}+\frac{2 \delta^{\frac{3}{2}}}{3 \sigma^{2}}+\sqrt{\frac{4 \delta^{3}}{9 \sigma^{4}}-\frac{2 b}{\sigma^{2}}}}\right|\right) \\
& -\frac{\frac{\delta^{\frac{3}{2}}}{3 b}}{\sqrt{\frac{4 \delta^{3}}{9 \sigma^{4}}-\frac{2 b}{\sigma^{2}}}} \log \left(\left|\frac{\left(-u \mathrm{e}^{b t}\right)^{\frac{1}{2}}+\frac{2 \delta^{\frac{3}{2}}}{3 \sigma^{2}}-\sqrt{\frac{4 \delta^{3}}{9 \sigma^{4}}-\frac{2 b}{\sigma^{2}}}}{\left(-u \mathrm{e}^{b t}\right)^{\frac{1}{2}}+\frac{2 \delta^{\frac{3}{2}}}{3 \sigma^{2}}+\sqrt{\frac{4 \delta^{3}}{9 \sigma^{4}}-\frac{2 b}{\sigma^{2}}}}\right|\right)=\frac{\sigma^{2}}{4} t .
\end{aligned}
$$

Using $\frac{\sigma^{2}}{2 b} \log \left(\left(-u \mathrm{e}^{b t}\right)^{\frac{1}{2}}\right)=\frac{\sigma^{2}}{4 b} \log (-u)+\frac{\sigma^{2}}{4} t$, we conclude that $\psi_{u}^{*}$ satisfies the equation

$$
\begin{aligned}
& -\frac{\sigma^{2}}{4} \log \left(-\psi_{u}^{*}\right)+\frac{\sigma^{2}}{4} \log \left(\left|-\psi_{u}^{*}+\frac{4 \delta^{\frac{3}{2}}}{3 \sigma^{2}}\left(-\psi_{u}^{*}\right)^{\frac{1}{2}}+\frac{2 b}{\sigma^{2}}\right|\right)+\frac{\sigma^{2}}{4} \log (-u)-\frac{\sigma^{2}}{4} \log \left(-\frac{2 b}{\sigma^{2}}\right) \\
& +\frac{\frac{\delta^{\frac{3}{2}}}{3}}{\sqrt{\frac{4 \delta^{3}}{9 \sigma^{4}}-\frac{2 b}{\sigma^{2}}}}\left(\log \left(\left|\frac{\left(-\psi_{u}^{*}\right)^{\frac{1}{2}}+\frac{2 \delta^{\frac{3}{2}}}{3 \sigma^{2}}-\sqrt{\frac{4 \delta^{3}}{9 \sigma^{4}}-\frac{2 b}{\sigma^{2}}}}{\left(-\psi_{u}^{*}\right)^{\frac{1}{2}}+\frac{2 \delta^{\frac{3}{2}}}{3 \sigma^{2}}+\sqrt{\frac{4 \delta^{3}}{9 \sigma^{4}}-\frac{2 b}{\sigma^{2}}}}\right|\right)-\log \left(\frac{\sqrt{\frac{4 \delta^{3}}{9 \sigma^{4}}-\frac{2 b}{\sigma^{2}}}-\frac{2 \delta^{\frac{3}{2}}}{3 \sigma^{2}}}{\sqrt{\frac{4 \delta^{3}}{9 \sigma^{4}}-\frac{2 b}{\sigma^{2}}}+\frac{2 \delta^{\frac{3}{2}}}{3 \sigma^{2}}}\right)\right)=0 .
\end{aligned}
$$

By Theorem 7.1, $R\left(-\psi_{u}^{*}\right)<0$. We have $R\left(-\psi_{u}^{*}\right)=\frac{\sigma^{2}}{2}\left(-\psi_{u}^{*}+\frac{4 \delta^{\frac{3}{2}}}{3 \sigma^{2}}\left(-\psi_{u}^{*}\right)^{\frac{1}{2}}+\frac{2 b}{\sigma^{2}}\right)\left(-\psi_{u}^{*}\right)$, where $-\psi_{u}^{*} \in\left(0, \theta_{0}\right)$, hence $-\psi_{u}^{*}+\frac{4 \delta^{\frac{3}{2}}}{3 \sigma^{2}}\left(-\psi_{u}^{*}\right)^{\frac{1}{2}}+\frac{2 b}{\sigma^{2}}<0$. Moreover,

$$
-\psi_{u}^{*}+\frac{4 \delta^{\frac{3}{2}}}{3 \sigma^{2}}\left(-\psi_{u}^{*}\right)^{\frac{1}{2}}+\frac{2 b}{\sigma^{2}}=\left(\left(-\psi_{u}^{*}\right)^{\frac{1}{2}}+\frac{2 \delta^{\frac{3}{2}}}{3 \sigma^{2}}-\sqrt{\frac{4 \delta^{3}}{9 \sigma^{4}}-\frac{2 b}{\sigma^{2}}}\right)\left(\left(-\psi_{u}^{*}\right)^{\frac{1}{2}}+\frac{2 \delta^{\frac{3}{2}}}{3 \sigma^{2}}+\sqrt{\frac{4 \delta^{3}}{9 \sigma^{4}}-\frac{2 b}{\sigma^{2}}}\right),
$$


where $\left(-\psi_{u}^{*}\right)^{\frac{1}{2}}+\frac{2 \delta^{\frac{3}{2}}}{3 \sigma^{2}}+\sqrt{\frac{4 \delta^{3}}{9 \sigma^{4}}-\frac{2 b}{\sigma^{2}}}>0$, thus $\left(-\psi_{u}^{*}\right)^{\frac{1}{2}}+\frac{2 \delta^{\frac{3}{2}}}{3 \sigma^{2}}-\sqrt{\frac{4 \delta^{3}}{9 \sigma^{4}}-\frac{2 b}{\sigma^{2}}}<0$. Consequently, $\psi_{u}^{*}$ satisfies the equation

$$
\begin{aligned}
& -\frac{\sigma^{2}}{4} \log \left(-\psi_{u}^{*}\right)+\frac{\sigma^{2}}{4} \log \left(\psi_{u}^{*}-\frac{4 \delta^{\frac{3}{2}}}{3 \sigma^{2}}\left(-\psi_{u}^{*}\right)^{\frac{1}{2}}-\frac{2 b}{\sigma^{2}}\right)+\frac{\sigma^{2}}{4} \log (-u)-\frac{\sigma^{2}}{4} \log \left(-\frac{2 b}{\sigma^{2}}\right) \\
& +\frac{\frac{\delta^{\frac{3}{2}}}{3}}{\sqrt{\frac{4 \delta^{3}}{9 \sigma^{4}}-\frac{2 b}{\sigma^{2}}}}\left(\log \left(\frac{\sqrt{\frac{4 \delta^{3}}{9 \sigma^{4}}-\frac{2 b}{\sigma^{2}}}-\left(-\psi_{u}^{*}\right)^{\frac{1}{2}}-\frac{2 \delta^{\frac{3}{2}}}{3 \sigma^{2}}}{\sqrt{\frac{4 \delta^{3}}{9 \sigma^{4}}-\frac{2 b}{\sigma^{2}}}+\left(-\psi_{u}^{*}\right)^{\frac{1}{2}}+\frac{2 \delta^{\frac{3}{2}}}{3 \sigma^{2}}}\right)-\log \left(\frac{\sqrt{\frac{4 \delta^{3}}{9 \sigma^{4}}-\frac{2 b}{\sigma^{2}}}-\frac{2 \delta^{\frac{3}{2}}}{3 \sigma^{2}}}{\sqrt{\frac{4 \delta^{3}}{9 \sigma^{4}}-\frac{2 b}{\sigma^{2}}}+\frac{2 \delta^{\frac{3}{2}}}{3 \sigma^{2}}}\right)\right)=0 .
\end{aligned}
$$

Note that, by Theorem 7.1, (C.8) is equivalent to $K\left(-\psi_{u}^{*}\right)=-u$.

We show another way to derive this equation. By Theorem $\mathbf{7 . 1}$, for all sufficiently small $\lambda \in \mathbb{R}_{++}$,

$$
\begin{aligned}
& \int_{\lambda}^{-\psi_{u}^{*}} \frac{1}{\frac{\sigma^{2}}{2} z^{2}+\frac{2 \delta^{\frac{3}{2}}}{3} z^{\frac{3}{2}}+b z} \mathrm{~d} z=\int_{\lambda}^{f\left(\frac{-u}{K(\lambda)}, \lambda\right)} \frac{1}{R(z)} \mathrm{d} z=\frac{1}{b} \log \left(-\frac{u}{K(\lambda)}\right) \\
& =\frac{1}{b} \log (-u)-\frac{1}{b} \log (K(\lambda))=\frac{1}{b} \log (-u)-\frac{1}{b} \log (\lambda)-\frac{1}{b} \int_{0}^{\lambda}\left(\frac{b}{R(z)}-\frac{1}{z}\right) \mathrm{d} z .
\end{aligned}
$$

By substitution $z=y^{2}$, and using (C.5),

$$
\begin{aligned}
& \int_{\lambda}^{-\psi_{u}^{*}} \frac{1}{\frac{\sigma^{2}}{2} z^{2}+\frac{2 \delta^{\frac{3}{2}}}{3} z^{\frac{3}{2}}+b z} \mathrm{~d} z=\int_{\sqrt{\lambda}}^{\left(-\psi_{u}^{*}\right)^{\frac{1}{2}}} \frac{2}{\frac{\sigma^{2}}{2} y^{3}+\frac{2 \delta^{\frac{3}{2}}}{3} y^{2}+b y} \mathrm{~d} y \\
& =\left[\frac{2}{b} \log (|y|)-\frac{1}{b} \log \left(\left|y^{2}+\frac{4 \delta^{\frac{3}{2}}}{3 \sigma^{2}} y+\frac{2 b}{\sigma^{2}}\right|\right)-\frac{\frac{4 \delta^{\frac{3}{2}}}{3 \sigma^{2} b}}{\sqrt{\frac{4 \delta^{3}}{9 \sigma^{4}}-\frac{2 b}{\sigma^{2}}}} \log \left(\left|\frac{y+\frac{2 \delta^{\frac{3}{2}}}{3 \sigma^{2}}-\sqrt{\frac{4 \delta^{3}}{9 \sigma^{4}}-\frac{2 b}{\sigma^{2}}}}{y+\frac{2 \delta^{\frac{3}{2}}}{3 \sigma^{2}}+\sqrt{\frac{4 \delta^{3}}{9 \sigma^{4}}-\frac{2 b}{\sigma^{2}}}}\right|\right)\right]_{y=\sqrt{\lambda}}^{y=\left(-\psi_{u}^{*}\right)^{\frac{1}{2}}} \\
& =\frac{1}{b} \log \left(-\psi_{u}^{*}\right)-\frac{1}{b} \log (\lambda)-\frac{1}{b} \log \left(\left|-\psi_{u}^{*}+\frac{4 \delta^{\frac{3}{2}}}{3 \sigma^{2}}\left(-\psi_{u}^{*}\right)^{\frac{1}{2}}+\frac{2 b}{\sigma^{2}}\right|\right)+\frac{1}{b} \log \left(\left|\lambda+\frac{4 \delta^{\frac{3}{2}}}{3 \sigma^{2}} \sqrt{\lambda}+\frac{2 b}{\sigma^{2}}\right|\right) \\
& -\frac{\frac{4 \delta^{\frac{3}{2}}}{3 \sigma^{2} b}}{\sqrt{\frac{4 \delta^{3}}{9 \sigma^{4}}-\frac{2 b}{\sigma^{2}}}}\left(\log \left(\left|\frac{\left(-\psi_{u}^{*}\right)^{\frac{1}{2}}+\frac{2 \delta^{\frac{3}{2}}}{3 \sigma^{2}}-\sqrt{\frac{4 \delta^{3}}{9 \sigma^{4}}-\frac{2 b}{\sigma^{2}}}}{\left(-\psi_{u}^{*}\right)^{\frac{1}{2}}+\frac{2 \delta^{\frac{3}{2}}}{3 \sigma^{2}}+\sqrt{\frac{4 \delta^{3}}{9 \sigma^{4}}-\frac{2 b}{\sigma^{2}}}}\right|\right)-\log \left(\left|\frac{\sqrt{\lambda}+\frac{2 \delta^{\frac{3}{2}}}{3 \sigma^{2}}-\sqrt{\frac{4 \delta^{3}}{9 \sigma^{4}}-\frac{2 b}{\sigma^{2}}}}{\sqrt{\lambda}+\frac{2 \delta^{\frac{3}{2}}}{3 \sigma^{2}}+\sqrt{\frac{4 \delta^{3}}{9 \sigma^{4}}-\frac{2 b}{\sigma^{2}}}}\right|\right)\right) .
\end{aligned}
$$

Moreover,

$$
\int_{0}^{\lambda}\left(\frac{b}{R(z)}-\frac{1}{z}\right) \mathrm{d} z=\int_{0}^{\lambda}\left(\frac{b}{\frac{\sigma^{2}}{2} z^{2}+\frac{2 \delta^{\frac{3}{2}}}{3} z^{\frac{3}{2}}+b z}-\frac{1}{z}\right) \mathrm{d} z=-\int_{0}^{\lambda} \frac{\frac{\sigma^{2}}{2}+\frac{2 \delta^{\frac{3}{2}}}{3} z^{-\frac{1}{2}}}{\frac{\sigma^{2}}{2} z+\frac{2 \delta^{\frac{3}{2}}}{3} z^{\frac{1}{2}}+b} \mathrm{~d} z .
$$

By substitution $z=y^{2}$,

$$
\int_{0}^{\lambda} \frac{\frac{\sigma^{2}}{2}+\frac{2 \delta^{\frac{3}{2}}}{3} z^{-\frac{1}{2}}}{\frac{\sigma^{2}}{2} z+\frac{2 \delta^{\frac{3}{2}}}{3} z^{\frac{1}{2}}+b} \mathrm{~d} z=\int_{0}^{\sqrt{\lambda}} \frac{\sigma^{2} y+\frac{4 \delta^{\frac{3}{2}}}{3}}{\frac{\sigma^{2}}{2} y^{2}+\frac{2 \delta^{\frac{3}{2}}}{3} y+b} \mathrm{~d} y .
$$

We can write

$$
\frac{\sigma^{2} y+\frac{4 \delta^{\frac{3}{2}}}{3}}{\frac{\sigma^{2}}{2} y^{2}+\frac{2 \delta^{\frac{3}{2}}}{3} y+b}=\frac{\sigma^{2} y+\frac{2 \delta^{\frac{3}{2}}}{3}}{\frac{\sigma^{2}}{2} y^{2}+\frac{2 \delta^{\frac{3}{2}}}{3} y+b}+\frac{\frac{2 \delta^{\frac{3}{2}}}{3}}{\frac{\sigma^{2}}{2} y^{2}+\frac{2 \delta^{\frac{3}{2}}}{3} y+b}
$$


hence, by (C.4),

$$
\begin{aligned}
& \int_{0}^{\lambda}\left(\frac{b}{R(z)}-\frac{1}{z}\right) \mathrm{d} z=-\int_{0}^{\sqrt{\lambda}} \frac{\sigma^{2} y+\frac{4 \delta^{\frac{3}{2}}}{3}}{\frac{\sigma^{2}}{2} y^{2}+\frac{2 \delta^{\frac{3}{2}}}{3} y+b} \mathrm{~d} y \\
& =-\left[\log \left(\left|\frac{\sigma^{2}}{2} y^{2}+\frac{2 \delta^{\frac{3}{2}}}{3} y+b\right|\right)+\frac{\frac{4 \delta^{\frac{3}{2}}}{3 \sigma^{2}}}{\sqrt{\frac{4 \delta^{3}}{9 \sigma^{4}}-\frac{2 b}{\sigma^{2}}}} \log \left(\left|\frac{y+\frac{2 \delta^{\frac{3}{2}}}{3 \sigma^{2}}-\sqrt{\frac{4 \delta^{3}}{9 \sigma^{4}}-\frac{2 b}{\sigma^{2}}}}{y+\frac{2 \delta^{\frac{3}{2}}}{3 \sigma^{2}}+\sqrt{\frac{4 \delta^{3}}{9 \sigma^{4}}-\frac{2 b}{\sigma^{2}}}}\right|\right)\right]_{y=0}^{y=\sqrt{\lambda}} \\
& =-\log \left(\left|\frac{\sigma^{2}}{2} \lambda+\frac{2 \delta^{\frac{3}{2}}}{3} \sqrt{\lambda}+b\right|\right)+\log (-b) \\
& -\frac{\frac{4 \delta^{\frac{3}{2}}}{3 \sigma^{2}}}{\sqrt{\frac{4 \delta^{3}}{9 \sigma^{4}}-\frac{2 b}{\sigma^{2}}}}\left(\log \left(\left|\frac{\sqrt{\lambda}+\frac{2 \delta^{\frac{3}{2}}}{3 \sigma^{2}}-\sqrt{\frac{4 \delta^{3}}{9 \sigma^{4}}-\frac{2 b}{\sigma^{2}}}}{\sqrt{\lambda}+\frac{2 \delta^{\frac{3}{2}}}{3 \sigma^{2}}+\sqrt{\frac{4 \delta^{3}}{9 \sigma^{4}}-\frac{2 b}{\sigma^{2}}}}\right|\right)-\log \left(\frac{\sqrt{\frac{4 \delta^{3}}{9 \sigma^{4}}-\frac{2 b}{\sigma^{2}}}-\frac{2 \delta^{\frac{3}{2}}}{3 \sigma^{2}}}{\sqrt{\frac{4 \delta^{3}}{9 \sigma^{4}}-\frac{2 b}{\sigma^{2}}}+\frac{2 \delta^{\frac{3}{2}}}{3 \sigma^{2}}}\right)\right)
\end{aligned}
$$

Consequently, (C.9) yields again that $\psi_{u}^{*}$ satisfies equation (C.7), and hence, equation (C.8).

\section{Acknowledgements}

We are grateful to Clément Foucart for providing us an idea how to derive (17.1), a formula for the Laplace transform of $V$ in Theorem 7.1. We would like to thank Hatem Zaag for explaining us several methods that may be used for describing the asymptotic behavior of the ordinary differential equation (3.1). We would like to thank the referees for their comments that helped us to improve the paper.

\section{References}

[1] Amann, H. (1990). Ordinary Differential Equations. An Introduction to Nonlinear Analysis. Walter de Gruyter, Berlin, New York.

[2] Barczy, M., Ben Alaya, M., Kebaier, A. and Pap, G. (2019). Asymptotic behavior of maximum likelihood estimators for a jump-type Heston model. Journal of Statistical Planning and Inference 198 139-164.

[3] Arnol'D, V. I. (1992). Ordinary differential equations Springer-Verlag, Berlin.

[4] Barczy, M., Döring, L., Li, Z. and Pap, G. (2014). Stationarity and ergodicity for an affine two factor model. Advances in Applied Probability 46(3) 878-898.

[5] Barczy, M., Li, Z. and PAP, G. (2015). Stochastic differential equation with jumps for multitype continuous state and continuous time branching processes with immigration. ALEA. Latin American Journal of Probability and Mathematical Statistics. 12(1) 129-169.

[6] Barczy, M. and PAP, G. (2016). Asymptotic properties of maximum-likelihood estimators for Heston models based on continuous time observations. Statistics. 50(2) 389-417. 
[7] Billingsley, P. (1999). Convergence of probability measures, 2nd ed. John Wiley \& Sons, Inc., New York.

[8] Bhattacharya, R. N. (1982). On the functional central limit theorem and the law of the iterated logarithm for Markov processes. Zeitschrift für Wahrscheinlichkeitstheorie und Verwandte Gebiete $60185-201$.

[9] Carr, P. and Wu, L. (2004). Time-changed Lévy processes and option pricing. Journal of Financial Economics 71 113-141.

[10] Dawson, D. A. and Li, Z. (2006). Skew convolution semigroups and affine Markov processes. The Annals of Probability 34(3) 1103-1142.

[11] Dudley, R. M. (1989). Real Analysis and Probability. Wadsworth \& Brooks/Cole Advanced Books \& Software, Pacific Grove, California.

[12] Duffie, D., Filipović, D. and Schachermayer, W. (2003). Affine processes and applications in finance. Annals of Applied Probability 13(3) 984-1053.

[13] Filipović, D. (2001). A general characterization of one factor affine term structure models. Finance and Stochastics 5(3) 389-412.

[14] Filipović, D., Mayerhofer, E. and Schneider, P. (2013). Density approximations for multivariate affine jump-diffusion processes. Journal of Econometrics 176(2) 93-111.

[15] Fu, Z. and LI, Z. (2010). Stochastic equations of non-negative processes with jumps. Stochastic Processes and their Applications 120 306-330.

[16] JACOD, J. and MÉmin, J. (1976). Caractéristiques locales et conditions de continuité absolue pour les semi-martingales Zeitschrift für Wahrscheinlichkeitstheorie und Verwandte Gebiete, 35 $1-37$.

[17] Jacod, J. and Protter, P. (2012). Discretization of processes, Springer, Heidelberg.

[18] Jacod, J. and Shinyaev, A. N. (2003). Limit Theorems for Stochastic Processes, 2nd ed. Springer-Verlag, Berlin.

[19] Jiao, Y., Ma, C. and Scotti, S. (2017). Alpha-CIR model with branching processes in sovereign interest rate modelling. Finance and Stochastics 21(3) 789-813.

[20] JiaO, Y., Ma, C., Scotti, S. and Sgarra, C. (2018+). A branching process approach to power markets. To appear in Energy Economics. DOI https://doi.org/10.1016/j.eneco.2018.03.002

[21] Jin, P., Kremer, J. and Rüdiger, B. (2017). Exponential ergodicity of an affine two-factor model based on the $\alpha$-root process. Advances in Applied Probability 49(4) 1144-1169.

[22] Keller-Ressel, M. (2008). Affine Processes - Theory and Applications in Finance. PhD Thesis, Vienna University of Technology, pages 110.

[23] Keller-Ressel, M. (2011). Moment explosions and long-term behavior of affine stochastic volatility models. Mathematical Finance 21(1) 73-98. 
[24] Küchler, U. and Sørensen, M. (1997). Exponential families of stochastic processes, SpringerVerlag, New York.

[25] LI, Z. (2011). Measure-Valued Branching Markov Processes. Springer-Verlag, Heidelberg.

[26] Li, Z. and MA, C. (2015). Asymptotic properties of estimators in a stable Cox-Ingersoll-Ross model. Stochastic Processes and their Applications 125(8) 3196-3233.

[27] LI, Z. (2012). Continuous-state branching processes. Available on ArXiv: http://arxiv.org/abs/1202.3223

[28] Liptser, R. S. and Shiryaev, A. N. (2001). Statistics of Random Processes II. Applications, 2nd edition. Springer-Verlag, Berlin, Heidelberg.

[29] Luschgy, H. (1992). Local asymptotic mixed normality for semimartingale experiments. Probability Theory and Related Fields 92 151-176.

[30] Luschay, H. (1994). Asymptotic inference for semimartingale models with singular parameter points. Journal of Statistical Planning and Inference 39 155-186.

[31] MA, R. and YAng, X. (2014). Small noise fluctuations of the CIR model driven by $\alpha$-stable noises. Statistics and Probability Letters 94 1-11.

[32] Overbeck, L. (1998). Estimation for continuous branching processes. Scandinavian Journal of Statistics 25(1) 111-126.

[33] Peng, J. (2016). A stable Cox-Ingersoll-Ross model with restart. Journal of Mathematical Analysis and Applications 444 1185-1194.

[34] Polyanin, A. D. and Zaitsev, V. F. (2003). Handbook of Exact Solutions for Ordinary Differential Equations. Chapman \& Hall/CRC, Boca Raton London New York Washington, D.C.

[35] Sato, K.-I. (1999). Lévy Processes and Infinitely Divisible Distributions. Cambridge University Press, Cambridge.

[36] Sørensen, M. (1991). Likelihood methods for diffusions with jumps. In: N. U. Prabhu and I. V. Basawa, Eds., Statistical Inference in Stochastic Processes, Marcel Dekker, New York, 67-105.

[37] VAn Zanten, H. (2000). A multivariate central limit theorem for continuous local martingales. Statistics \&6 Probability Letters 50(3) 229-235.

[38] YAnG, X. (2017). Maximum likelihood type estimation for discretely observed CIR model with small $\alpha$-stable noises. Statistics $\&$ Probability Letters 120 18-27. 